\title{
Charakterisierung und Modifizierung glatter laserdeponierter Poly(methyl methacrylat)-Schichten
}

\author{
Dissertation \\ zur Erlangung des mathematisch-naturwissenschaftlichen \\ Doktorgrades \\ „Doctor rerum naturalium“ \\ der Georg-August-Universität Göttingen
}

vorgelegt von

Britta Fuchs

geb. Lösekrug

aus Peine

Göttingen, 2010 
D7

Referent:

Prof. Dr. H.-U. Krebs

Korreferent:

Prof. Dr. M. Buback

Tag der mündlichen Prüfung: 23. Februar 2010 
Meinem persönlichen Admin 


\section{Inhaltsverzeichnis}

$\begin{array}{lll}1 & \text { Einleitung } & 1\end{array}$

2 Experimentelle Methoden 5

2.1 Herstellung der Schichten. . . . . . . . . . . . . . . . . . . . 5

2.1.1 Gepulste Laserdeposition (PLD) . . . . . . . . . . . . . . . . 5

2.1.2 $\quad$ Targets und Substrate $\ldots \ldots \ldots$. . . . . . . . . . . 7

$2.1 .3 \quad$ Nachbehandlung der Schichten. . . . . . . . . . . . . . . . . . . . 8

2.2 Analyse der Probenmorphologie . . . . . . . . . . . . . . . . . . . . . 9

$2.2 .1 \quad$ Rasterelektronenmikroskopie (SEM) . . . . . . . . . . . . . . . 9 9

2.2 .2 Rasterkraftmikroskopie $(\mathrm{AFM}) \quad \ldots \ldots$. . . . . . . . . . . . . 9

$2.2 .3 \quad$ Auflichtmikroskopie . . . . . . . . . . . . . . . . . . . . . . . . . . . . . 9

2.2 .4 Röntgenreflektometrie (XRR) . . . . . . . . . . . . . . . . . . . . . . . . . . . . . 10

2.2 .5 Ellipsometrie $\ldots \ldots \ldots \ldots \ldots$

2.2 .6 Profilometrie . . . . . . . . . . . . . . . . . . . 11

2.3 Materialanalyse . . . . . . . . . . . . . . . . . . . . . 11

2.3.1 Fouriertransformations-IR-Spektroskopie (FT-IR) . . . . . . . 11

2.3.2 Röntgennahkantenabsorptionsspektroskopie (NEXAFS) . . . . 13

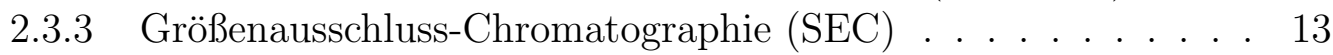

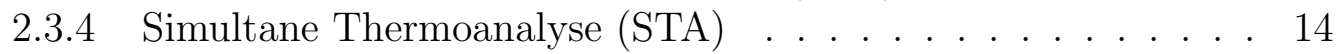

2.3.5 Massenspektrometrie . . . . . . . . . . . . . . . 14

2.4 Mechanische Charakterisierungsmethoden . . . . . . . . . . . . . . 15

$2.4 .1 \quad$ Ultramikrohärteprüfung . . . . . . . . . . . . . . . . . . 15

3 Ablation und Deposition 17

3.1 Poly(methyl methacrylat) (PMMA) . . . . . . . . . . . . . . . . . . 17

3.2 Einfluss der Laserfluenz . . . . . . . . . . . . . . . . . . . . . . . . . 20

3.3 Morphologie . . . . . . . . . . . . . . . . . . . . . . 25

3.4 Schichtdickenverteilung . . . . . . . . . . . . . . . . . . . . . . . . . 29

3.5 Chemische Eigenschaften . . . . . . . . . . . . . . . . . . . . . . . . 31

4 Charakterisierung glatter PMMA-Schichten 33

4.1 Inkubation . . . . . . . . . . . . . . . . . 33

4.2 Schichtdickenabhängigkeit . . . . . . . . . . . . . . . . . . . . . . . 36

4.3 Schichtdickenverteilung . . . . . . . . . . . . . . . . . . . . . . . . . . 39 
4.4 Chemische Eigenschaften . . . . . . . . . . . . . . . . . . . . . . . . . 41

4.5 Mechanische Eigenschaften . . . . . . . . . . . . . . . . . . . . . . 44

4.6 Fazit . . . . . . . . . . . . . . . . . . . . . . . . . . . . . 48

\begin{tabular}{|lll}
5 & Thermische Behandlung der PMMA-Schichten & 49
\end{tabular}

5.1 Bestimmung der Glasübergangstemperatur . . . . . . . . . . . . . . . 49

5.2 Thermische Stabilität . . . . . . . . . . . . . . . . . . . . . 52

5.3 Chemische Eigenschaften . . . . . . . . . . . . . . . . . . . 53

5.4 Analyse der Schichtfragmente . . . . . . . . . . . . . . . . . 56

5.5 Fazit . . . . . . . . . . . . . . . . . . . . . 64

6 Modifizierung glatter PMMA-Schichten $\quad 67$

6.1 Modifizierung durch UV-Bestrahlung der Schichten . . . . . . . . . . 67

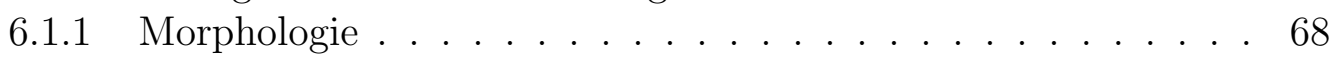

6.1 .2 Chemische Eigenschaften . . . . . . . . . . . . . . . . . . . . . 69

$6.1 .3 \quad$ Mechanische Eigenschaften . . . . . . . . . . . . . . . . . . 71

6.2 Modifizierung durch zusätzlichen Vernetzer im Target . . . . . . . . . 73

$6.2 .1 \quad$ Morphologie . . . . . . . . . . . . . . . . 75

$6.2 .2 \quad$ Chemische Eigenschaften . . . . . . . . . . . . . . . . . . . . 77

$6.2 .3 \quad$ Mechanische Eigenschaften . . . . . . . . . . . . . . . . . . . . 79

6.3 Modifizierung durch thermische Behandlung . . . . . . . . . . . . . . 80

6.3 .1 Morphologie . . . . . . . . . . . . . . . . . 80

6.3 .2 Mechanische Eigenschaften . . . . . . . . . . . . . . . . . 83

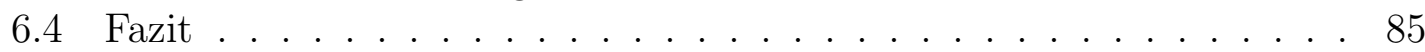

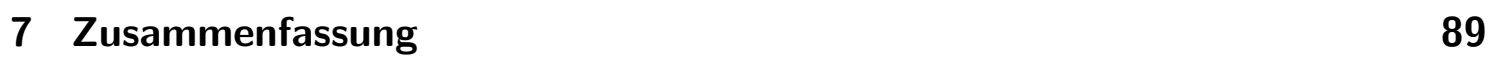

\begin{tabular}{ll}
\hline Literaturverzeichnis & 93
\end{tabular} 


\section{Einleitung}

Heutzutage werden Polymere in sehr vielen Lebensbereichen erfolgreich eingesetzt. So setzen sie sich gegen andere Materialien aufgrund vieler Vorteile immer mehr durch. Zu den Vorzügen der Polymere zählen dabei besonders ihre geringe Dichte, ihre hohe Korrosionsbeständigkeit, ihre mechanischen Eigenschaften wie Bruchfestigkeit und Zähigkeit oder auch ihre einfache Verarbeitung, wodurch das Produkt meist sehr kostengünstig ist. In der Anwendung als Werkstoff sind die synthetischen Polymere führend, deren Produktionsmenge sogar das Volumen von Stahl als klassischen Werkstoff erreicht hat [48. Abhängig von dem Anwendungsgebiet der Polymere z. B. als Verpackungsmaterial und für Textilien oder für optische Anwendungen oder in elektronischen Bauteilen werden ihre benötigten Eigenschaften festgelegt. Diese werden maßgeblich durch ihre Struktur bestimmt, die sich durch lange Hauptketten mit Seitengruppen, die in sich wiederholende Monomereinheiten unterteilt werden, durch ihr Molekulargewicht und durch den Grad der Vernetzung der Ketten untereinander auszeichnet.

Vor allem in den letzten Jahren wurde für viele der Anwendungsgebiete eine Verarbeitung der Polymere als dünne Schichten immer bedeutender. Dies betrifft z. B. die weiterentwickelte Miniaturisierung im Bereich der Mikroelektronik, sowie optische, katalytische, mikrosensorische und pharmazeutische Anwendungen [15].

Ebenso interessant wie einkomponentige Polymere sind dabei auch Kompositmaterialien, die durch die Verbindung verschiedener Materialien die Möglichkeit bieten, die positiven Eigenschaften mehrerer Werkstoffe zu vereinen [50]. Besondere Anforderungen werden vor allem dann an die Herstellungsmethode gestellt, wenn es um die Vereinigung verschiedener Materialklassen geht, da diese oft sehr unterschiedliche, teilweise inkompatible Prozesse erfordern.

Für solche komplexen Anforderungen sind Standardverfahren wie das Aufspinnen (spin coating) als nasschemisches Verfahren meist nicht geeignet, da es zu Problemen bzw. Unverträglichkeiten mit dem dabei verwendeten Lösungsmittel kommen kann. Für Polymere, bei denen diese Probleme nicht auftreten, lässt sich mit diesem Verfahren aber eine gut reproduzierbare chemische Struktur der polymeren Schicht erreichen [28]. Um Unverträglichkeiten mit den verwendeten Lösungsmitteln auf jeden Fall zu entgehen, kann auch das Verfahren der Plasmapolymerisation verwendet werden [88]. Diese kann aber widerum nur für wenige Polymere angewendet werden und produziert chemisch stark vernetzte Polymere. Andere industriell wichtige Verfahren zur Herstellung von Polymerschichten sind z. B. auch das Aufdampfen [73] oder die elektrochemische Polymerisation [85]. 
Als eine sehr flexible Methode zur Herstellung dünner Schichten vieler Materialklassen bietet sich aber die gepulste Laserdeposition (pulsed laser deposition, PLD) an [14, 23]. Entwickelt wurde diese Methode 1965 von SMith und TuRner 91. Anfangs wurde die PLD vornehmlich für die Herstellung von HochtemperaturSupraleitern verwendet [20], bis dann auch Halbleiter und Keramiken oder Metalle [52] deponiert wurden. Das Interesse an der Herstellung von laserdeponierten Polymerschichten nimmt seit einiger Zeit ebenfalls immer mehr zu. Erste laserdeponierte Polymerschichten - darunter auch Schichten aus Poly(methyl methacrylat) (PMMA) - wurden Ende der achtziger Jahre von HANsen und Robitaille untersucht [45.

Das Ausgangsmaterial Poly(methyl methacrylat) (PMMA) ist ein transparenter, amorpher Thermoplast, der erstmals 1933 unter dem Namen Plexiglas ${ }^{\circledR}$ von Отто RöHM vermarktet wurde. Angewendet wird PMMA inzwischen z. B. als Kontaktlinsen, in der Zahnmedizin für Prothesen und Provisorien, in der Medizin als Knochenzement zur Stabilisierung von Implantaten im Knochen (z. B. zur Verankerung von Hüftendoprothesen) und in der Photolithographie als Photolack.

Die Laserdeposition von PMMA unter Verwendung eines KrF-Excimerlasers mit einer Wellenlänge von $248 \mathrm{~nm}$ wurde bisher z. B. in den Arbeiten von SüskE [104] und ScharF [87] näher untersucht. Dabei entstanden allerdings durch die Laserdeposition zweikomponentige Schichten, von denen die eine Komponente einen glatten Schichtanteil aus hauptsächlich hochvernetztem PMMA und die andere Komponente eine hohe Anzahl von PMMA-Tröpfchen (droplets) mit einem Durchmesser von einigen $\mu \mathrm{m}$ darstellte. Diese Morphologie der PMMA-Schichten stellt sich bedingt durch den niedrigen Absorptionskoeffizienten des Materials bei der verwendeten Wellenlänge ein [44, 45]. Die Schichteigenschaften hängen aber nicht allein von der eingestrahlten Wellenlänge [17] oder der Pulsdauer [57] des verwendeten Lasers ab, sondern ändern sich auch stark mit der Bestrahlungsintensität. So war es in der vorangegangenen Diplomarbeit durch eine starke Absenkung der verwendeten Laserfluenz erstmals möglich, glatte Poly(methyl methacrylat)-Schichten mit der gepulsten Laserdeposition herzustellen [70]. Diese Schichten bestehen nur noch aus einer sehr glatten Komponente und sind absolut tröpfchenfrei [69]. Durch die geringe Rauigkeit der auf diese Weise deponierten Schichten wird die praktische Anwendung nun sehr viel interessanter.

Ausgehend von diesen ersten Untersuchungen der glatten PMMA-Schichten sollen in dieser Arbeit weiterführende Experimente zur Charakterisierung der Schichteigenschaften diskutiert werden. Daraus sollen Erkenntnisse über die durch die PLD angeworfenen Prozesse gewonnen werden, die zur Bildung dieser Schichten mit ihren charakteristischen Eigenschaften führen. Dabei spielt z. B. der der Ablation vorangehende Inkubationsprozess am Ausgangsmaterial eine wichtige Rolle. Neben den Untersuchungen zur Schichtmorphologie sollen vor allem chemische Analysen und mechanische Untersuchungen dazu beitragen, die zugrundeliegenden Mechanismen zu erklären. Insbesondere geht es dabei um die schon länger andauernde, kontroverse Diskussion in der Literatur, ob es sich bei der Laserdeposition von Polymeren 
vorwiegend um einen photochemischen oder um einen photothermischen Ablationsmechanismus handelt [97, 1, 56]. Inzwischen wird angenommen, dass es sich bei einer Bestrahlung mit einer Wellenlänge von $248 \mathrm{~nm}$ eher um einen zweistufigen Prozess handelt, bei dem beide Mechanismen nacheinander ablaufen [22, 15]. Anwendungsbezogen sind auch thermische Untersuchungen hinsichtlich der Stabilität und des Einflusses auf weitere charakteristische Eigenschaften laserdeponierter PMMASchichten gemacht worden. Da sich vor allem durch thermische Behandlungen in den mechanischen Eigenschaften - in Form der Härte - große Änderungen einstellten, wurden hier als einer der Schwerpunkte drei Möglichkeiten zur Beeinflussung der Universalhärte untersucht und diese Änderungen der mechanischen Eigenschaften zusammen mit den entsprechenden Änderungen der Morphologie und der chemischen Eigenschaften diskutiert.

Die Arbeit gliedert sich damit wie folgt: Zunächst soll in Kapitel 22 die Vielzahl der verwendeten experimentellen Methoden vorgestellt werden. Dafür wird zunächst die Methode und der verwendete Aufbau der gepulsten Laserdeposition zur Herstellung der PMMA-Schichten sowie die Möglichkeiten der nachträglichen Behandlung der Schichten vorgestellt. Dann werden kurz die verwendeten Targets und Substrate beschrieben, bevor sich die verschiedenen Charakterisierungsmethoden anschließen. Diese wurden unterteilt in Methoden zur Analysierung der Oberflächenmorphologie, der chemischen Zusammensetzung sowie der mechanischen Eigenschaften, was die Messung der Universalhärte beinhaltet.

In Kapitel 3 wird eine Zusammenfassung der Ergebnisse gegeben, die aus der vorangegangenen Diplomarbeit über die glatten PMMA-Schichten resultieren. Dabei wurde vor allem der Einfluss der verwendeten Laserfluenz auf die Schichtmorphologie untersucht und damit der Bereich der Laserfluenz - kurz oberhalb der Depositionsschwelle - gefunden, in dem eine Herstellung glatter Schichten überhaupt erst möglich ist.

Daran schließt sich in Kapitel 4 die weitere Charakterisierung dieser glatt deponierten PMMA-Schichten an, wobei nun neben den Morphologieuntersuchungen besonders auf den für die PLD von PMMA sehr wichtigen Inkubationsprozess eingegangen wird. Außerdem lassen sich die bei der Schichtdeposition ablaufenden Prozesse durch weitere chemische Untersuchungen sowie durch eine Analyse der Härteeigenschaften gut diskutieren.

In Kapitel 5 werden Untersuchung in Bezug auf die thermische Stabilität der PMMA-Schichten durchgeführt. Hierzu wird zunächst die Glasübergangstemperatur des PMMA vor und nach der Deposition vergleichend diskutiert. Durch Messung der dabei entstehenden Schichtfragmente lassen sich außerdem Schlüsse bezüglich des thermisch aktivierten Anteils der Schichtdeposition ziehen. Weitere chemische Änderungen im PMMA-Schichtmaterial, die durch den thermischen Einfluss stattfinden, werden beschrieben und das Maß der thermischen Zersetzung bei hohen Temperaturen wird diskutiert.

Aufgrund der zuvor gewonnenen Erkenntnisse soll in Kapitel 6 untersucht wer- 
den, inwiefern sich die glatten PMMA-Schichten besonders in Bezug auf ihre mechanischen Eigenschaften modifizieren lassen. Durch eine Erhöhung der Härtewerte werden praktische Anwendungen der deponierten Schichten verbessert. In dieser Arbeit werden drei verschiedene Möglichkeiten der Modifizierung vorgestellt und die Ergebnisse sowohl der mechanischen Untersuchungen als auch der Morphologiebetrachtungen und der chemischen Untersuchungen miteinander verglichen. Dabei handelt es sich z. B. um eine nachträgliche Bestrahlung der Schichten mit ultraviolettem Licht. Dies ist eine gängige Methode der Polymervernetzung in Bezug auf PMMA als Photolack in der Lithographie. Eine andere Vorgehensweise ist die Mischung des PMMA-Ausgangsmaterials mit einer vernetzenden Komponente bevor aus dieser Mischung die verwendeten Targets gepresst werden. Außerdem werden an dieser Stelle die Morphologie- und Härteänderungen vorgestellt, wie sie durch die im vorangegangenen Kapitel beschriebenen thermischen Behandlungen induziert werden.

Abschließend werden in Kapitel 7 die hiesigen Ergebnisse zusammengefasst. 


\section{Experimentelle Methoden}

Zunächst sollen die im Rahmen dieser Arbeit verwendeten experimentellen Methoden vorgestellt werden. Angefangen mit der gepulsten Laserdeposition von Polymerschichten wird dann näher auf die Modifikation der Schichten eingegangen und anschließend werden die verwendeten Charakterisierungsmethoden beschrieben.

\subsection{Herstellung der Schichten}

Nach der Beschreibung der Depositionsmethode selbst wird auch auf die verwendeten Targets und Substrate und auf die Möglichkeiten der zusätzlichen Beeinflussung der Schichteigenschaften eingegangen.

\subsubsection{Gepulste Laserdeposition (PLD)}

Die gepulste Laserdeposition (Pulsed Laser Deposition, PLD) hat sich von den Anfängen in den sechziger Jahren von SMITH und TuRner [91] bis heute zu einer wichtigen Methode zur Herstellung dünner Schichten aller erdenklichen Materialien entwickelt [14, 23]. Hierbei wurde die PLD anfangs vornehmlich für die Herstellung von Hochtemperatur-Supraleitern verwendet [20], bis dann auch Halbleiter und Keramiken oder Metalle [52] deponiert wurden. Das Interesse an der Herstellung von laserdeponierten Polymerschichten nimmt seit einiger Zeit ebenfalls immer mehr zu. Andere industriell wichtige Verfahren zur Herstellung von Polymerschichten sind z. B. Aufdampfen [73], Plasma-Polymerisation [88, elektrochemische Polymerisation [85] oder Aufspinnen [28]. Erste laserdeponierte Polymerschichten wurden erst Ende der achtziger Jahre von Hansen und RoBitaille untersucht [45].

Für die Herstellung der Schichten wurde ein typischer Aufbau verwendet [53], wie er in Abb. 2.1 schematisch zu sehen ist. Es wurde ein gepulster Excimerlaser LPX 110i der Firma LAmBDA PHYSIK mit einem Krypton-Fluor-Gasgemisch verwendet. Das angeregte KrF-Dimer emittiert bei jedem Übergang in den Grundzustand ein Photon mit einer Energie von $5 \mathrm{eV}$, was einer Wellenlänge von $248 \mathrm{~nm}$ entspricht. Der Laser erzeugt auf diese Weise ultraviolette (UV) Pulse mit einer Pulsdauer von $30 \mathrm{~ns}$ ( full width at half maximum, FWHM) und einer maximalen Energie von 300 mJ. Die bei der Schichtherstellung verwendeten Fluenzen werden später diskutiert. Um ein zu großes Aufheizen des Polymers während der Herstellung zu vermeiden, wird eine 


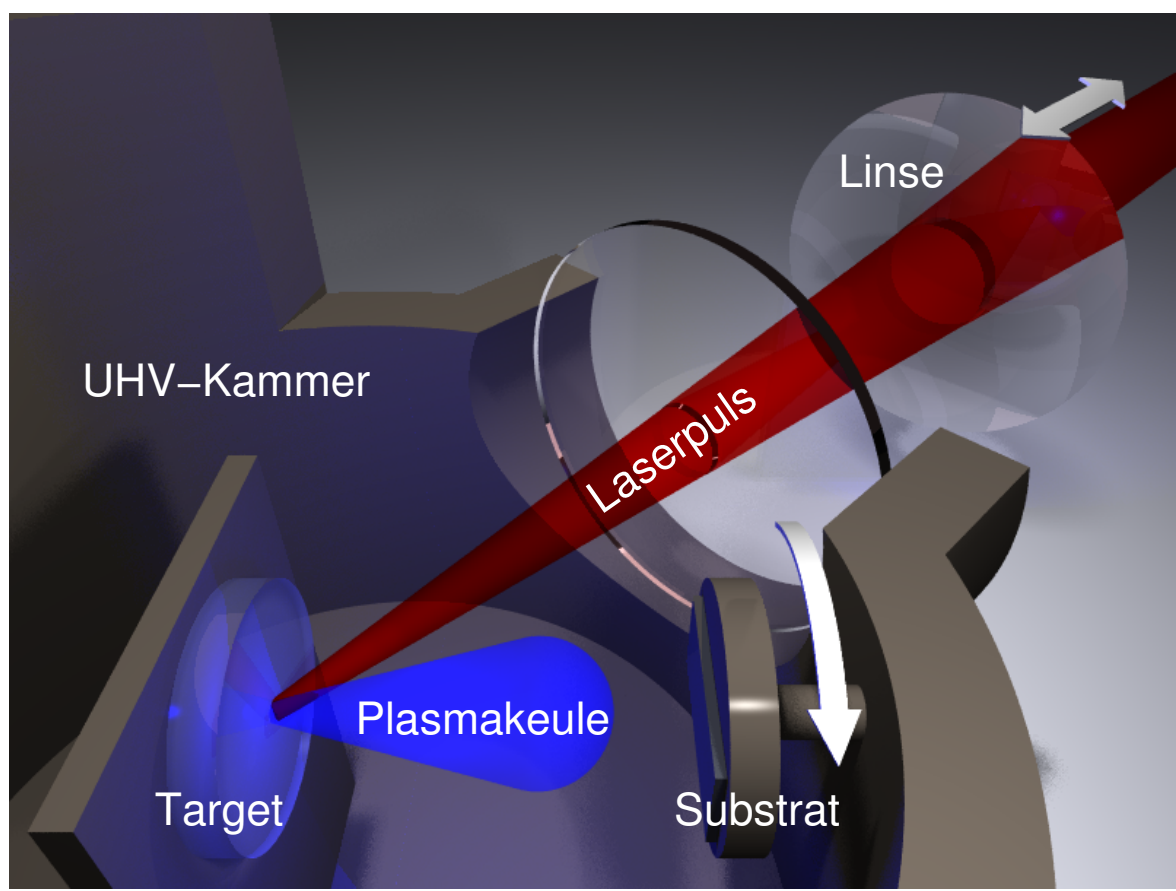

Abbildung 2.1: Schematischer Aufbau der verwendeten PLD-Kammer. Die Laserpulse treffen unter $45^{\circ}$ mit $\lambda=248 \mathrm{~nm}$ und $\tau=30 \mathrm{~ns}$ auf die Targetoberfläche. Das rotierende Substrat befindet sich mit einem Abstand von $5 \mathrm{~cm}$ gegenüber der Targetoberfläche.

Repetitionsrate von nur $5 \mathrm{~Hz}$ gewählt [58]. Der Laserstrahl tritt durch ein Fenster in die Ultrahochvakuumkammer. Diese wird für die Schichtdeposition durch ein UHV-Pumpsystem, bestehend aus einer Rotations- und einer Turbomolekularpumpe, auf einen Druck im Bereich von $10^{-8}$ mbar evakuiert. Damit ist der Einfluss des Restgasdrucks auf den Depositionsprozess zu vernachlässigen.

Der Laserstrahl trifft in der Kammer ein eingebautes Bulkmaterial (Target) unter einem Winkel von $45^{\circ}$. Die Größe des Brennflecks auf der Targetoberfläche wird durch die Position einer fokussierenden Linse im Strahlengang außerhalb der Kammer eingestellt. Die Ausdehnung des Brennflecks lässt sich so von $0,017 \mathrm{~cm}^{2}$ bis $2,5 \mathrm{~cm}^{2}$ variieren. Damit und mit der Variation der Hochspannung des Lasers von $18 \mathrm{kV}$ bis $26 \mathrm{kV}$ und mit einem zusätzlichen halbdurchlässigen Spiegel als Abschwächer im Strahlengang lässt sich ein sehr großer Energiedichtebereich von $20 \mathrm{~mJ} \mathrm{~cm}^{-2}$ bis zu $9000 \mathrm{~mJ} \mathrm{~cm}^{-2}$ abdecken [70].

Die Ausdehnung des Brennflecks am Target ist in der Einfallsebene des Laserstrahls (horizontal) um das Doppelte bis Dreifache größer als seine senkrechte (vertikal) Ausdehnung. Durch eine höhere Ausdehnung in horizontaler Richtung ist die Stoßwahrscheinlichkeit einzelner Atome oder Moleküle in dieser Richtung höher, wodurch die mittlere Geschwindigkeit der Teilchen stärker abnimmt als in vertikaler Richtung. Durch diese höhere Stoßwahrscheinlichkeit in horizontaler Richtung wird 
dann das Material in dieser Richtung stärker gebündelt. Die laterale Ausdehnung des Brennflecks beträgt in horizontaler und vertikaler Richtung einige $\mathrm{mm}$ bis $\mathrm{cm}$. In der Tiefe wird das Target allerdings nur im Bereich von $\mu \mathrm{m}$ beeinflusst. Damit ist die Stoßwahrscheinlichkeit in Richtung der Targetnormalen am geringsten, so dass das Material vom Target weg beschleunigt wird. Entsprechend wurde dieser Vorgang auch schon für Metalle beschrieben [33]. Das vor dem Metalltarget entstehende Plasma wird ebenfalls vom Target weg beschleunigt. Gegenüber des Targets befindet sich in einem Abstand von $50 \mathrm{~mm}$ das verwendete Substrat (siehe Kap. 2.1.2), auf dem sich dann das Material niederschlägt. Das Substrat wird während der Schichtherstellung rotiert, um eine homogenere Schichtverteilung zu gewährleisten.

In der Literatur wurde bisher beschrieben, dass sich durch die gepulste Laserdeposition keine glatten Schichten herstellen lassen, wenn das Targetmaterial ein schwacher Absorber ist [45, 44, was bei vielen Polymeren der Fall ist. So hat z. B. Poly(methyl methacrylat) (PMMA) bei $\lambda=248 \mathrm{~nm}$ nur einen Absorptionskoeffizient von $\alpha=2 \cdot 10^{2} \mathrm{~cm}^{-1}$ 93. Bei solchen Materialien werden neben einzelnen Ketten oder deren Bruchstücken, Atomen oder Ionen auch größere Tröpfchen (droplets) auf das Substrat übertragen [101].

In dieser Arbeit soll wiederholt werden, wie glatte PMMA-Schichten durch die gepulste Laserdeposition hergestellt werden können. Die Eigenschaften dieser Schichten und deren thermische Stabilität werden hier analysiert und die Härte gezielt beeinflusst.

\subsubsection{Targets und Substrate}

Als Targetmaterial wurde Poly(methyl methacrylat)-Pulver von der Firma Sigma ALDRICH mit einer mittleren Molmasse von $\bar{M}_{w}=996 \mathrm{~kg} \mathrm{~mol}^{-1}$ und einer Ausgangsdichte von $\rho=1,17 \mathrm{~g} \mathrm{~cm}^{-3}$ verwendet. Die mittlere Kettenlänge entspricht somit 9.960 Monomereinheiten ausgehend von der Masse einer Monomereinheit von $100 \mathrm{~g} \mathrm{~mol}^{-1}$. Das PMMA-Pulver wurde unter einem Druck von $p=6 \cdot 10^{7} \mathrm{~N} \mathrm{~m}^{-2}$ eine Stunde bei $120^{\circ} \mathrm{C}$ zu festen Targets gepresst.

Um den Vernetzungsgrad in den deponierten Schichten zu erhöhen, wurde für die Herstellung der Targets das PMMA-Pulver vor dem Pressen zu verschiedenen Anteilen mit pulvrigem Bisphenol A Dimethacrylat (BisDMA) der Firma SIGMA AldRICH vermischt (s. Kap. 6.2). Das Molekulargewicht des BisDMA beträgt $M_{w}=364,44 \mathrm{~g} \mathrm{~mol}^{-1}$. Da dieser Vernetzer eine Schmelztemperatur von nur $72-74{ }^{\circ} \mathrm{C}$ aufweist, wurde dies beim Pressen der Targets entsprechend berücksichtigt und die Heizung während des Pressens nur einige Minuten dazugeschaltet.

Wenn nicht anders angegeben, wurden die Targets für die Schichtdeposition mit Laserfluenzen von etwa $125 \mathrm{~mJ} \mathrm{~cm}^{-2}$ bestrahlt. Aufgrund der schwachen Absorption des PMMA bei der hier verwendeten Wellenlänge zeigen sich Inkubationseffekte, die dazu führen, dass eine konstante Abtragsrate erst nach einer gewissen Pulszahl gewährleistet werden kann (Kap. 4.1). Deshalb und um Verunreinigungen an 
der Targetoberfläche zu beseitigen, wurden die Targets vor der Herstellung mit 500 Laserpulsen „eingeschossen“.

Als Substrate wurden hauptsächlich 1-2 $\mathrm{cm}^{2}$ große Stücke aus Silizium(111)Wafern der Firma CRYSTEC Kristalltechnologie geschnitten. Für die Analysen der chemischen Zusammensetzung der Polymere mit Hilfe der Infrarotspektroskopie (Kap. 2.3.1) wurden wegen der höheren Reflektivität raue, $1 \mathrm{~cm}^{2}$ große Substrate benötigt, die einfach aus einem vorhandenen Aluminium-Blech geschnitten wurden. Die Röntgen-Nahkanten-Absorptions-Spektroskopie (Kap. 2.3.2) wurde in Transmission durchgeführt. Hierfür wurden Siliziumnitrid-Substrate mit einem auf $100 \mathrm{~nm}$ gedünnten Messfenster von der Firma SILSON verwendet, die durchstrahlt werden können.

\subsubsection{Nachbehandlung der Schichten}

Die laserdeponierten Polymerschichten konnten auf verschiedene Weise nachträglich behandelt werden.

- Ohne die hergestellten Schichten aus dem Vakuum holen zu müssen, konnten sie durch einen Strahlungsofen noch in der PLD-Anlage höheren Temperaturen ausgesetzt werden. Dabei konnten die Schichten entweder bei hohen Temperaturen deponiert oder erst nach der Deposition ausgelagert werden.

Der Ofen mit seinen Heizwicklungen ist ein Eigenbau des Hauses, der durch eine Heizregelung der Firma Eurotherm angesteuert wird und bis zu $300^{\circ} \mathrm{C}$ erreichen kann, was für die Polymerschichten ausreichend ist (s. Kap. 5). Die Regelung wird mit einem PID-Algorithmus realisiert und über Zeitfenster mit einem Strom von maximal $I_{\max }=2 \mathrm{~A}$ geheizt. Die Temperatur wurde dabei ständig mit einem Nickel-Chrom/Nickel-Thermoelement zwischen Heizwicklungen und Substrathalter kontrolliert und vor der Herstellung der Schichten mit einem zweiten Thermoelement direkt am Substrathalter kalibriert.

- Die hergestellten Schichten konnten nach dem Ausbau aus der PLD-Anlage an Luft ultravioletter Strahlung ausgesetzt werden.

Dazu wurden die Schichten unter eine Quecksilber-Xenon-Lampe Lightningcure LC5 der Firma HAMAMATsu mit einer Hauptwellenlänge von $\lambda=365 \mathrm{~nm}$ und einer maximalen Leistung von $3500 \mathrm{~W}$ gelegt. Wenn nicht anders angegeben, befanden sich die bestrahlten Proben in einem Abstand von $3 \mathrm{~cm}$ unter dem Ende des Lichtleiters der Lampe. Die bei diesem gewählten Abstand sichtbar bestrahlte Fläche hatte einen Durchmesser von etwa 2,5 cm. Damit konnten die PMMA-Schichten, die auf Si-Substraten einer Größe von $1 \times 2 \mathrm{~cm}^{2}$ deponiert wurden, vollständig ausgeleuchtet werden. 


\subsection{Analyse der Probenmorphologie}

Für die Charakterisierung und Aussagen zur Qualität der deponierten Schichten ist die Untersuchung der entstandenen Oberfläche von großer Bedeutung. Aus diesem Grund wurden mehrere Verfahren verwendet, um Aussagen über z. B. die Homogenität, die Rauigkeit oder die Haftung zum Substrat machen zu können. Aus Ellipsometriemessungen wurden meist die aus der PLD resultierenden Schichtdicken glatter Proben bestimmt sowie Aussagen über den Brechungsindex getroffen. Schichtdicken konnten über die ganze Probengröße mit Hilfe eines Profilometers vermessen werden.

\subsubsection{Rasterelektronenmikroskopie (SEM)}

Die Bilder der Rasterelektronenmikroskopie (Scanning Electron Microscopy, SEM) wurden mit einem Mikroskop vom Typ S360 der Firma CAMBrIDGE Instruments (Wolframkathode, Sekundärelektronendetektor) aufgenommen. An der W-Kathode liegt normalerweise eine Beschleunigungsspannung von $U=20 \mathrm{kV}$ an.

Um die elektrisch isolierenden Polymerschichten ohne Aufladungserscheinungen im SEM untersuchen zu können, wurde in den meisten Fällen vorher eine etwa 30-50 nm dicke Goldschicht mit einem Sputter Coater E5400 der Firma BioRAD aufgebracht.

Um Proben im Querschnitt untersuchen zu können, wurde ein fokussierter Ionenstrahl (focused ion beam, FIB) verwendet, um ein Tiefenprofil freizulegen (s. Abb. 4.12). Verwendet wurde ein Nova Nanolab 600 der Firma FEI. Das Bild wurde mit dem integrierten SEM mit Sekundärelektronendetektor aufgenommen.

\subsubsection{Rasterkraftmikroskopie (AFM)}

Höheninformationen lassen sich besser aus Aufnahmen mittels Rasterkraftmikroskopie (Atomic Force Microscopy, AFM) gewinnen. Um die weichen Polymerproben nicht zu beschädigen, wurde das AFM im Tapping Mode betrieben, wodurch eine berührungsfreie Abbildung der Oberfläche möglich ist. Bei den aus den Abbildungen entnommenen Rauigkeiten handelt es sich um die mittlere Standardabweichung von der als mittleren Höhe definierten Nullebene (Root Mean Square roughness, RMSRauigkeit).

Verwendet wurde hier ein Nanoscope IV Multimode von der Firma VeECo unter normalen Raumbedingungen. Die Bildauswertung wurde mit der dazugehörigen Software in der Version 5.12r3 vorgenommen.

\subsubsection{Auflichtmikroskopie}

Durch ein Auflichtmikroskop erhält man einen großflächigen Überblick über die Probe und es lässt sich z. B. direkt erkennen, ob man eine vollständige Entnetzung der 
Schichten vom Substrat hat, da das blanke Si-Substrat im hier sehr stark reflektiert (s. Abb. 6.14b). Die Proben wurden mit einem Ortholux der Firma LeItz WetzLAR untersucht und die Bilder mit einer Kamera der Firma LumenerA und der dazugehörigen Software LuCam aufgenommen.

\subsubsection{Röntgenreflektometrie (XRR)}

Zur Bestimmung der Schichtdicke und der Grenzflächenrauigkeiten wurde unter anderem die spekuläre Röntgenreflektometrie (X-Ray Reflectivity, XRR) verwendet. Diese hat vor allem bei Schichten, die bei hohen Fluenzen hergestellt wurden, den Vorteil, dass hier der Tröpfchenanteil nicht mitgemessen wird. Die Messungen beziehen sich also nur auf die glatte Komponente dieser Schichten. Die sich ergebenden Interferenzmaxima (Kiessig-Fringes) entsprechen den Reflektionen an der oberen (Vakuum/Schicht) und unteren (Schicht/Substrat) Grenzfläche. Mit Hilfe der Software IMD [109] können durch Anpassung der Fit-Parameter die Schichtdicke, die Dichte und die Rauigkeiten von Schicht und Substrat bestimmt werden.

Für die Messungen wurde ein Vierkreisdiffraktometer vom Typ X'Pert der Firma Phillips mit Kobalt-Röhre $\left(\lambda_{K_{\alpha}}=1,7903 \AA\right)$ und Eisen-Filter im Strahlengang verwendet. Für die Primäroptik wurde ein $\frac{1}{32}^{\circ}$-Divergenzschlitz, 0,04-RadiantSollerschlitze und ein schaltbarer Ni-Abschwächer eingesetzt. Auf der Sekundärseite befanden sich ein 0,2-Radiant-Kollimator und ein 0,05 mm breiter Detektorschlitz, wodurch eine Auflösung von $0,01^{\circ}$ erreicht wurde.

\subsubsection{Ellipsometrie}

Ellipsometrie ist eine sehr sensitive optische Methode, um Informationen über Proben zu erlangen. Hierbei wird ausgenutzt, dass sich der Polarisierungszustand des eingestrahlten Lichts ändert, wenn der Lichtstrahl vom Substrat reflektiert wird. Damit lassen sich dann Rückschlüsse auf verschiedene Schichteigenschaften, z. B. auf die Schichtdicke oder den Brechungsindex ziehen. Detailliert wird dies von Azzam beschrieben [4].

Verwendet wird hier ein Nulling-Ellipsometer $\mathrm{EP}^{3}$ der Firma NAnOfILM. Bestrahlt werden die Proben mit einem Laser der Wellenlänge $\lambda=636 \mathrm{~nm}$. Bei festem Kompensator im Strahlengang rotieren Polarisator und Analysator so, dass ein minimales Signal am Photodetektor ankommt (Nulling). Diese Messung kann für verschiedene Einfallswinkel wiederholt werden. Die gemessenen Daten werden mit einer Modellanalyse, die die optischen Konstanten und Schichtdicken enthält, ausgewertet. Dabei wird für die PMMA-Schichten ein CAUCHY-Algorithmus durchlaufen. 


\subsubsection{Profilometrie}

Der Schichtdickenverlauf über eine ganze Probe, hier über eine große Distanz von $1 \mathrm{~cm}$, wurde mit einem Profilometer aufgenommen. Verwendet wurde hierfür ein Dektak 150 der Firma VEECO mit einem Auflagegewicht von 5 mg.

\subsection{Materialanalyse}

Bei laserdeponierten Polymerschichten stellt sich immer die Frage, inwiefern das Ausgangsmaterial durch die Herstellungsbedingungen verändert wurde. Zur genauen Charakterisierung der deponierten Schicht wie z. B. der chemischen Bindungsverhältnisse wurden mehrere Verfahren angewandt, um den gesamten Bereich von einer einzelnen Bindung bis zur gesamten Länge einer Polymerkette abzudecken. So geben einem z. B. die Infrarotspektroskopie (FT-IR) oder die Röntgennahkantenabsorptionsspektroskopie (NEXAFS) Auskünfte über einzelne Molekülbindungen, die Größenausschlusschromatographie (SEC) Informationen über die Molmassenverteilung des Materials, wobei die SEC aber in der Größenordnung von kleinen Oligomeren oder gar für das Monomer von PMMA noch nicht sensitiv genug ist. Deshalb wurde zusätzlich eine hochaufgelöste Massenfeinanalyse für die Größenordnung von 1-20 Monomereinheiten des MMA durchgeführt. Außerdem ist an die Anlage der Simultanen Thermoanalyse (STA) ein Massenspektrometer gekoppelt. Dieses misst nur Molmassen unterhalb der Größe eines Methyl methacrylats (MMA), also bis zur Größe des Monomers. Die STA selbst lässt Schlüsse auf die chemische bzw. thermische Stabilität des Materials zu.

\subsubsection{Fouriertransformations-IR-Spektroskopie (FT-IR)}

Zur Untersuchung der chemischen Bindungsverhältnisse, und damit der chemischen Zusammensetzung einer Schicht bedient man sich der Fouriertransformations-Infrarotspektroskopie (FT-IR). Bei einer Messung durchläuft die Infrarotstrahlung die komplette Schichtdicke, wird am Substrat reflektiert und durchläuft die Schicht erneut. Damit ergibt sich aus der Messung die Transmission der deponierten Schicht. Die eingestrahlte Energie führt hier nicht zu einer Anregung der Elektronenniveaus, sondern zu Schwingungen und Rotationen der Moleküle oder Molekülteile. Die Grundschwingungen der Moleküle liegen im mittleren Ultrarot-Bereich von $4.000 \mathrm{~cm}^{-1}(2,5 \mu \mathrm{m})$ bis $400 \mathrm{~cm}^{-1}(25 \mu \mathrm{m})$.

$\mathrm{Zu}$ diesen Grundschwingungen gehören sowohl die Valenzschwingungen (stretching vibration), was eine periodische Änderung der Atomabstände bedeutet, als auch die Deformationsschwingungen, bei denen sich die Bindungswinkel periodisch ändern (Abb.2.2). Sind mehr als zwei Atome an der Schwingung beteiligt, wird zwischen der symmetrischen Valenzschwingung $\nu_{\mathrm{s}}$ und der antisymmetrischen Valenzschwingung $\nu_{\mathrm{a}}$ unterschieden, z. B. $\nu_{\mathrm{s}}\left(\mathrm{CH}_{2}\right)$ und $\nu_{\mathrm{a}}\left(\mathrm{CH}_{2}\right)$. Besteht eine Schwingung hauptsächlich 


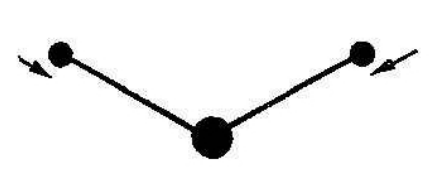

$v_{s}\left(\mathrm{CH}_{2}\right)$

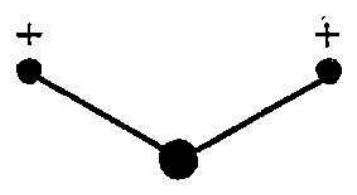

$\gamma_{w}\left(\mathrm{CH}_{2}\right)$

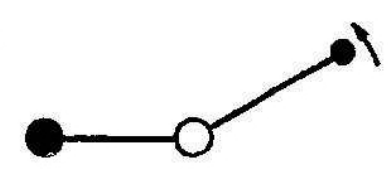

$\delta_{i}(\mathrm{OH})$

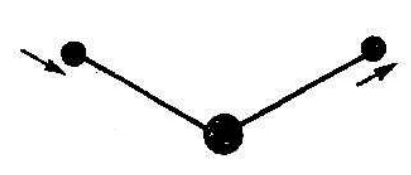

$\mathrm{\nu}_{a}\left(\mathrm{CH}_{2}\right)$

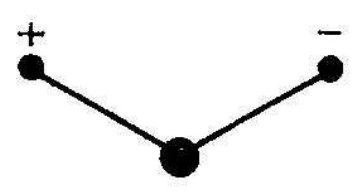

$\gamma_{t}\left(\mathrm{CH}_{2}\right)$

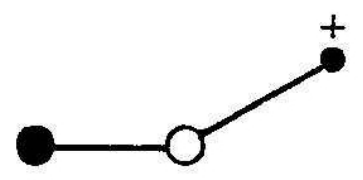

$\gamma_{w}(\mathrm{OH})$

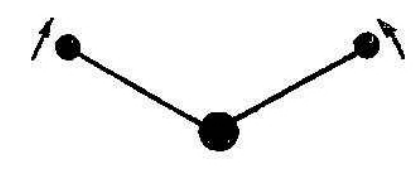

$\delta_{s}\left(\mathrm{CH}_{2}\right)$

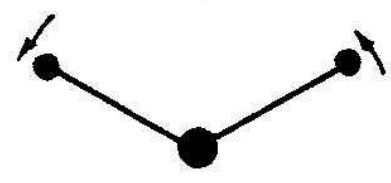

$\gamma_{r}\left(\mathrm{CH}_{2}\right)$

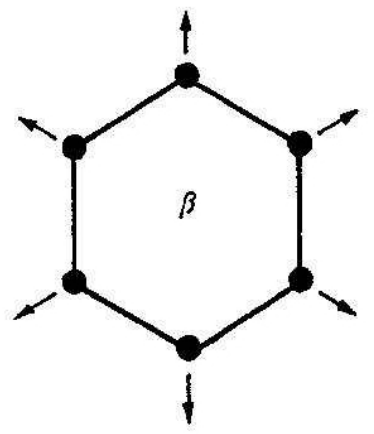

- C-Atom, $\bigcirc-$ O-Atom, - H-Atom

Abbildung 2.2: Bezeichnung einiger Molekülschwingungen nach Dechant [19] 
aus der Änderung von Valenzwinkeln, bezeichnet man dies als Deformationsschwingung $\delta$. Eine spezielle Deformationsschwingung in einer bevorzugten Ebene ist hier z. B. die symmetrische Deformationsschwingung (Scherenschwingung) $\delta_{\mathrm{s}} \operatorname{der} \mathrm{CH}_{2}$ Gruppe, also $\delta_{\mathrm{s}}\left(\mathrm{CH}_{2}\right)$. Die Schwingung in der $\mathrm{COH}$-Ebene würde man mit $\delta_{\mathrm{i}}(\mathrm{OH})$ beschreiben. Gehen Deformationsschwingungen aus einer bevorzugten Ebene heraus, bezeichnet man sie als Waggingschwingungen $\gamma_{\mathrm{w}}$, wobei $\gamma_{\mathrm{w}}(\mathrm{OH})$ die Schwingung senkrecht zur COH-Ebene und $\gamma_{\mathrm{w}}\left(\mathrm{CH}_{2}\right)$ die Schwingung senkrecht zur Ebene der $\mathrm{CH}_{2}$-Gruppe beschreibt. Als Torsions- oder Twistingschwingung $\gamma_{\mathrm{t}}$ werden die Drehschwingungen der $\mathrm{CH}_{2}$-Gruppe um die Winkelhalbierende und als Rockingschwingung $\gamma_{\mathrm{r}}$ die gleichsinnige Schwingung der H-Atome in der Gruppenebene bezeichnet. Wären im Polymer Ringmoleküle vorhanden, könnten diese eine symmetrische Valenzschwingung beschreiben, die man als Ringatmungsschwingung $\beta$ bezeichnet. Beispiele für die einzelnen Schwingungen sind in Abbildung 2.2 dargestellt.

Die Polymerschichten wurden zur Untersuchung auf raue Aluminium-Substrate deponiert. Die Messungen wurden mit einem Gerät vom Typ Tensor 37 mit stickstoffgekühltem MCT-Detektor ( $\mathrm{HgCdTe})$ der Firma BRUKER durchgeführt. Für die Messungen wurde die computergesteuerte Winkelverfahreinheit Auto Seagull der Firma HARRICK zu Hilfe genommen. Es wurde jeweils das Spektrum bei dem Einfallswinkel (zur Oberfläche) für die Auswertung gewählt, bei dem die Ausprägung der Peaks am intensivsten war. Dies war meist bei etwa $75^{\circ}$ der Fall. Die Auflösung der Messungen wurde auf $4 \mathrm{~cm}^{-1}$ festgelegt. Die aufgenommenen Spektren der Polymerschichten wurden durch vorher aufgenommene Hintergrundspektren (leeres Al-Substrat) korrigiert. Um Wasserbanden effektiv zu vermindern, wird der Probenraum des Gerätes während der Messung mit getrockneter Luft gespült.

\subsubsection{Röntgennahkantenabsorptionsspektroskopie (NEXAFS)}

Für weitere strukturelle Untersuchungen einiger Molekülbindungen im Material wurde die Röntgennahkantenabsorptionsspektroskopie (Near-Edge X-ray Absorption Fine Structure, NEXAFS) verwendet. Hierfür wurden auf $100 \mathrm{~nm}$ dicke SiliziumnitridFenster PMMA-Schichten mit einer Dicke von 200-400 nm deponiert, die nur eine Dicke von $100 \mathrm{~nm}$ haben. Somit können diese Substrate durchstrahlt und die Messungen der Schichten in Transmission durchgeführt werden. Die Messungen wurden in Zusammenarbeit mit dem Laser-Laboratorium Göttingen e.V. durchgeführt. Eine genaue Beschreibung der Messapparatur findet sich bei PETH [78].

\subsubsection{Größenausschluss-Chromatographie (SEC)}

Um die Molmassenverteilung von deponierten PMMA-Schichten zu ermitteln, wurde in Zusammenarbeit mit dem Institut für Physikalische Chemie der Universität Göttingen die Größenausschlusschromatographie (Size Exclusion Chromatographie, SEC) angewandt. Hierfür wurden die deponierten Schichten in Tetrahydrofuran 
(THF) gelöst. Diese Polymerlösung wird dann durch ein poröses Trenngel geleitet. Kleinere Moleküle brauchen eine längere Zeit für den Durchlauf durch die Säulen, da sie mehr Volumen zur Verfügung haben als größere Moleküle. Die unterschiedlichen Ankunftszeiten werden mit Standards auf die Molekülgewichte kalibriert. Die Messungen wurden mit einem WATERS-System mit drei PSS-SDV-Säulen mit einer Partikelgröße von $5 \mu \mathrm{m}$ und Porendurchmessern von $10^{4} \mathrm{~nm}, 10^{2} \mathrm{~nm}$ und $10 \mathrm{~nm}$ durchgeführt. Eluent war hierbei ebenfalls THF mit einer Durchflussmenge von $1 \mathrm{ml} \mathrm{min}^{-1}$. Kalibriert wurde dabei mit vier verschiedenen PMMA-Eichstandards (PoLYMER Standard Service, PSS, $\left.800 \mathrm{~g} \mathrm{~mol}^{-1}<M_{P}<1,8 \cdot 10^{6} \mathrm{~g} \mathrm{~mol}^{-1}\right)$.

\subsubsection{Simultane Thermoanalyse (STA)}

Im Messaufbau für die Simultane Thermoanalyse (STA) werden die Messmethoden der Differential-Thermoanalyse (DTA) und der thermogravimetrischen Analyse (TGA) miteinander gekoppelt. Bei der Differential-Thermoanalyse (DTA) wird gemessen, wieviel Wärmemenge einer Probe im Messtiegel zugeführt werden muss, um eine konstante Aufheizrate zu erhalten. Dabei wird die vorher durchgeführte Leermessung des Tiegels vom Signal abgezogen. Die sich daraus ergebenden Peaks zeigen so, je nachdem ob sie ein Minimum oder Maximum in der Messung bilden, einen exothermen bzw. endothermen Vorgang im Probenmaterial. Dies wird verwendet, um die Glasübergangstemperatur des Polymers zu bestimmen.

Zusätzlich wurde der Tiegel mit Probeninhalt während der gesamten Messung gewogen, woraus sich der Masseverlust über die Temperatur ergibt. Mit dieser thermogravimetrischen Analyse (TGA) lassen sich Aussagen über die thermische Stabilität und den Zerfall der Polymerproben machen. An die STA war außerdem ein Massenspektrometer gekoppelt. Hiermit konnten Massen bis maximal $M_{w}=100 \mathrm{~g} \mathrm{~mol}^{-1}$, also nur bis zur Größe des Monomers Methyl methacrylats (MMA) detektiert werden.

Die Messungen wurden in Zusammenarbeit mit dem I. Physikalischen Institut der Universität Göttingen mit einer Heizrate von $1 \mathrm{~K} \mathrm{~min}^{-1}$ an einem Gerät vom Typ STA 449 Jupiter der Firma NeTsCH mit einer Massenspektrometerkopplung durchgeführt.

\subsubsection{Massenspektrometrie}

Um die Lücke zwischen dem eben beschriebenen Massenspektrometer an der STA und der SEC zu schließen, wurden außerdem ESI-MS-Spektren an einem Finnigan LCQ-Ionenfallen Massenspektrometer der Firma Thermo Finnigan in Zusammenarbeit mit dem Institut für Physikalische Chemie der Universität Göttingen aufgenommen. Es können Masse-zu-Ladungs-Verhältnisse im Bereich $200 u \leq m / z \leq$ $2.000 \mathrm{u}$ bei einer Sprühspannung von $4,5 \mathrm{kV}$ und einer Kapillartemperatur von $200^{\circ} \mathrm{C}$ 
detektiert werden. Hierbei können Einfachionisierungen $(z=1)$ und Mehrfachionisierungen $(z \geq 2)$ auftreten, wobei $z$ den Grad der Ionisierung angibt. Die Proben werden in einer Mischung von Dichlormethan und Methanol (3:1) in einer Konzentration von etwa $100 \mu \mathrm{g} / \mathrm{mL}$ gelöst. Etwa 200 Spektren werden zur Verbesserung des Signal-zu-Rauschen-Verhältnisses koaddiert. Detailliert werden solche Messungen von BuBACK beschrieben [9].

\subsection{Mechanische Charakterisierungsmethoden}

Änderungen im Probenmaterial, die nicht oberflächlich sichtbar sind, lassen sich z. B. mit Hilfe von Härtemessungen verdeutlichen.

\subsubsection{Ultramikrohärteprüfung}

Eine grundlegende mechanische Eigenschaft eines Materials ist seine Härte. Hier wurde die Universalhärte von ausreichend dicken Proben und vom Targetmaterial mit einem HV $100 \mathrm{CXYm}$ der Firma Fischer gemessen. Als Indentor wurde eine Diamantpyramide mit quadratischer Grundfläche und einem Öffnungswinkel von $136^{\circ}$ verwendet. Für die Messungen wurde immer eine maximale Kraft von $1 \mathrm{mN}$ vorgegeben, die in einer Zeit von $20 \mathrm{~s}$ angesteuert wird und somit der Verlauf der Eindringtiefe in Abhängigkeit von der Kraft gemessen. Aus den Werten der Maximalkraft mit korrespondierender Eindringtiefe und einem Geometriefaktor für die Indentorspitze ergibt sich dann die Universalhärte [6]:

$$
H U=\frac{1}{26,429} \cdot \frac{F_{\max }}{h^{2}}
$$

Anschließend wurde für $5 \mathrm{~s}$ die Maximalkraft gehalten (Kriechzeit) und danach entsprechend der Eindringkurve in $20 \mathrm{~s}$ die Kraft wieder zurück genommen. 


\section{Ablation und Deposition}

In diesem Kapitel soll zunächst der aktuelle Wissensstand über die gepulste Laserdeposition von Poly(methyl methacrylat)-Schichten zusammengefasst werden. Deshalb wird hier beschrieben, wie es in der vorangegangenen Diplomarbeit erstmals möglich war, sehr glatte PMMA-Schichten mit der gepulsten Laserdeposition herzustellen [70. Die Ablation und entsprechend die Deposition von PMMA wird durch eine Vielzahl von Parametern bestimmt, wovon einige wie z. B. die eingestrahlte Wellenlänge [17] oder die Pulsdauer [57] des verwendeten Lasers unverändert eingesetzt werden (s. Kap. 2.1.1). Dahingegen kann die verwendete Laserfluenz in einem sehr großen Bereich variiert werden, wodurch die Morphologie, die Zusammensetzung und viele weitere Eigenschaften stark beeinflusst werden.

Dass bei sehr hohen Laserfluenzen von einigen $\mathrm{J} \mathrm{cm}^{-2}$ zweikomponentige PMMASchichten entstehen, die einen glatten, vernetzten sowie einen tröpfchenartigen Anteil aufweisen, war schon aus anderen Arbeiten bekannt [104, 87]. Nun wurden erstmals mittels der Laserdeposition bei sehr viel geringeren Fluenzen auch einkomponentige PMMA-Schichten hergestellt, die glatt und tröpfchenfrei sind [69]. Um diese Ablation und Deposition des PMMA (Kap. 3.1) im Detail verstehen zu können, soll hier der ganze realisierbare Laserfluenzbereich mit der Depositionsschwelle als untere Schranke beschrieben werden. Die Depositionsschwelle entspricht der Energiedichte, oberhalb der eine Materialdeposition überhaupt erst möglich ist (Kap. 3.2). Die eingestrahlte Laserfluenz hat dabei auch entscheidenden Einfluss auf die Morphologie der deponierten Schichten (Kap. 3.3), zu der auch die Verteilung der Schichtdicke gehört (Kap. 3.4), und auf die chemischen Eigenschaften (Kap. 3.5).

\subsection{Poly(methyl methacrylat) (PMMA)}

Polymere zeichnen sich durch lange, kettenförmige Moleküle mit entsprechend hoher Molekülmasse und einer immer wiederholenden kleinsten Einheit, dem Monomer, aus. Damit ist die Laserdeposition von Polymeren sehr komplex im Gegensatz zum Abtrag einzelner Atome im Falle von z. B. Metallen. Die Kettenmoleküle sind im Normalfall so groß, dass eine gelegentliche Spaltung bzw. Verbindung von Ketten die Eigenschaften des Polymers kaum verändert. Eine Aufspaltung in die einzelnen Atome ist dagegen absolut unerwünscht, da so keine intakten Polymerschichten auf Substraten deponiert werden können; die Möglichkeit der Rekombination reicht bei einer solchen Zerstörung nicht aus, um das Material wieder zu repolymerisieren. 


\begin{tabular}{|c|c|}
\hline Bindung & Bindungsenergie $[e \mathrm{~V}]$ \\
\hline$\left(\mathrm{CH}_{2}\right)_{2}\left(\mathrm{CH}_{3}\right) \mathbf{C}-\mathrm{COOCH}_{3}$ & $3,1-3,5$ \\
\hline$\left(\mathrm{CH}_{2}\right)\left(\mathrm{CH}_{3}\right)\left(\mathrm{COOCH}_{3}\right) \mathrm{C}-\mathrm{CH}_{2}$ & 3,3 \\
\hline$\left(\mathrm{CH}_{2}\right)_{2}\left(\mathrm{COOCH}_{3}\right) \mathbf{C}-\mathrm{CH}_{3}$ & $3,4-3,5$ \\
\hline$\left(\mathrm{CH}_{2}\right)_{2}\left(\mathrm{CH}_{3}\right) \mathrm{CCO}-\mathrm{OCH}_{3}$ & 4,1 \\
\hline
\end{tabular}

Tabelle 3.1: Ausgewählte Bindungsenergien von C-C- und C-O-Einfachbindungen im PMMA nach MANRING [71]

Damit sind die hohen Temperaturen, die durch den Laserbeschuss an einem stark absorbierenden Metalltarget entstehen, bei Polymeren unbedingt zu vermeiden.

Entscheidend für die gepulste Laserdeposition von Polymerschichten ist somit vor allem die Stärke der Absorption des Targetmaterials. Sie ist abhängig von der eingestrahlten Wellenlänge (für PMMA gezeigt bei SuTCLIFFE [100]), die hier fest bei $\lambda=248 \mathrm{~nm}$ liegt. Während Metalle meist eine sehr hohe Absorption aufweisen [46], kann diese für Polymere je nach Molekülaufbau sehr unterschiedlich sein. Über das Lambert-Beersche Gesetz ist die jeweilige Eindringtiefe $d$ ins Material antiproportional über den Absorptionskoeffizienten $\alpha$ beschrieben:

$$
d=\frac{1}{\alpha} \ln \left(\frac{\Phi(x, y, d)}{\Phi_{0}(x, y)}\right)
$$

Gleichung 3.1 beschreibt die Wechselwirkung von Licht mit Materie als Ein-PhotonProzess. Hierbei ist $\Phi_{0}$ die Schwellenintensität, die mindestens für die Ablation des entsprechenden Materials benötigt wird. Die Eindringtiefe $d$ der Strahlung wächst so logarithmisch mit wachsender Intensität $\Phi$. SRINIVASAN spricht bei der ablativen Photodekomposition von der Beteiligung sowohl eines Ein-Photon-Prozesses, welcher das Abspalten des Monomers MMA und polymere Fragmente mit geringer Molmasse zu verantworten hat, als auch eines Mehr-Photonen-Prozesses, welcher zu Produkten mit hoher kinetischer Energie führt, wie z. B. $C_{2}$ [96]. Nach LIPPERT können für geringe Laserfluenzen Mehr-Photonen-Prozesse vernachlässigt werden [66].

Für $\lambda=248 \mathrm{~nm}$ liegen die Absorptionskoeffzienten von Polymeren für starke UV-absorbierende Polymere, z. B. für Poly(bisphenol A carbonat) (PC) bei $\alpha=$ $1 \cdot 10^{4} \mathrm{~cm}^{-1}$ 61] bzw. $\alpha=1 \cdot 10^{5} \mathrm{~cm}^{-1}$ [60] und für schwache Absorber wie Poly(methyl methacrylat) (PMMA) bei $\alpha=2 \cdot 10^{2} \mathrm{~cm}^{-1}$ [93]. PC zeigt in einem kleinen Bereich der Laserfluenz - direkt oberhalb der Ablationsschwelle - nach der Deposition noch polymeren Charakter [26, 27], während dies für PMMA im gesamten zugänglichen Laserfluenzbereich festgestellt werden konnte. Auf den Einfluss der Laserfluenz soll im Kapitel 3.2 näher eingegangen werden.

Durch solch einen geringen Absorptionskoeffizienten und damit durch eine große Eindringtiefe der Strahlung für PMMA (ca. $150 \mu \mathrm{m}$ [61]) reicht die absorbierte Ener- 

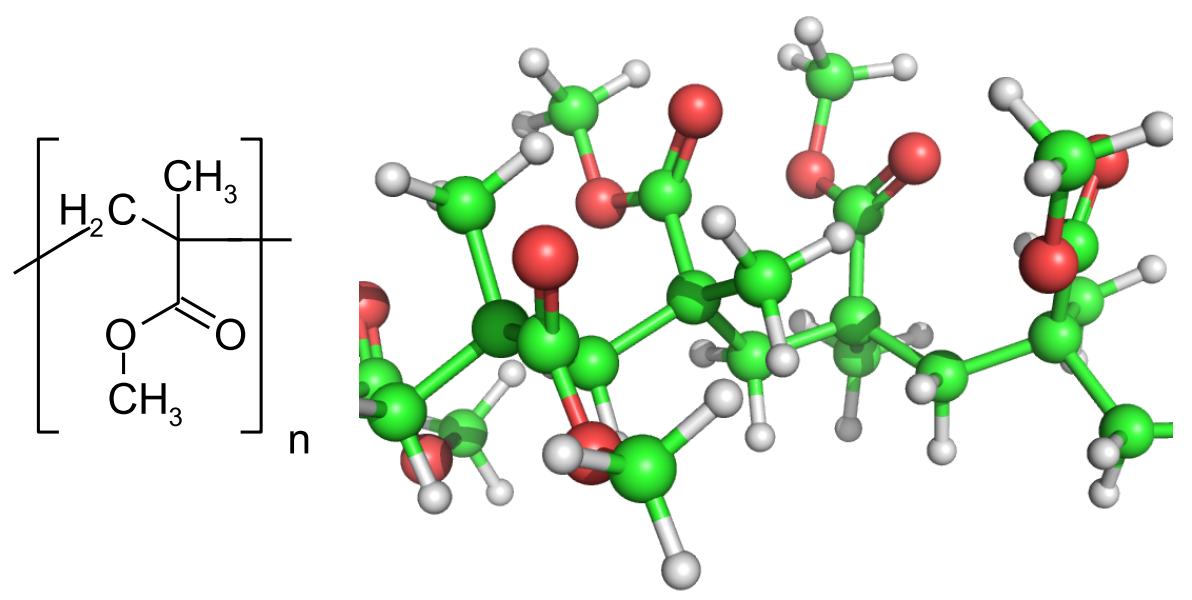

Abbildung 3.1: Strukturformel und Visualisierung von Poly(methyl methacrylat) (PMMA) (C: grün, O: rot, $\mathrm{H}:$ weiß)

gie pro Volumen nicht aus, um einen thermischen Materialabtrag stattfinden zu lassen. Aus der Strukturformel von PMMA (Abb. 3.1) ist ersichtlich, das der hauptsächliche Anteil der Molekülbindungen durch kovalente Einfachbindungen (z. B. C-CBindungen mit einer Bindungsenergie von 3-3,5 eV [71]) gegeben ist. Die Stärke der $\mathrm{C}-\mathrm{C}$-Bindung hängt davon $\mathrm{ab}$, an welcher Position im Molekül sie sich befindet (Tabelle 3.1). Normalerweise ist die einzige Art der Doppelbindungen im PMMA mit entsprechend höherer Bindungsenergie in der Carbonylgruppe lokalisiert $(\mathrm{C}=\mathrm{O}$ mit $\sim 7,3 \mathrm{eV}$ [75]). Zusätzlich finden sich aber im laserdeponierten PMMA verstärkt $\mathrm{C}=\mathrm{C}$-Doppelbindungen, die durch den Depositionsprozess, genauer durch die Inkubation (s. Kap. 4.1), im Material gebildet werden. $\mathrm{C}=\mathrm{C}$-Doppelbindungen haben Bindungsenergien von $6,4 \mathrm{eV}$ [75]. Damit könnten mit der Photonenenergie eines $\mathrm{KrF}$-Excimerlasers von $5 \mathrm{eV}$ die meisten kovalenten Einfachbindungen gebrochen werden. Allerdings sind Chromophore, also die Gruppen in Molekülen, die das Absorptionsverhalten des Polymers charakteristisch beeinflussen, im wesentlichen die Doppelbindungen der Carbonylgruppen [81], weshalb das UV-Licht so gut wie nicht in die Einfachbindungen einkoppelt. Da Doppelbindungen im PMMA lokal relativ selten sind, verteilt sich die Energie über ein großes Volumen, wodurch diese hohen Eindringtiefen im Material zustande kommen.

Die Einstrahlung der Energie in das Material wird dabei hauptsächlich als eine Anregung vom Grundzustand $S_{0}$ in den Singulettzustand $S_{1}$ beschrieben. Dabei sind direkte Anregungen der Bindungen in höhere Zustände für Ein-Photon-Prozesse und Mehr-Photonen-Prozesse zu vernachlässigen [79]. Der angeregte Zustand kann direkt oder über einen Übergang in einen Triplettzustand $T_{1}$ zur Dissoziation führen. Diese beiden Prozesse zählen somit zur photochemischen Ablation 92. Führt die eingestrahlte Energie nicht direkt zu einer Anregung, sondern wird über intramolekulare 
Prozesse auf die nächstliegenden Bindungen oder über intermolekulare Mechanismen in Form von Wärme sogar auf umliegende Ketten weiter verteilt, handelt es sich um photothermische Mechanismen. Dadurch, dass sich die Energie über ein Volumen mehrerer Ketten hinweg verteilt, wird die Temperatur dermaßen erhöht, dass sich das Polymer in diesem Volumenbereich verändern kann. Ist die eingestrahlte Energie trotz der weiten thermischen Ausbreitung lokal hoch genug, können auch hierdurch Bindungen brechen (photofragmentation) [80].

Beide Mechanismen werden in der Literatur kontrovers diskutiert [97, 1, 56]. Aktuelle Übersichten von Dyer [22] oder CHRISEY [15] beschreiben, dass es sich bei einer Bestrahlung mit einer Wellenlänge von $248 \mathrm{~nm}$ um einen zweistufigen Prozess handelt, bei dem beide Mechanismen nacheinander ablaufen.

Die wichtigsten ablaufenden Prozesse bei der Modifikation des PMMA nach LIPPERT sind schematisch in Abbildung 3.2 dargestellt. Hier startet die Zersetzung mit der Anregung der Monomereinheiten. Bei einer Anregung mit Licht von $248 \mathrm{~nm}$ Wellenlänge betrifft dies den Übergang $n \rightarrow \pi^{*}$ der Estergruppe, die Energie koppelt also in die $\pi$-Bindung der Carbonylgruppe ein. Danach folgt die Abspaltung der Seitengruppen bzw. der gesamten Estergruppe, wie in Abbildung 3.2, Schritt 1 zu sehen. Das Aufbrechen der Bindungen neben der Carbonylgruppe ist eine photochemische Reaktion, bekannt als Norrish Typ I-Reaktion oder $\alpha$-Spaltung. In Schritt 2 schließt sich die Abstraktion von Wasserstoff durch die Radikale an und die Ausscheidung von $\mathrm{CO}$ oder $\mathrm{CO}_{2}$ ist zu bemerken. Die aufeinander folgenden Schritte beschreiben die Hauptkettenspaltung, die begleitet wird von der Bildung von Doppelbindungen sowohl an den Kettenenden wie auch im Ketteninnern (Schritt 3), was auch bei der Bestrahlung von PMMA mit einem UV-Laser beobachtet wird. Der letzte Schritt der Zersetzung des PMMA (Abb. 3.2, Schritt 4) ist die endgültige Bildung des Monomers durch Bildung einer Doppelbindung zur $\mathrm{CH}_{2}$-Gruppe. [68, 67, 66]

\subsection{Einfluss der Laserfluenz}

Die Laserfluenz konnte mit dem in dieser Arbeit verwendeten Aufbau von etwa $20 \mathrm{~mJ} \mathrm{~cm}^{-2}$ bis $9 \mathrm{~J} \mathrm{~cm}^{-2}$ variiert werden. Realisiert wurde dieser sehr große Bereich durch eine Kombination mehrerer Methoden. Es konnte die Laserhochspannung durchgefahren (18-26 kV), ein durchlässiger Spiegel als Abschwächer in den Strahlengang gebracht und vor allem die Brennfleckgröße auf der Targetoberfläche verändert werden. Letzteres geschieht durch eine Verschiebung der fokussierenden Linse im Strahlengang, wodurch der Brennfleck auf Größen von $0,02 \mathrm{~cm}^{2}$ bis $2,5 \mathrm{~cm}^{2}$ eingestellt werden kann. Im Folgenden wird gezeigt, dass damit der gesamte interessante Depositionsbereich für PMMA abgedeckt ist.

Wie später in Kapitel 3.3 beschrieben wird, bewirken die geringsten hier eingestrahlten Fluenzen überhaupt keinen Schichtübertrag, in einem kleinen Fluenzbereich findet man glatte Schichten und bei hohen Laserfluenzen, die auch zum Abtrag 


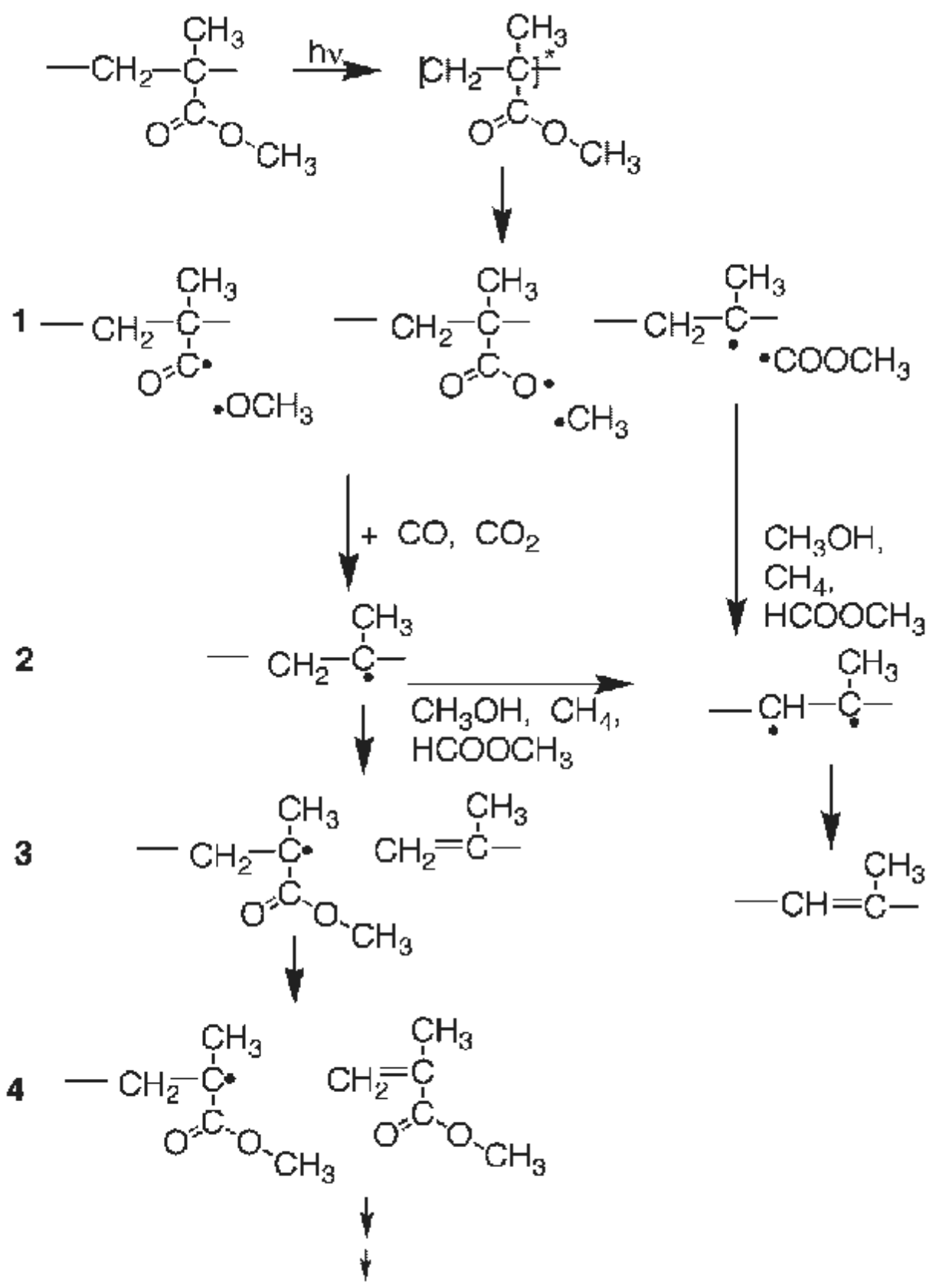

Abbildung 3.2: Modifikation von PMMA durch UV-Einstrahlung: Prozesse zur Photodissoziation nach LIPPERT [68, 67, 66] 


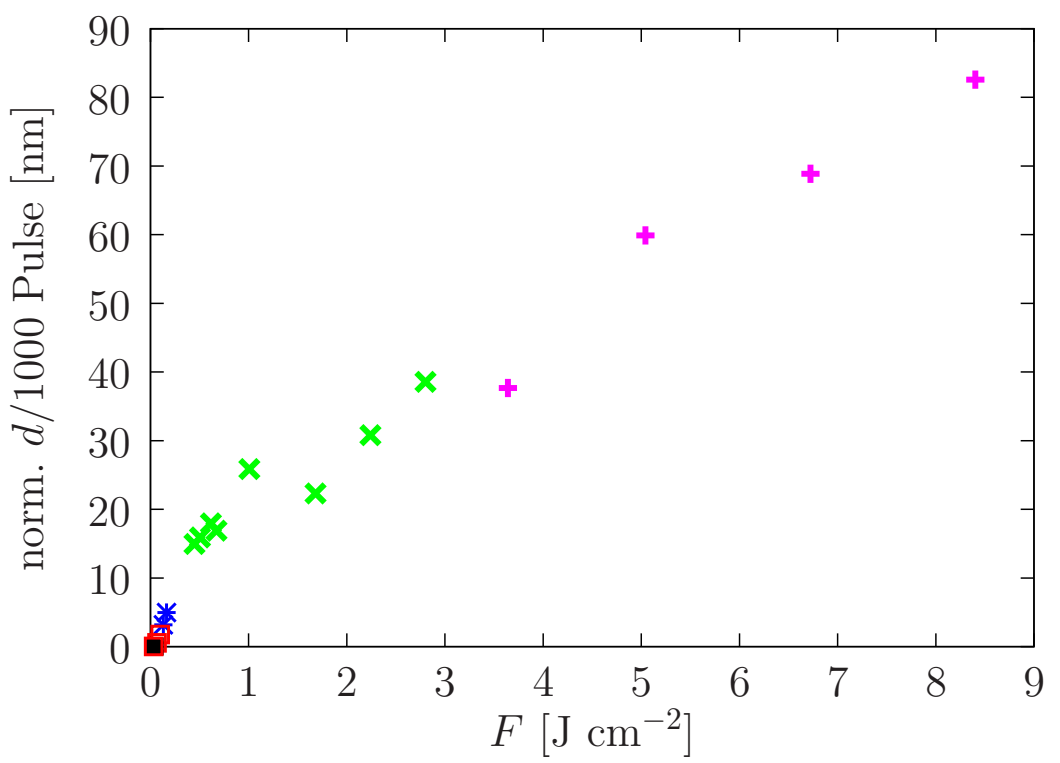

\begin{tabular}{|cl|}
\hline+ & min. Brennfleck \\
× & min. Brennfleck + Abschwächer \\
$*$ & mittl. Brennfleck \\
口 & mittl. Brennfleck + Abschwächer \\
- max. Brennfleck
\end{tabular}

Abbildung 3.3: Depositionsrate in Abhängigkeit von der Laserfluenz F. Die große Spanne von $F$ wird vor allem durch Änderung der Brennfleckgröße erreicht. Hier gilt für den Brennfleck: minimal: $0,7 \mathrm{~mm} \times 2,6 \mathrm{~mm}$, mittel: $4,6 \mathrm{~mm} \times 16,9 \mathrm{~mm}$, maximal: $10,0 \mathrm{~mm} \times 25,0 \mathrm{~mm}$. Die Raten sind hier auf die minimale Brennfleckgröße normiert.

von Metallen verwendet werden, finden sich zusätzlich zu einer stark vernetzten, glatten Komponente auch Tröpfchen (droplets) auf der Polymerschicht. Betrachtet man die Depositionsrate über den gesamten Fluenzbereich normiert auf den kleinstmöglichen Brennfleck von $0,02 \mathrm{~cm}^{2}$ (Abb. 3.3) verringert sich mit geringeren Fluenzen erwartungsgemäß auch die Depositionsrate bis zu deren Verschwinden. Bei hohen Fluenzen ist keine starke Zunahme der Depositionsrate mehr zu bemerken. Diese Sättigung erklärt sich direkt aus dem Ablationsmechanismus am Target. Durch die Bestrahlung des PMMA-Targets bildet sich vor der Targetoberfläche eine gasförmige Schicht der dissoziierten Moleküle, die die weitere Laserpulsstrahlung absorbiert. Dadurch werden die Moleküle vom Target wegbewegt. Mit zunehmender Laserfluenz wird die gasförmige Schicht vor dem Target dichter und schirmt das Target zunehmend ab, bis sich ein Gleichgewicht einstellt.

Die Plasmabildung vor dem Target ist bei Polymeren allerdings viel schwächer ausgeprägt als bei Metallen. Dies liegt daran, dass bei der Bestrahlung von Polymeren ein nennenswerter Abtrag des Materials erst nach dem Ende des Laserpulses stattfindet, wodurch das abgetragene Material mit dem Laserpuls selbst weniger 
wechselwirken kann. Untersuchungen an Luft ergaben in diesem Zusammenhang, dass eine Veränderung des Polymers nach 12 ns einsetzt, ein nennenswerter Abtrag aber erst nach $60 \mathrm{~ns}$ zu verzeichnen ist [95. Da der Abtrag von PMMA durch einen Volumenprozess im Inneren des Materials ohne großartige Wechselwirkungen mit dem umgebenden Medium stattfindet, lassen sich diese Ergebnisse auch auf Prozesse im Vakuum übertragen [51]. Die Geschwindigkeiten des Polymers vom Target weg resultieren hauptsächlich aus der Energie, die durch die Volumenexpansion frei wird.

Der Depositionsprozess für Metalle wird ähnlich beschrieben, wenn auch die Wechselwirkung mit der Laserenergie stärker und damit das Plasma vor dem Target (KNUDSEN-Lage) sehr viel ausgeprägter ist. Metallionen haben somit eine deutlich höhere mittlere kinetische Energie (von etwa $100 \mathrm{eV}$ [34]), weshalb sie eine höhere Geschwindigkeit als die Gasmoleküle eines Polymers haben. Mit einer Zunahme der Raumladungszone im Plasma können die sehr hohen Geschwindigkeiten bei der Expansion nach einem Laserpuls erklärt werden (für Al-Atome bei [36]).

Wie bereits erwähnt, gilt der Kurvenverlauf in Abbildung 3.3 nur für eine Normierung auf eine feste Brennfleckgröße. Da wir aber den Brennfleck vergrößert haben, um geringere Fluenzen auf dem Target zu erreichen, sind dort die Depositionsraten eigentlich viel höher. Der Grund dafür ist, dass mit jedem Puls eine entsprechend größere Oberfläche bestrahlt wird und somit lateral eine größere Menge Material abgetragen werden kann.

Will man den gesamten Fluenzbereich festlegen, der für PMMA interessant ist, sucht man als untere Schranke die kleinste Fluenz, ab der überhaupt erst eine nennenswerte Schichtdeposition stattfindet, die sog. Depositionsschwelle des Materials. Der Bereich um die Depositionsschwelle ist in Abbildung 3.4 dargestellt und hier nicht auf die kleinstmögliche Brennfleckgröße normiert, womit die gezeigten Depositionsraten den tatsächlichen Werten entsprechen. Verwendet wurde bei diesen Messungen einheitlich ein mittelgroßer Brennfleck von $0,8 \mathrm{~cm}^{2}$.

Schon bei sehr geringen Laserfluenzen ist eine Schichtdeposition zu beobachten. Allerdings sind hier die Depositionsraten so gering, dass eine Deposition einer entsprechenden Schichtdicke bei so geringen Fluenzen eine sehr hohe Pulszahl zur Folge hätte. Dadurch wird dieser Fluenzbereich sehr unwirtschaftlich und damit uninteressant. Dass aber überhaupt ein Abtrag bei so geringen Energiedichten möglich ist, liegt hier an einer leichten Erwärmung des Targets. Durch diesen photothermischen Prozess dampft PMMA mit niedrigem Dampfdruck kontinuierlich ab [32], d. h. schon bei geringen Fluenzen erhält man eine geringe, aber konstante Rate bis diese bei Erhöhung der Laserfluenz plötzlich stark ansteigt. Durch eine Extrapolation der gefitteten Geraden an die plötzlich stark ansteigenden Messwerte der Depositionsraten mit zunehmender Laserfluenz findet sich die Depositionsschwelle hier bei $56 \mathrm{~mJ} \mathrm{~cm}^{-2}$. Die Bestimmung der Depositionsschwelle ist nicht einfach, da sich die verwendete Laserfluenz aus der Brennfleckgröße am Target ergibt. Dabei ist aber die Größe des Brennflecks aufgrund der gaußförmigen Energieverteilung der Laserpulse 


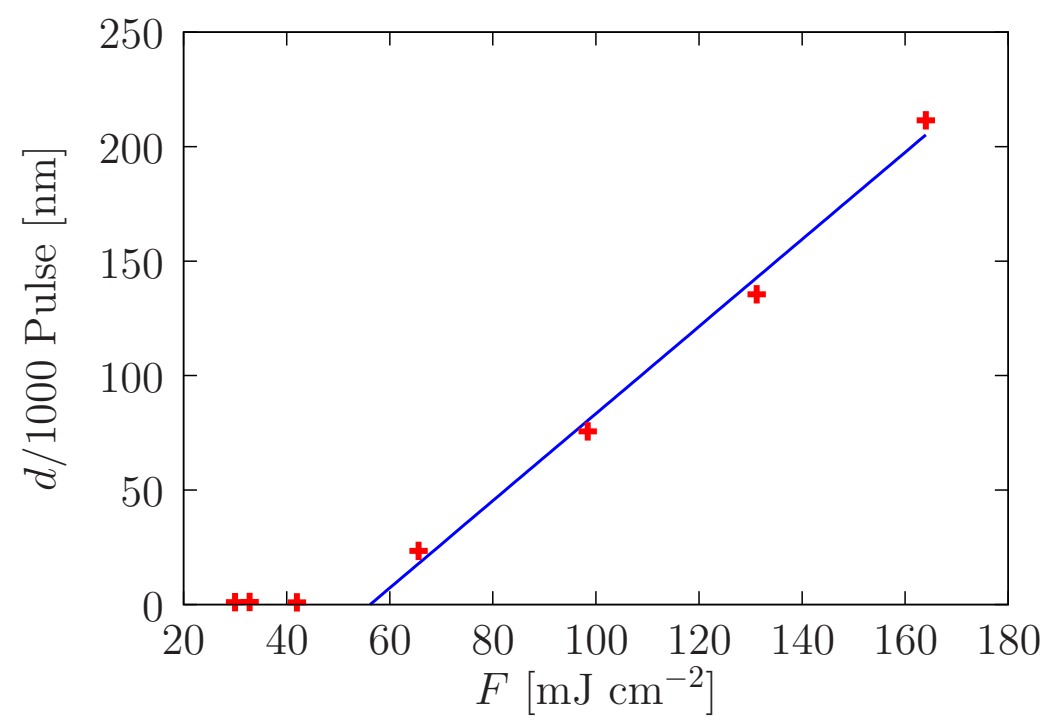

Abbildung 3.4: Depositionsrate in Abhängigkeit von der Laserfluenz $F$ für kleinstmögliche Fluenzen. Zu sehen ist ein schneller Anstieg der Depositionsrate ab der Depositionsschwelle bei $56 \mathrm{~mJ} \mathrm{~cm}^{-2}$. Die Depositionsraten werden durch den größeren Brennfleck mehr als doppelt so hoch wie für sehr hohe Fluenzen, die mit minimalem Brennfleck erreicht werden (vgl. Abb. 3.3).

nur abzuschätzen.

Verwendet wurde hier jeweils der Mittelwert aus den Abmessungen der Verfärbungen auf gebleichtem Papier und auf Thermopapier nach dem Beschuss mit einigen Laserpulsen. Die Werte für die verwendete Laserfluenz haben damit bei kleinsten Werten im Bereich der Depositionsschwelle einen Fehler von etwa $10 \mathrm{~mJ} \mathrm{~cm}^{-2}$, bei sehr großen Werten im Bereich der Laserdeposition von Metallen sogar einen Fehler von etwa $100 \mathrm{~mJ} \mathrm{~cm}^{-2}$. In der Literatur finden sich für scheinbar vergleichbare Herstellungsbedingungen die unterschiedlichsten Depositionsschwellen von PMMA wie z. B. $250 \mathrm{~mJ} \mathrm{~cm}^{-2}$ [61] oder $300 \mathrm{~mJ} \mathrm{~cm}^{-2}$ [103. Dies ist auch ein Anzeichen dafür, wie fehlerbehaftet die Angaben der verwendeten Laserfluenzen sein müssen.

Die hier bestimmten Depositionsraten sind durch den größeren Brennfleck bei Laserfluenzen oberhalb der Depositionsschwelle mehr als doppelt so hoch wie sie für sehr hohe Fluenzen mit minimalem Brennfleck in Abbildung 3.3 erreicht wurden. Da-

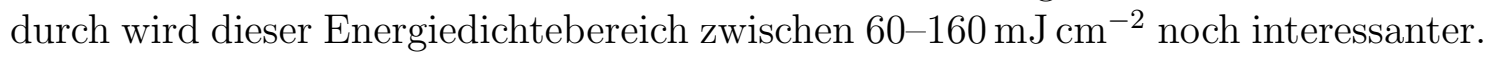

In der Bachelorarbeit von EBERL wurden Schichten aus Poly(ethyl methacrylat) (PEMA) mit den gleichen Herstellungsparametern laserdeponiert [24], wie sie hier für die Deposition von PMMA-Schichten verwendet wurden. Es ergab sich für PEMA eine Depositionsschwelle von etwa $30 \mathrm{~mJ} \mathrm{~cm}^{-2}$. Aufgrund des großen Fehlers bei der Bestimmung der Brennfleckgröße, kann man nicht eindeutig sagen, ob die Depositionsschwelle von PEMA unterhalb der von PMMA liegt. Da die Depositionsschwellen beider Polymere in etwa gleich sind, kann man wohl davon ausgehen, dass 

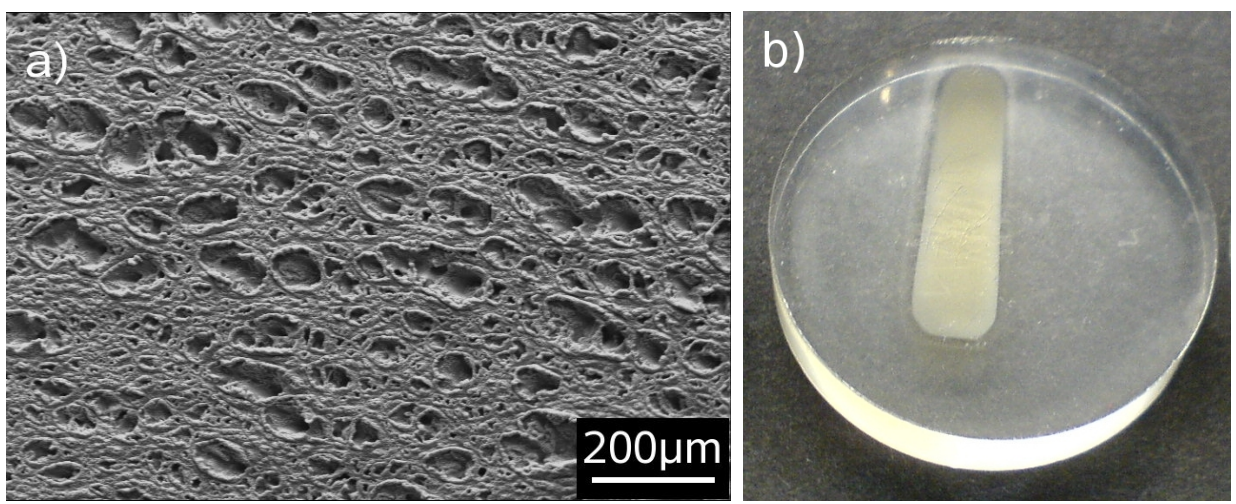

Abbildung 3.5: a) SEM-Aufnahme und b) Fotografie eines PMMA-Targets $(\varnothing=24 \mathrm{~mm})$ nach Laserbeschuss mit Fluenzen unterhalb der Depositionsschwelle (40.000 Pulse bei $20 \mathrm{~mJ} \mathrm{~cm}^{-2}$ ). Hier ist kein Abtrag sondern nur eine Veränderung des Materials zu beobachten. Diese Veränderung zeigt die Inkubation des Polymers, die auch als „Vergilben“ beschrieben wird.

die zusätzliche $\mathrm{CH}_{2}$-Gruppe in der Seitengruppe keine oder kaum Auswirkungen auf die Depositionsschwelle hat.

\subsection{Morphologie}

Die Morphologie der deponierten Schichten lässt sich aus der Betrachtung der bestrahlten Targetoberfläche vorhersagen. In einer SEM-Aufnahme einer mit Fluenzen unterhalb der Depositionschwelle bestrahlten Targetoberfläche ist eine starke Modifikation des Polymers ohne nennenswerten Abtrag zu erkennen (Abb. 3.5 a). Die Targetoberfläche ist hier lediglich aufgeraut und teilweise sind angeschmolzene Vertiefungen zu erkennen. Außerdem wird über die gesamte Ausdehnung des Brennflecks hinweg eine Verfärbung der bestrahlten Oberfläche von farblos transparent nach hellgelb bis gelblich braun festgestellt (Abb. 3.5 b). Die chemische Komposition des Targetmaterials wird somit durch die Bestrahlung mit einer Wellenlänge von $248 \mathrm{~nm}$ modifiziert. Diese sog. Inkubation, die sich vor allem über die Bildung von Doppelbindungen im Material auszeichnet, wird auch als „Vergilben“ des Materials bezeichnet und wird später in Kapitel 4.1 näher untersucht.

Im Laserfluenzbereich direkt oberhalb der Depositionsschwelle (hier etwa 90$160 \mathrm{~mJ} \mathrm{~cm}^{-2}$ ) wird eine gute Ablation vom Target festgestellt (Abb. 3.6). Dies ist daran zu erkennen, dass der bestrahlte Bereich vollständig aufgeschmolzen ist und ein starker Abtrag stattgefunden hat (Abb. 3.6 b). Dadurch hat sich eine Vertiefung auf der Targetoberfläche gebildet, die in der Mitte des Brennflecks ein sehr glattes Profil zeigt (Abb. 3.6a). Trotz des massiven Materialabtrags entstehen an der Targetoberfläche kaum Rauigkeiten oder gar zapfenförmige Strukturen, wie sie für 

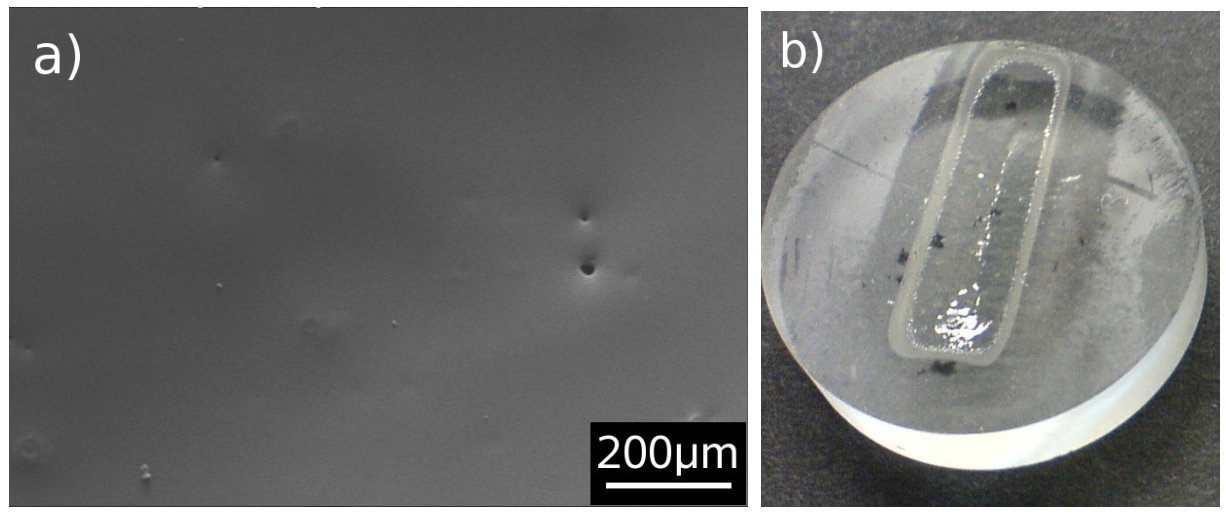

Abbildung 3.6: a) SEM-Aufnahme und b) Fotografie eines PMMA-Targets ( $\varnothing=24 \mathrm{~mm}$ ) nach mehreren Tausend Laserschuss mit Fluenzen bis zu $160 \mathrm{~mJ} \mathrm{~cm}^{-2}$.

die Laserablation verschiedenster Materialien ansonsten typisch sind [14]. Die gelbe Färbung des Targets, die durch die Inkubation hervorgerufen wurde (s. Abb. 3.5b), ist hier nur noch im Randbereich zu erkennen.

Dieser Fluenzbereich, in dem das bestrahlte Target vollständig glatt ist, ist zu höheren Laserfluenzen hin stark begrenzt. Ab Fluenzen von etwa $160 \mathrm{~mJ} \mathrm{~cm}^{-2}$ verändert sich die Targetoberfläche, indem einzelne Löcher auftreten, wie sie schon in Abbildung 3.6 a ansatzweise zu erkennen sind (s. Abb. 3.8).

Entsprechend der Ablation am Target mit glatter Oberfläche lassen sich auf einem gegenüber positionierten Si-Substrat glatte, tröpfchenfreie Schichten deponieren (Abb. 3.7 a). Auch bei großen Schichtdicken von mehreren $100 \mathrm{~nm}$ haben die Schichten Rauigkeiten von unter $1 \mathrm{~nm}$. Während sich durch Röntgenreflektometriemessungen mit angepassten IMD-Fits die RMS-Rauigkeit $\mathrm{zu} \sigma_{\mathrm{RMS}}=0,7 \mathrm{~nm}$ ergibt [69], liegt die RMS-Rauigkeit aus AFM-Messungen sogar nur bei $\sigma_{\text {RMS }}=0,3 \mathrm{~nm}$ (Abb. 3.7b). Die Rauigkeiten, die aus XRR-Messungen bestimmt wurden, sind damit also etwa doppelt so hoch wie die sich aus AFM-Messungen ergebenden Rauigkeiten. Der Grund hierfür ist, dass mit dem AFM nur die lokale Rauigkeit eines vergleichsweise kleinen Bildausschnitts (im $\mu \mathrm{m}^{2}$-Bereich) bestimmt wird, während bei der Reflektometrie über eine makroskopische Fläche (bis $\mathrm{zu} 2 \mathrm{~cm}^{2}$ ) gemittelt wird und so auch sehr langwellige Rauigkeiten, die sich durch Inhomogenitäten oder leichte Krümmungen des Substrats ergeben, gemessen werden 65].

Bei sehr hohen Laserfluenzen von mehreren $\mathrm{J} \mathrm{cm}^{-2}$, wie sie auch für die Ablation von Metallen ausreichen, ist der gesamte beschossene Bereich aufgeschmolzen und vollständig mit Löchern und blasen- oder tröpfchenartigen Erhöhungen übersäht (Abb. 3.8 a). Im Querschnitt, für den das Target einfach bei Raumtemperatur durchgebrochen wurde, ist zu erkennen, dass das Targetmaterial nach dem Abtrag der oberen Schichten noch bis zu $10 \mu \mathrm{m}$ tief unterhalb der Oberfläche verändert wurde (Abb. 3.8b). Diese langreichweitige Veränderung des Materials beschreibt 

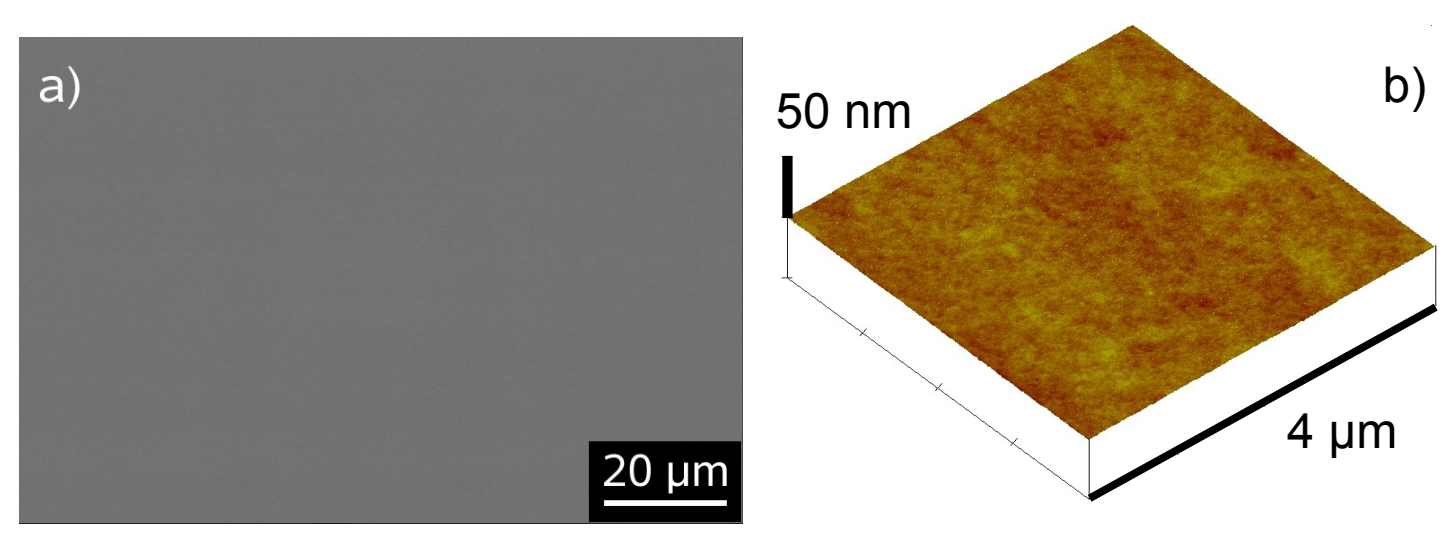

Abbildung 3.7: a) SEM-Aufnahme einer PMMA-Schicht hergestellt mit 8.000 Pulsen bei $98 \mathrm{~mJ} \mathrm{~cm}^{-2}$ und b) AFM-Aufnahme einer PMMA-Schicht hergestellt mit 5.000 Pulsen bei $113 \mathrm{~mJ} \mathrm{~cm}^{-2}$. Die sich ergebende homogene Schicht hat eine RMS-Rauigkeit von $\sigma_{\mathrm{RMS}}=$ $0,3 \mathrm{~nm}$, was einer Rauigkeit eines polierten Si-Wafers entspricht.

eindeutig PMMA als schwachen Absorber für eine Wellenlänge von $248 \mathrm{~nm}$. Die Löcher auf der Oberfläche des beschossenen PMMA-Targets zeigen sich im Querschnitt als Hohlräume unter der Oberfläche, die teilweise nach oben geöffnet sind. Nicht geöffnete Hohlräume bilden auf der Oberfläche Blasen. Dies zeigt, dass sich unter der Oberfläche das vom Laserpuls aufgeheizte Material stellenweise ausgedehnt hat, wodurch Druck unter der Oberfläche ausgeübt wurde. Der Grund dafür ist eine durch Laserbeschuss hervorgerufene Reduktion einzelner Makromoleküle zu kleineren Molekülen, die nun ein größeres Volumen beanspruchen und somit hohe Drücke im Target erzeugen [104]. So benötigt z. B. MMA $30 \%$ mehr Platz als die gleiche Einheit im Makromolekül [35]. Durch Explosion dieser Hochdruckblasen wird der Oberflächenanteil abgesprengt und der Inhalt aus den Blasen befördert, was kleine Degradationsprodukte mit teilweise radikalischem Charakter und Ablationsprodukte mit reduzierter Molmasse freisetzt [104].

SingH et al. beschreiben die Entstehung der Löcher mit dem Subsurface Heating Effect [90]. Hierbei ist die Temperatur an der Targetoberfläche durch die Verdampfung von Material etwas niedriger als in dem Bereich unterhalb der bestrahlten Oberfläche. Dies gilt insbesondere bei schwach absorbierenden Polymeren, zu denen auch das PMMA gehört, bei dem die Laserenergie insgesamt erst sehr tief unterhalb der Oberfläche absorbiert wird. Dort siedet das Material und die etwas kältere Oberfläche kann zerreißen, was durch die Öffnungen in der Targetoberfläche des PMMA bemerkbar wird. Aus diesen Öffnungen wird das heiße, flüssige Material aus dem Target gelöst, zieht sich aufgrund der Oberflächenspannung zu kleinen Tröpfchen zusammen und fliegt weg vom Target auf das gegenüberliegende Substrat.

Dies spiegelt sich auf den dazugehörigen deponierten Schichten in Form von zwei 

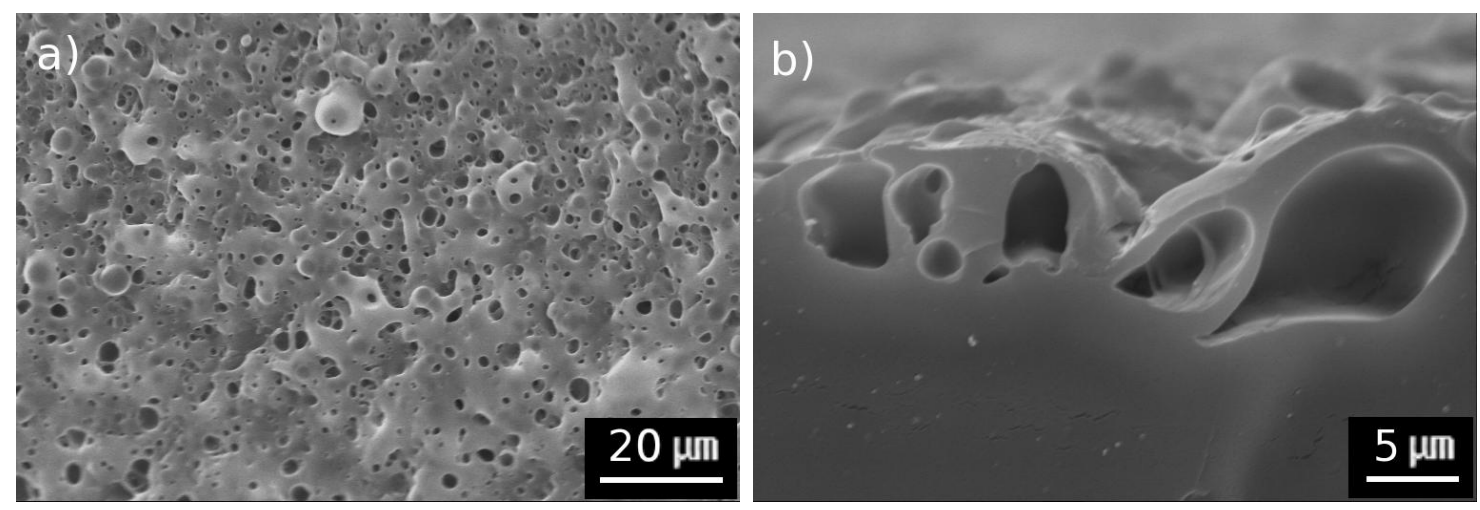

Abbildung 3.8: Target a) in Draufsicht und b) im Querschnitt nach Beschuss mit 800 Pulsen pro Position bei $5 \mathrm{~J} \mathrm{~cm}^{-2}$.

Komponenten - Tröpfchen und einer glatten Schicht - wieder (Abb. 3.9a). Die Tröpfchen sind bei Aufsicht als meist kreisförmige Strukturen zu erkennen. Sehr kleine Tröpfchen haben im Ganzen eine eher halbkugelförmige Erscheinung, während größere Tröpfchen sehr flach im Verhältnis zu ihrem Durchmesser auf dem Substrat zu finden sind. Die unterschiedlichen Tröpfchenformen entstehen aufgrund ihrer unterschiedlichen Temperaturen bzw. Abkühlgeschwindigkeiten bei der Deposition. Ist die Abkühlrate hoch oder die Temperatur eines Tröpfchens beim Auftreffen auf dem Substrat gering, entstehen halbkugelförmige Tröpfchen. Bei größeren Tröpfchen handelt es sich aufgrund ihrer Masse um einen langsameren Abkühlungsprozess, wodurch die beim Auftreffen noch flüssigen Polymertröpfchen durch ihre Aufprallgeschwindigkeit erst flachgedrückt werden, bevor sie erstarren.

Durch XRR-Messungen wird bei dieser Art von Schichten nur der glatte Schichtanteil gemessen, da diese Messmethode für die Tröpfchen nicht sensitiv ist. Die in Kapitel 3.2 beschriebenen Schichtdicken beziehen sich somit nur auf den glatten Anteil der bei hohen Fluenzen deponierten Schichten. In AFM-Messungen sind die Tröpfchen dagegen der dominante Anteil. Durch sie wird die Schicht insgesamt sehr ungleichmäßig, was eine sehr hohe Rauigkeit zur Folge hat. Da die Schichten in diesem Fluenzbereich sehr inhomogen sind, hängt die Rauigkeit stark vom gewählten Bildausschnitt und von der gewählten Bildgröße ab. Typischerweise ergeben sich hier RMS-Rauigkeiten von mehreren Nanometern (Abb. 3.9 b), was für dieses Beispiel eine RMS-Rauigkeit von $\sigma_{\mathrm{RMS}}=6,3 \mathrm{~nm}$ und damit einen mehr als $20 \mathrm{mal}$ höheren Rauigkeitswert ergibt als bei glatten Schichten, die kurz oberhalb der Depositionsschwelle hergestellt wurden. 

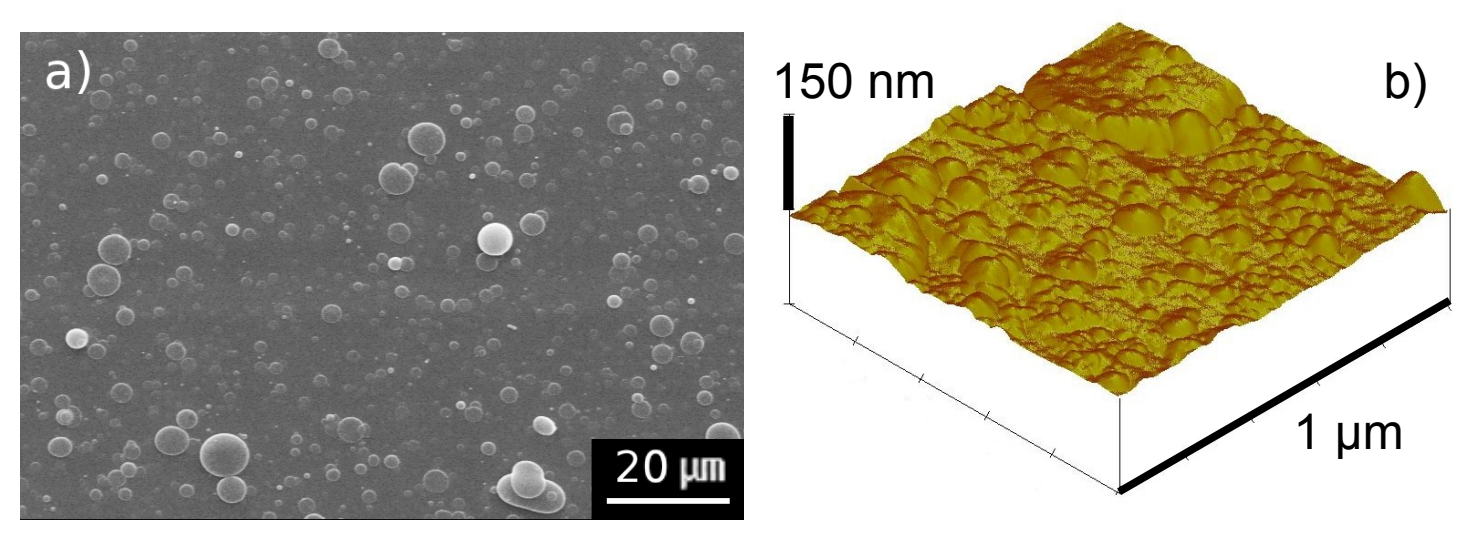

Abbildung 3.9: a) SEM-Aufnahme einer PMMA-Schicht hergestellt mit 500 Pulsen bei $5 \mathrm{~J} \mathrm{~cm}^{-2}$ und b) AFM-Aufnahme einer PMMA-Schicht hergestellt mit 1.000 Pulsen bei $3,6 \mathrm{~J} \mathrm{~cm}^{-2}$. Durch die entstandenen Tröpfchen ergibt sich eine inhomogene Schicht, die für diesen Bildausschnitt eine hohe RMS-Rauigkeit von $\sigma_{\mathrm{RMS}}=6,3 \mathrm{~nm}$ aufzeigt.

\subsection{Schichtdickenverteilung}

Hohe Temperaturen und hohe Stoßwahrscheinlichkeiten im Plasma, welches sich durch die Bestrahlung vor dem Polymertarget bildet, führen zu einer stark gerichteten Materialverteilung, wie sie auch für PLD von Metallen üblich ist [54]. Durch die Stoßprozesse einzelner Atome und Ionen wird dabei der Hauptteil des abgetragenen Materials unabhängig vom Einstrahlwinkel des Lasers senkrecht vom Target ablatiert. Durch eine entsprechende Positionierung des Substrats (s. Kap. 2.1.1) erhält man ein Maximum der Schichtdicke in der Substratmitte. Die deponierte Schichtdicke fällt somit von der Substratmitte zu den Seiten hin gleichmäßig ab und lässt sich mit einer $\cos ^{n} \varphi$-Funktion anfitten. Der Exponent $n$ ist dabei ein Maß für die Bündelung des Plasmas, wobei die Bündelung mit anwachsendem $n$ stärker wird. Bei einem reinen Verdampfungsprozess unter idealen Bedingungen würde man eine $\cos ^{4} \varphi$-Funktion erhalten, da in diesem Fall die momentanen Abtragsraten sehr gering sind und so Stöße im abgetragenen Material kaum vorkommen; dadurch kann sich das Material ungebremst in alle Raumrichtungen ausbreiten.

Bei der Laserablation ergeben sich normalerweise durch die viel höhere, eingebrachte Energie viele Stöße im Plasma vor dem Target. Die Stoßwahrscheinlichkeit hängt dabei stark mit der Ausdehnung des Brennflecks und der Abtragstiefe im Material zusammen. Das Plasma senkrecht zur Targetoberfläche ist sehr viel dünner als dessen laterale Ausdehnung, wodurch auch die Stoßwahrscheinlichkeit in senkrechter Richtung sehr viel geringer ist als parallel zur Targetoberfläche. Durch Stöße in einer Raumrichtung wird die Geschwindigkeit der Teilchen in diese Richtung herabgesetzt. Aus diesem Grund ist also die Geschwindigkeitsverteilung senkrecht zur 

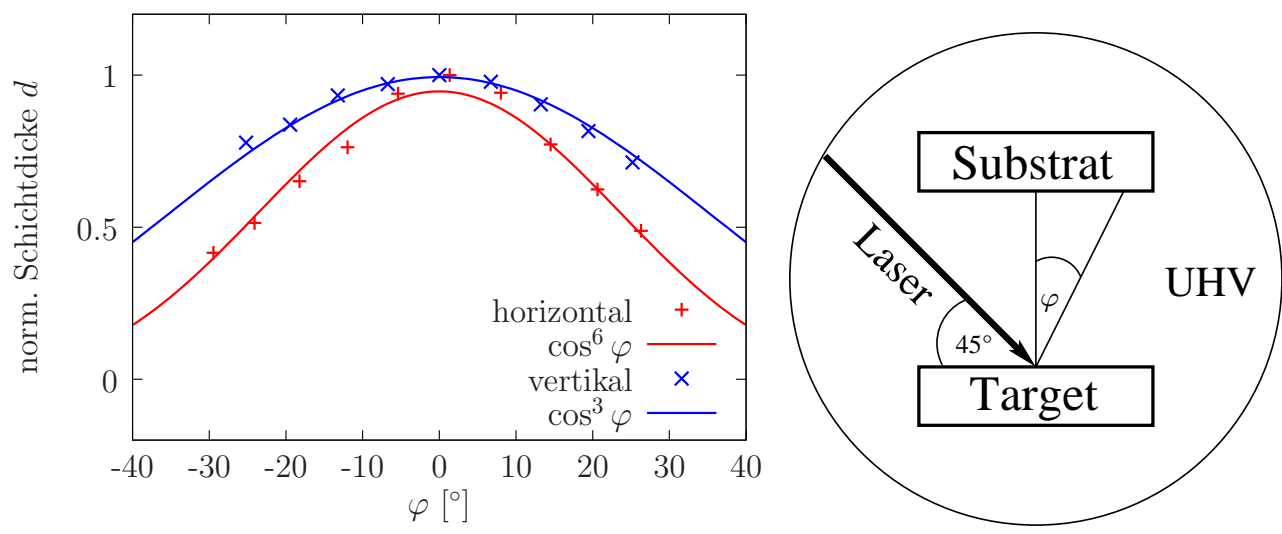

Abbildung 3.10: Schichtdickenverteilung aus IMD-Fits an XRR-Messungen (links): Die Probe wurde mit 500 Pulsen bei $5 \mathrm{~J} \mathrm{~cm}^{-2}$ deponiert und das Substrat wurde bei der Herstellung nicht rotiert, um die Auswirkungen der ungleichen Brennfleckausdehnung zu zeigen. Skizze zur Erklärung des Winkels $\varphi$ zur Targetnormalen (rechts): Schnittpunkt des Substrats mit der Targetnormalen entspricht $\varphi=0^{\circ}$, Lasereintritt in die UHV-Kammer bei $\varphi=-45^{\circ}$. 70

Targetoberfläche am größten und das ablatierte Material wird so vom Target in Richtung des gegenüberliegenden Substrats wegbewegt. Ein Großteil des Materials wird dann auf das Substrat deponiert, da durch die hohe Stoßwahrscheinlichkeit des Plasmas in lateraler Ausdehnung das Material stark gebündelt wird. Bei Metallen ergeben sich für den $\cos ^{n} \varphi$-Fit Werte von bis zu $n=40$ [34]. Tendenziell erwartet man bei Polymeren, besonders bei schwächeren Absorbern wie z. B. dem PMMA, aufgrund der höheren Eindringtiefe der Energie in das Material und dem damit verbundenen geringeren Abtrag, dass die Stoßwahrscheinlichkeiten im Plasma geringer sind und damit die Verteilung des Materials auf dem Substrat breiter wird, wodurch sich ein kleinerer Exponent $n$ ergeben sollte.

Betrachtet man die Schichtdickenverteilung der Tröpfchenschichten, die bei hohen Fluenzen mit entsprechend kleinem Brennfleck von $0,02 \mathrm{~cm}^{2}$ hergestellt wurden, so bedient man sich XRR-Messungen, die mit Hilfe des Programms IMD angefittet wurden (s. Kap. 2.2.4), um nur die Schichtdicke der glatten Komponente ohne Tröpfchen zu ermitteln. Um die Auswirkungen des rechteckigen Brennflecks einmal zu verdeutlichen, wurde hier das Substrat während der Deposition nicht rotiert. Abhängig von der horizontalen bzw. vertikalen Messrichtung ergeben sich Exponenten von $n_{\text {hor }}=6$ bzw. $n_{\text {ver }}=3$ [70] (Abb. 3.10links). Die horizontalen Messwerte beschreiben die Schichtdickenverteilung in der Einfallsebene des Lasers und die vertikalen Messwerte die Verteilung senkrecht dazu. Die beiden Richtungen unterscheiden sich aufgrund der unterschiedlichen Brennfleckausdehnung in diesen Richtungen; der Brennfleck ist in der Horizontalen etwa um ein Vierfaches breiter. Die Abweichung zur Targetnormalen wird durch den Winkel $\varphi$ beschrieben (Abb. 3.10 rechts), 


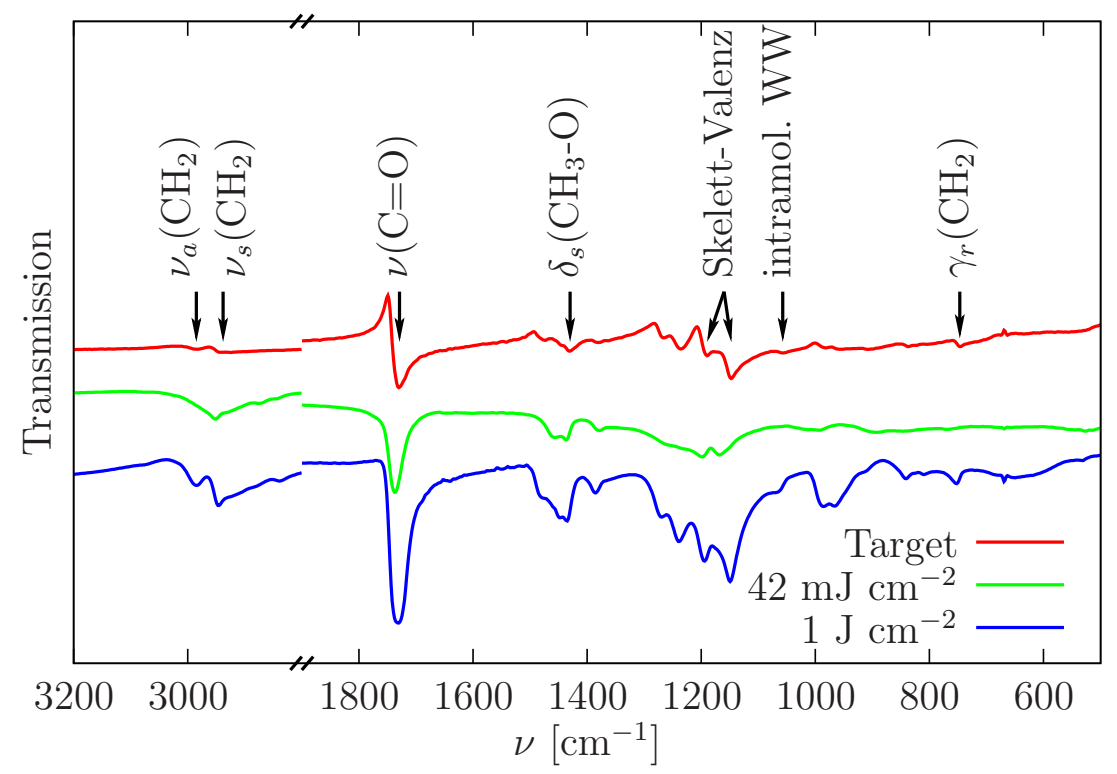

Abbildung 3.11: IR-Spektren eines PMMA-Targets und zweier PMMA-Schichten hergestellt mit 36.000 Pulsen bei $42 \mathrm{~mJ} \mathrm{~cm}^{-2}$ bzw. 6.000 Pulsen bei $1 \mathrm{~J} \mathrm{~cm}^{-2}$. Charakteristische Schwingungsbanden des PMMA sind eingezeichnet [19].

wodurch die Darstellung unabhängig vom Target-Substrat-Abstand während der Deposition wird. Durch die sehr kleine Brennfleckgröße, wie sie auch für Metalle verwendet wird, erhält man hier die vorhergesagte breite Schichtdickenverteilung, wie sie auch für aufgedampfte Materialien vorkommt. Dies lässt sich erklären durch die im Vergleich zu Metallen recht geringe Geschwindigkeit des Plasmas weg vom Target, die hauptsächlich aus der Energie durch Volumenexpansion hervorgeht.

\subsection{Chemische Eigenschaften}

Die chemische Struktur der laserdeponierten PMMA-Schichten wurde mit Fouriertransformations-Infrarotspektroskopie (FT-IR) untersucht (Abb. 3.11). Die Schichten weisen dabei unabhängig von der verwendeten Laserfluenz in den IR-Spektren Vibrationsbanden auf, die identisch sind mit den Banden des Ausgangsmaterials [19]. Die wichtigsten Banden sind in Tabelle 3.2 aufgelistet. Besonders ist in den Messungen die $\mathrm{C}=\mathrm{O}$-Streckschwingung bei $\nu=1729 \mathrm{~cm}^{-1} \mathrm{zu}$ erkennen. Man erhält also unabhängig von der verwendeten Laserfluenz bei der Deposition PMMASchichten mit polymerem Charakter und ähnlich intakter chemischer Zusammensetzung, wobei die Übereinstimmung der IR-Spektren bemerkenswert gut ist. Die Skelett-Valenzschwingungen bei $1190 \mathrm{~cm}^{-1}$ und $1147 \mathrm{~cm}^{-1}$ sind besonders in der bei höheren Fluenzen hergestellten Tröpfchenschicht ausgeprägt. Diese Banden werden 


\begin{tabular}{cl}
\hline Wellenzahl $\nu\left[\mathrm{cm}^{-1}\right]$ & Zuordnung und Art der Schwingung \\
\hline 2985 & $\nu_{a}\left(\mathrm{CH}_{2}\right):$ antisymmetrische Valenzschwingung \\
2938 & $\nu_{s}\left(\mathrm{CH}_{2}\right):$ symmetrische Valenzschwingung \\
1729 & $\nu(\mathrm{C}=\mathrm{O})$ : Streckschwingung \\
1430 & $\delta_{s}\left(\mathrm{CH}_{3}-\mathrm{O}\right):$ symmetrische Deformationsschwingung \\
& um den Valenzwinkel \\
1190,1147 & Skelett-Valenzschwingungen gekoppelt mit inneren \\
& CH-Deformationsschwingungen \\
1057 & intramolekulare Wechselwirkung \\
747 & $\gamma_{r}\left(\mathrm{CH}_{2}\right)$ gekoppelt mit Skelett-Valenzschwingung \\
\hline
\end{tabular}

Tabelle 3.2: PMMA-Vibrationsbanden in den IR-Spektren (bestimmt nach DECHANT [19])

in den glatten Schichten, die im Bereich der Depositionsschwelle hergestellt wurden, schwächer, da hier die Kettenlänge des PMMA etwas stärker verkürzt ist. Das Ausgangsmaterial zeigt hier schwache Banden, da aufgrund der Dicke der Targets keine genauen Hintergrundmessungen durchgeführt werden konnten.

Nachdem hier nun der aktuelle Wissensstand über die gepulste Laserdeposition von glatten PMMA-Schichten zusammengefasst wurde, soll nun im Rahmen dieser Arbeit unter anderem die Charakterisierung dieser Schichten fortgeführt und ihre Stabilität und Anwendbarkeit diskutiert werden. 


\section{Charakterisierung glatter PMMA-Schichten}

In diesem Kapitel soll das Wachstum glatter Poly(methyl methacrylat)-Schichten weiter diskutiert bzw. mehr auf die Struktur und die Eigenschaften der deponierten Schichten eingegangen werden. Um also die Ablation und Deposition des Polymers im Detail weiter verstehen zu können, müssen Prozesse wie die Inkubation, die die Strukturänderung des Polymers ohne Ablation des Materials beschreibt (Kap. 4.1), näher untersucht werden. Die Morphologie der danach deponierten glatten PMMASchichten wird weiter diskutiert, indem die Schichtdickenabhängigkeit (Kap. 4.2) und die Schichtdickenverteilung (Kap. 4.3) näher untersucht werden. Außerdem lassen sich durch eine genaue Betrachtung der chemischen Modifikationen Rückschlüsse auf die Vorgänge während der Laserdeposition ziehen (Kap. 4.4). Anschließend werden die mechanischen Eigenschaften anhand der Universalhärten der glatten Schichten, der Tröpfchenschichten und der Targets diskutiert und miteinander verglichen (Kap. 4.5).

\subsection{Inkubation}

Das Modell von ARnold beschreibt die Ablation von Polymeren als eine Kombination eines photochemischen Volumenmodels und eines thermischen Oberflächenmodels [3]. Bei der ersten Modifikation des PMMA durch UV-Einstrahlung scheint ein photochemischer Mechanismus vorherrschend zu sein [58. Hierbei verändert sich zwar das Polymer stark durch die auftreffenden Photonen, eine Ablation des Materials findet aber noch nicht statt. Diese Materialveränderung fällt besonders bei niedrigem Photonenfluss (z. B. niedrige Fluenzen unterhalb der Depositionsschwelle des Polymers oder bei Sonnenlicht) auf, da sie hier klarer von den Folgeprozessen zu trennen ist. Diese Inkubation, die sich vor allem über die Bildung von Doppelbindungen im Material auszeichnet, wurde bereits als „Vergilben“ des Materials identifiziert, was durch die Aufnahme eines bestrahlten PMMA-Targets mit sehr geringen Laserfluenzen ersichtlich wurde (s. Abb. 3.5).

Die $\mathrm{C}=\mathrm{C}-$ Doppelbindungen, die durch den Inkubationsprozess im PMMA entstanden sind, sollten in Infrarotspektren bei $1643 \mathrm{~cm}^{-1} \mathrm{zu}$ finden sein. Diese Schwingungsbande konnte aber bei den hier durchgeführten FT-IR-Messungen nicht gefunden werden (s. Abb. 3.11). 


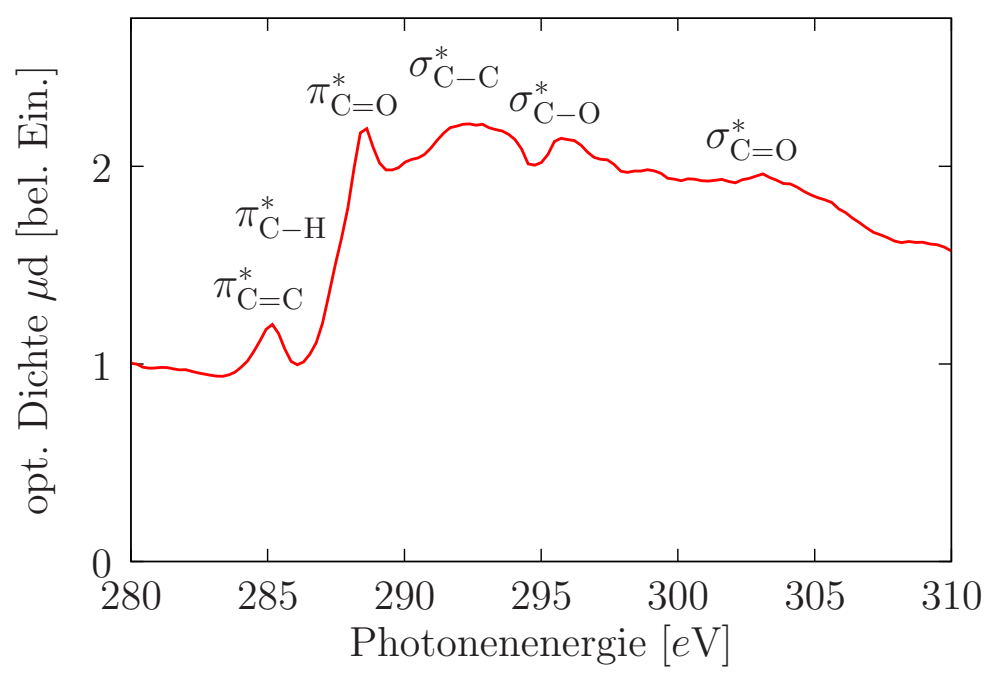

Abbildung 4.1: NEXAFS-Messung einer etwa $250 \mathrm{~nm}$ dicken PMMA-Schicht hergestellt mit 1.100 Pulsen bei $125 \mathrm{~mJ} \mathrm{~cm}^{-2}$.

Da die FT-IR-Spektroskopie im Falle der erwarteten $\mathrm{C}=\mathrm{C}$-Doppelbindungen also keine Ergebnisse lieferte, wurde zusätzlich die Röntgennahkantenabsorptionsspektroskopie (NEXAFS) angewandt. Durch NEXAFS können besonders die Doppelbindungen der Polymerschicht genauer untersucht werden. Oberhalb der Absorptionskante (etwa $287 \mathrm{eV}$ ) entsprechen die Spektren ziemlich gut denen aus der Literatur 99]. Zusätzlich ist bei $285 \mathrm{eV}$ sehr schön ein ausgeprägter Peak der $\pi_{\mathrm{C}=\mathrm{C}}^{*}$-Bindung zu erkennen, wie er für laserdeponiertes PMMA durch den vorherigen Inkubationsprozess zu erwarten ist (Abb. 4.1). Außerdem ist die Intensität der $\pi_{\mathrm{C}=\mathrm{O}}^{*}$-Bindung sehr viel geringer als durch die Literatur erwartet. Dies spricht für eine Verarmung des PMMA an Estergruppen. Diese werden durch den Depositionsprozess teilweise abgespalten.

Da die Inkubation eine Bildung von Doppelbindungen im Material beschreibt, müssen hierfür zunächst andere Bindungen aufgebrochen werden, um anschließend neue Bindungen zu ermöglichen. Durch diese Brechung von Bindungen erhält man eine Verkürzung der Ketten des Ausgangsmaterials. BlAnCHET et al. haben gezeigt, dass bereits 900 Pulse bei $39 \mathrm{~mJ} \mathrm{~cm}^{-2}$ ausreichen, um die Kettenlänge des Polymers von $500 \mathrm{~kg} \mathrm{~mol}^{-1}$ auf $8 \mathrm{~kg} \mathrm{~mol}^{-1}$ abzusenken [8]. Diese chemischen Änderungen des Polymers werden näher in Kapitel 4.4 beschrieben.

Durch die Bestrahlung und somit durch die Verkürzung der Ketten ist eine deutlich bessere Löslichkeit in Lösungsmitteln zu verzeichnen [94]. Gleichzeitig beschreiben KüPER und Stuke ein Absinken der Transmission des UV-Lichts bei $248 \mathrm{~nm}$ durch einen Beschuss mit 1.000 Pulsen bei $40 \mathrm{~mJ} \mathrm{~cm}^{-2}$ [58]. Die Transmission von infraroter Strahlung sinkt dabei auf nur noch $6 \%$ des Ausgangswertes. Durch diese Untersuchungen mittels Infrarotspektroskopie wurden die Veränderungen im Tar- 
getmaterial zum einen mit einer Veränderung der Ester-Seitengruppe und zum anderen mit einer Bildung von $\mathrm{C}=\mathrm{C}$-Doppelbindungen in der Hauptkette (backbone) beschrieben, was den Inkubationsprozess hier bestätigt.

Dass die Inkubation wirklich ein rein photochemischer und nicht ein photothermischer Prozess ist, bestätigen die Experimente von KüPER und STUKE, bei denen durch Bestrahlung mit einer Wellenlänge von $308 \mathrm{~nm}(4,0 \mathrm{eV}$, XeCl-Excimerlaser) keine Inkubation und kein Abtrag beobachtet wurde. Bestrahlt man aber zuvor das Material mit einer Wellenlänge von $248 \mathrm{~nm}$, was eine Inkubation bewirkt, so lässt sich danach das Material mit Laserpulsen von $308 \mathrm{~nm}$ Wellenlänge abtragen [58. Ein photochemischer Mechanismus mit mindestens einem Übergang ist somit denkbar, da die verwendeten $4,0 \mathrm{eV}$ ohne Verluste gerade ausreichen würden, um C-C-Bindungen aufzubrechen (s. Tab. 3.1). Für stärkere Einfachbindungen oder gar Doppelbindungen reicht diese Energie nicht zur Bindungsbrechung aus.

Durch Inkubation wird das Polymer also zunächst photochemisch verändert. Die Kettenlänge des Materials wird reduziert, wodurch in der Hauptkette die Bildung von Doppelbindungen (Chromophore) begünstigt wird. Hierdurch erhöht sich die Absorption im Material, was anschließend einen guten Abtrag des Materials erst ermöglicht.

Die Anzahl der Laserpulse, die für die Inkubation benötigt werden, also bis eine Materialdeposition mit guter Abtragsrate möglich ist, hängt stark von der verwendeten Laserfluenz ab [100] (s. Kap. 3.2). Mit steigender Fluenz nimmt die Anzahl der benötigten Pulse stark ab. Kurz oberhalb der Depositionsschwelle (bei etwa $130 \mathrm{~mJ} \mathrm{~cm}^{-2}$ ) werden über 300 Pulse benötigt, bis eine konstante Depositionsrate gewährleistet ist (Abb. 4.2). Bei hohen Fluenzen von mehreren $\mathrm{J} \mathrm{cm}^{-2}$ ist der Bereich der Inkubation nicht mehr klar abzugrenzen. Hier findet ein Materialabtrag ab dem ersten Puls statt. Die Depositionsrate dagegen steigt bis zu etwa 70 Pulsen an und ist erst danach konstant bei typischerweise $1 \mathrm{~nm} / \mathrm{Puls}$ [87].

Da die Absorption des Polymers direkt mit der für die Bestrahlung verwendeten Wellenlänge korreliert, findet man entsprechend für den Inkubationsprozess unterschiedliche Ausprägungen. So ist PMMA z. B. bei $193 \mathrm{~nm}$ ein sehr viel stärkerer Absorber als bei $248 \mathrm{~nm}$ [100]. SRINIVASAN et al. haben entsprechende Experimente bei $193 \mathrm{~nm}$ Wellenlänge $(6,4 \mathrm{eV})$ durchgeführt und beobachtet, dass nur 20-40 Pulse bei $160 \mathrm{~mJ} \mathrm{~cm}^{-2}$ ausreichen, um die Inkubationsphase abzuschließen [94].

Dass nach dem Inkubationsprozess an der Targetoberfläche eine konstante Depositionsrate folgt, lässt sich mit Hilfe des LAMBERT-BEERschen Gesetzes erklären (s. Formel 3.1). Die Energie, die die Targetoberfläche trifft, dringt in das Material ein und fällt dabei nach dem LAMBERT-BEERschen Gesetz exponentiell ab. Damit werden die obersten Schichten am stärksten verändert, was bei sehr hohen Fluenzen sogar schon zu einem Abtrag führt. Während der nächsten Pulse wird dann das Material entsprechend dem Materialabtrag weiter in die Tiefe verändert. Bei hohen Energiedichten ist dies im Targetquerschnitt bis zu $10 \mu \mathrm{m}$ Tiefe sichtbar (s. Abb. 3.8 b). Durch die so steigende Absorption kann die Laserstrahlung irgendwann 


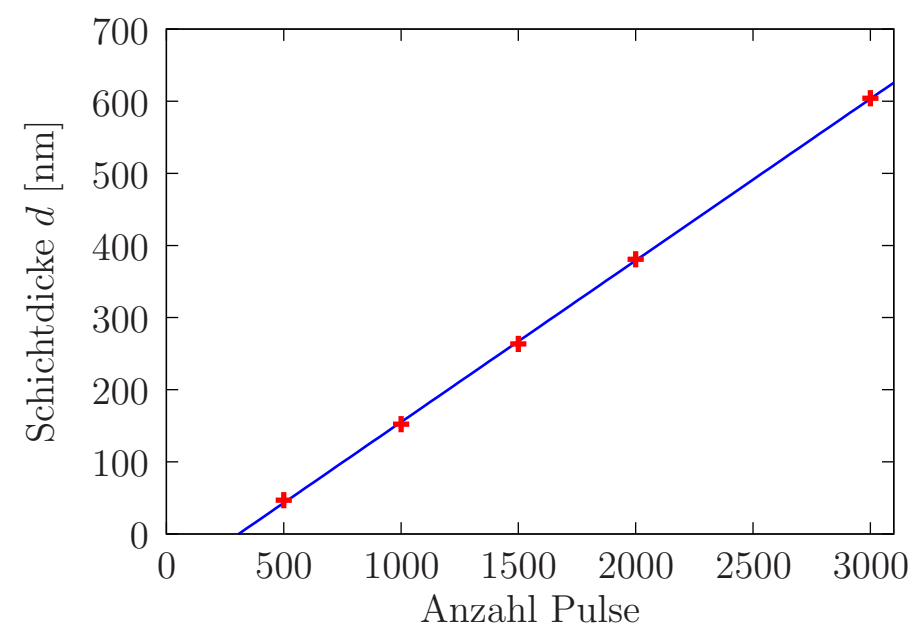

Abbildung 4.2: Inkubationseffekt bei einer verwendeten Laserenergiedichte von

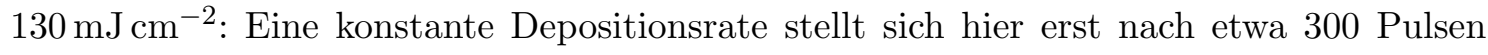
ein.

keine noch tieferen Bereiche im Target erreichen. An der Oberfläche wird Material abgetragen sobald die Absorption hoch genug ist. Somit tritt ein Gleichgewicht zwischen Inkubation und Abtrag ein. Wird an der Oberfläche Material abgetragen, kann das Target entsprechend in der Tiefe weiter inkubiert werden.

Hier wurde nun die Inkubation, die einer Schichtablation und -deposition vorausgeht, diskutiert. In Kapitel 3 wurde bereits der Laserfluenzbereich, in dem eine Schichtdeposition möglich ist, und die daraus resultierenden Morphologien der deponierten Schichten, nochmals vorgestellt. Im Folgenden sollen diese Morphologieuntersuchungen ausgeweitet werden.

\subsection{Schichtdickenabhängigkeit}

Als Erweiterung der Morphologieuntersuchungen aus Kapitel 3.3 soll hier auf die Schichtdickenabhängigkeit der Oberflächenmorphologie eingegangen werden. Deponiert man nur sehr wenig Material, erhält man zunächst keine geschlossenen Schichten; es bilden sich perkolierende Strukturen aus (Abb. $4.3 \mathrm{a}$ ). Die dort deponierte Materialmenge entspricht etwa $4 \mathrm{~nm}$. Das PMMA wächst also nicht sofort flach auf der Substratoberfläche, sondern hat eine leichte Tendenz zur Entnetzung. Dieses Verhalten ist auf dem Si-Substrat bei Raumtemperatur nur bei der dünnsten, hier deponierten Schicht zu sehen ist. Das Entnetzungsverhalten der deponierten PMMA Schichten verstärkt sich z. B. auf raueren Oberflächen [84], auf anderen Substraten wie Glas oder bei höheren Substrattemperaturen (s. Kap. 6.3.1).

Die etwa $50 \mathrm{~nm}$ hohen inselartigen Strukturen, die bei diesen Untersuchungen zu- 

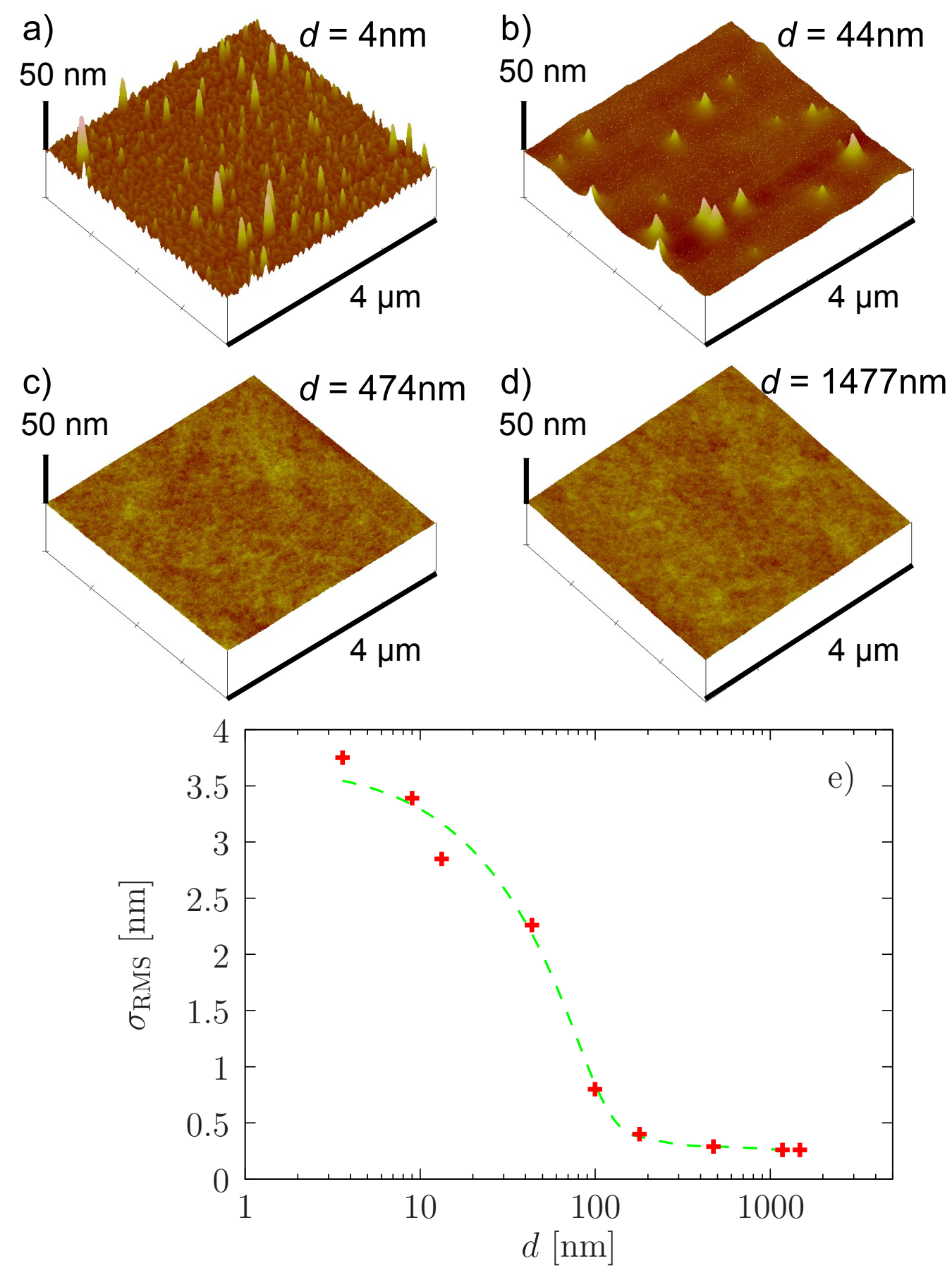

Abbildung 4.3: AFM-Bilder von PMMA-Schichten hergestellt bei $125 \mathrm{~mJ} \mathrm{~cm}^{-2}$ mit a) 15 Pulsen (RMS-Rauigkeit von $\left.\sigma_{\mathrm{RMS}}=3,8 \mathrm{~nm}\right)$, b) 150 Pulsen $\left(\sigma_{\mathrm{RMS}}=2,3 \mathrm{~nm}\right)$, c) 1.500 Pulsen $\left(\sigma_{\mathrm{RMS}}=0,3 \mathrm{~nm}\right)$, d) 5.000 Pulsen $\left(\sigma_{\mathrm{RMS}}=0,3 \mathrm{~nm}\right)$. Die sich ergebenden Rauigkeiten sind in e) gegen die Schichtdicke (aus Ellipsometermessungen) aufgetragen. 
sätzlich auf dem Substrat zu sehen sind (Abb. $4.3 \mathrm{a}, \mathrm{b})$, stammen nicht direkt vom Schichtwachstum. Es konnte nicht festgestellt werden, ob es sich um Verunreinigungen auf dem Substrat oder um Unebenheiten des Substrats selbst handelt. Bei der PLD von Metallen sind diese Substratstrukturen nie beobachtet worden. Eine Erklärung dafür ist, dass Metallatome bzw. -ionen solch eine hohe Energie beim Auftreffen auf das Substrat haben, dass die Substratoberfläche durch die Deposition nochmals gereinigt wird. Dies beinhaltet auch ein teilweises absputtern der natürlichen $\mathrm{SiO}_{2}$-Schicht auf dem Substrat. Der hohe Sputteryield bei der PLD wurde für einige Metalle von Sturm diskutiert [98]. Da für die Deposition der glatten PMMA-Schichten nur sehr geringe Laserfluenzen verwendet werden, scheinen hier die Molekülenergien nun nicht mehr auszureichen, um die Substratoberfläche zu beeinflussen. Es ist allerdings zu erkennen, dass die Unebenheiten - welcher Herkunft auch immer - keinen Einfluss auf unsere deponierte Schicht haben. Das PMMA legt sich einfach glatt auf bzw. zwischen die Strukturen (Abb. 4.3 b) und glättet diese bei ausreichender Schichtdicke vollständig aus (Abb. 4.3 c). Dies lässt sich sehr schön in der Auftragung der ermittelten RMS-Rauigkeiten aus den AFM-Bildern gegen die Schichtdicke aus Ellipsometriemessungen sehen (Abb. 4.3e). Die anfänglichen Rauigkeiten mit $\sigma_{\mathrm{RMS}}=3,5 \mathrm{~nm}$ werden von der deponierten PMMA-Schicht ab etwa $200 \mathrm{~nm}$ vollständig ausgeglättet. Die glatte PMMA-Schicht raut dabei auch mit steigender Schichtdicke nicht wieder auf (Abb. $4.3 \mathrm{~d}$ ), was einen konstanten Rauigkeitswert von etwa $\sigma_{\mathrm{RMS}}=0,3 \mathrm{~nm}$ ergibt. Diese PMMA-Schichten wurden auch schon zur Diskussion von Glättungsmechanismen bei RöDER beschrieben [84]. Dort zeigte sich auch, dass sich der Entnetzungsmechanismus durch rauere Substrate verstärkt. Dies wurde auch schon von REHSE für Polystyrol (PS) beschrieben [82].

Im Gegensatz zu dieser Arbeit spielte bei der Bachelorarbeit von EBERL die Entnetzung eine wichtige Rolle bei der Laserdeposition von PEMA-Schichten [24]. Selbst sehr dicke Schichten im $\mu$ m-Bereich entnetzten von den Si-Substraten, wodurch z. B. die Bestimmung von Depositionsraten oder Aussagen über die Dauer des Inkubationsprozesses, wie sie hier für PMMA in Kapitel 4.1 gemacht wurden, erschwert wurden. Die Entnetzung der PEMA-Schichten von den Si-Substraten ist viel stärker im Vergleich zu den laserdeponierten PMMA-Schichten, da das PEMAAusgangsmaterial eine viel geringere Glasübergangstemperatur von nur $T_{g}=65^{\circ} \mathrm{C}$ (Angabe des Herstellers ALDRICH) aufweist. Dadurch ist bei Raumtemperatur die Mobilität der PEMA-Ketten schon sehr viel größer als beim PMMA und die Entnetzung kann leichter in größeren Bereichen stattfinden. Die Glasübergangstemperatur wird außerdem durch den Depositionsprozess beeinflusst. Wie sich $T_{g}$ vom Ausgangsmaterial zur laserdeponierten Schicht im Fall von PMMA ändert, wird später in Kapitel 5.1 diskutiert.

Neben oberflächlichen Veränderungen mit Änderung der Schichtdicke wurde von BAKER für sehr dünne PMMA-Schichten bis zu $2 \mathrm{~nm}$ mit zunehmender Schichtdicke auch ein leichter Abfall des Brechungsindex von $n=1,498$ auf $n=1,488$ beschrieben [5]. Aus Ellipsometriemessungen lässt sich neben den Schichtdicken auch der jewei- 


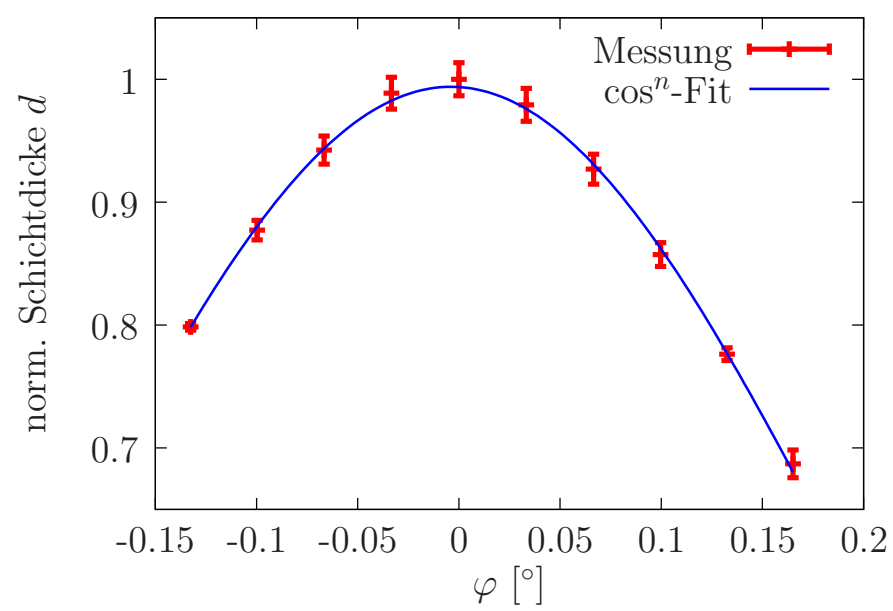

Abbildung 4.4: Schichtdickenverteilung aus Ellipsometermessungen: Die Probe wurde mit 500 Pulsen bei $125 \mathrm{~mJ} \mathrm{~cm}{ }^{-2}$ hergestellt. Aus dem $\cos ^{n} \varphi$-Fit ergibt sich hier $n=28$.

lige Brechungsindex $n$ der PMMA-Schichten bestimmen. Allerdings konnte hier die Abhängigkeit des Brechungsindex von der Schichtdicke nicht festgestellt werden, da bei sehr geringen Schichtdicken durch die eben beschriebenen Anfangsrauigkeiten die Fehler der Ergebnisse zu groß sind. Für größere Schichtdicken von mehreren hundert Nanometern liegen die Brechungsindizes hier mit $n \approx 1,5$ in der gleichen Größenordnung wie in der Literatur beschrieben.

Hier konnte also gezeigt werden, ab welcher Schichtdicke sich die PMMA-Schichten glatt deponieren lassen bzw. dass es möglich ist, Strukturen auf der Substratoberfläche durch die Deposition von PMMA zu glätten. Die bei dieser Deposition entstehende Verteilung der Schichtdicke soll nun diskutiert werden.

\subsection{Schichtdickenverteilung}

Da Schichten, die bei Laserfluenzen kurz oberhalb der Depositionsschwelle hergestellt wurden, sehr glatt sind, lassen sich Schichtdicken mit geringem Fehler aus Ellipsometriemessungen gewinnen. Man muss also nicht wie in Kapitel 3.4 für die Schichtdickenmessung der vernetzten Komponente der Tröpfchenschichten auf XRRMessungen zurückgreifen. Die sich aus Ellipsometriemessungen ergebende Schichtdickenverteilung einer glatten PMMA-Schicht ist in Abbildung 4.4 zu sehen. Der $\cos ^{n} \varphi$-Fit (s. Kap. 3.4) ergibt hier für die glatten Schichten eine starke Bündelung mit $n=28$. Damit entspricht die Bündelung in etwa auch der gerichteten Deposition, wie sie für Metalle vorkommt. Dies ist eine sehr viel schmalere Dickenverteilung der Polymerschicht als für hohe eingestrahlte Laserfluenzen (s. Kap. 3.4). Der Grund der stärkeren Bündelung ergibt sich daraus, dass für die Deposition bei den gerin- 


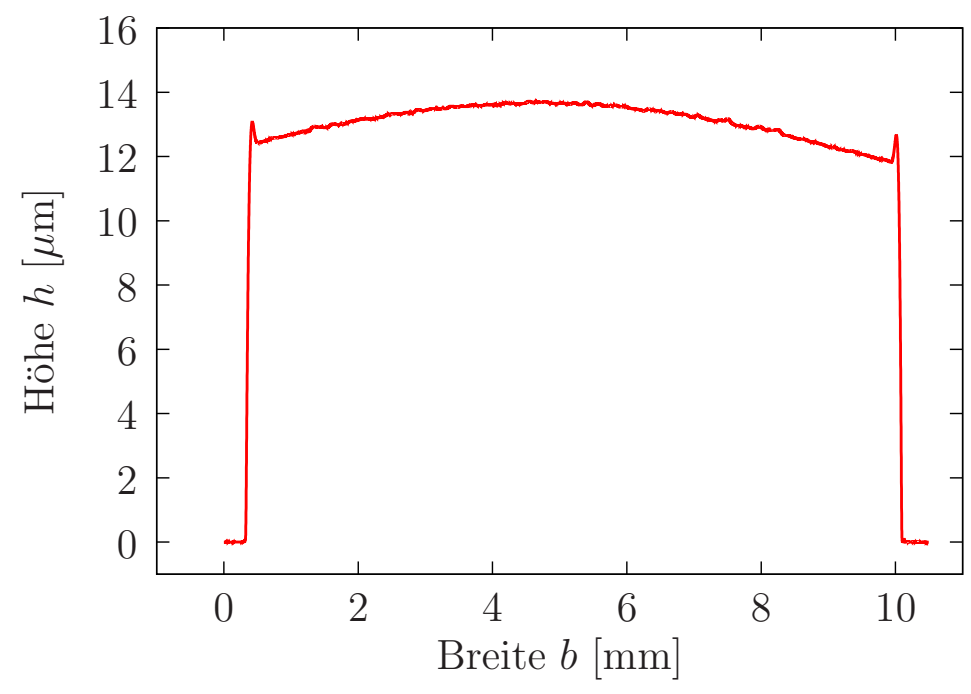

Abbildung 4.5: Schichtdickenverteilung aus Profilometermessungen: Profil einer $1 \mathrm{~cm}$ breiten PMMA-Schicht mit 100.000 Pulsen bei $125 \mathrm{~mJ} \mathrm{~cm}^{-2}$ hergestellt. Es wird dabei eine maximale Schichtdicke von etwa $13,5 \mu \mathrm{m}$ erreicht.

gen Laserfluenzen der Brennfleck auf der Targetoberfläche von $0,02 \mathrm{~cm}^{2}$ auf $0,8 \mathrm{~cm}^{2}$ aufgezogen wurde. Somit wird die Bündelung des ablatierten Materials wieder entsprechend verstärkt.

Um einen besseren Überblick über die Homogenität der deponierten Schicht zu bekommen, wurde ein Profil der Probe erstellt (Abb. 4.5). Um den Vergleich zur Substratoberfläche zu haben, wurden die Substrate an den Seiten eingeklemmt und danach die Schicht deponiert. Nach der Schichtdeposition wurden die Substrate wieder aus der Klemmung gelöst, wodurch dann an den Rändern das blanke Si-Substrat zu erkennen ist. Abgesehen von den leichten Hügeln an den Schichträndern, die durch die begünstigte Oberflächenenergie durch die Anlagerung an die Klemmbacken entstanden sind, sieht man die glatte Schicht mit dem eben beschriebenen Schichtdickenmaximum in der Substratmitte. Überprüft man hier die Schichtdickenverteilung wieder durch einen $\cos ^{n} \varphi$-Fit, ergibt sich eine Verteilung mit $n=30$. Die Stärke der Bündelung bleibt also so gut wie unbeeinflusst von der betrachteten lateralen Ausdehnung der Schicht oder von der deponierten Menge des Materials.

Für diese Arbeit stellt solch ein Schichtdickengradient keine Schwierigkeit dar, da für die meisten Messmethoden hauptsächlich eine große Materialmenge wichtig ist. In der Bachelorarbeit von EBERL war der Schichtdickengradient sogar ausdrücklich erwünscht, um an einer deponierten Schicht das Verhalten für verschiedene Schichtdicken untersuchen zu können [24]. Wäre das Ziel, möglichst homogene Schichtdicken zu deponieren, ließe sich das z. B. einfach durch eine geeignete Verschiebung des rotierenden Substrats gegen den Schwerpunkt der Plasmakeule, also gegen das 


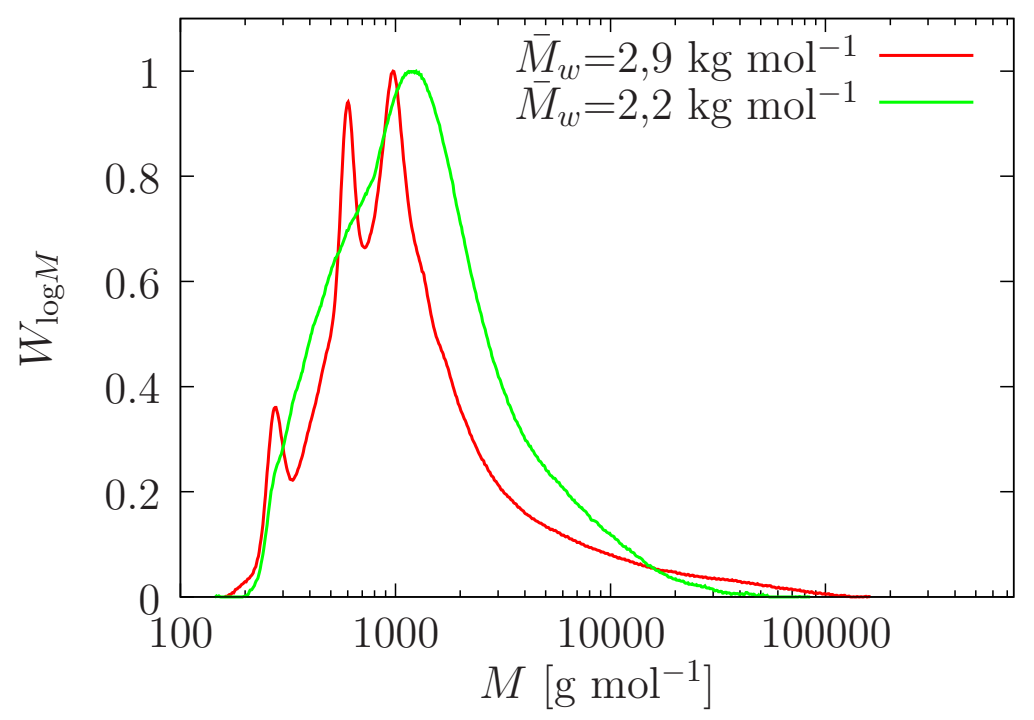

Abbildung 4.6: Beispielhaft sind zwei SEC-Messungen von PMMA-Schichten dargestellt, die bei $130 \mathrm{~mJ} \mathrm{~cm}^{-2}$ hergestellt wurden. Trotz gleicher Herstellungsbedingungen ergeben sich aus den Messungen mittlere Molmassen von $\bar{M}_{w}=2.900 \mathrm{~g} \mathrm{~mol}^{-1} \mathrm{bzw} . \bar{M}_{w}=$ $2.200 \mathrm{~g} \mathrm{~mol}^{-1}$ mit Polydispersitäten von $D=3,5$ bzw. 2,3.

Maximum der deponierten Schichtdicke, realisieren [72]. Dies hätte allerdings einen höheren Verlust der ablatierten Materialmenge zufolge, da sich mehr Material an dem Substrat vorbei bewegt. Deshalb wurde auf diese Art der Schichtoptimierung im Rahmen dieser Arbeit verzichtet. Im Folgenden sollen die Schichten näher auf ihre chemischen und mechanischen Eigenschaften untersucht werden, für deren Untersuchung besonders hohe Materialmengen bzw. Schichtdicken benötigt werden.

\subsection{Chemische Eigenschaften}

Durch die bereits diskutierte Inkubation des PMMA-Ausgangsmaterials zu Beginn des Depositionsprozesses wird ersichtlich, dass dies große Auswirkungen auf die chemischen Eigenschaften der deponierten Schichten wie z. B. die Kettenlänge hat.

Das PMMA-Ausgangsmaterial besitzt laut Hersteller eine mittlere Molmasse von $\bar{M}_{w}=996.000 \mathrm{~g} \mathrm{~mol}^{-1}$. Dieser Wert wurde von ALDRICH durch GrößenausschlussChromatographie (Size Exclusion Chromatography, SEC) bestimmt. Solche Messungen wurden hier ebenfalls durchgeführt, um Änderungen der Kettenlängen durch die Schichtdeposition feststellen zu können. Der Vergleich des Ausgangspulvers mit dem gepressten PMMA-Target ergab, dass durch den Pressvorgang bei erhöhten Temperaturen die Molmasse in keinster Weise beeinflusst wird.

Untersucht man nun Schichten, die bei Raumtemperatur und Laserfluenzen von 
$130 \mathrm{~mJ} \mathrm{~cm}^{-2}$ hergestellt wurden, stellt man eine starke Verringerung der mittleren Molmasse auf etwa $\bar{M}_{w}=(2,2-2,9) \mathrm{kg} \mathrm{mol}^{-1}$ fest (Abb. 4.6). Durch die logarithmische Auftragung werden hier vor allem die kleinsten Molmassen hervorgehoben, die der Größenordnung der kleinsten Oligomere des PMMA entsprechen, allerdings zur Berechnung der mittleren Molmasse kaum ins Gewicht fallen. Aus den Messungen lässt sich außerdem die Polydispersität des Polymers bestimmen. Dies ist das Verhältnis von Gewichtsmittel $M_{w}$ zu Zahlenmittel $M_{n}$, was ein Maß für die Verteilung der Molmassen beschreibt. Eine höhere Polydispersität

$$
D=\frac{M_{w}}{M_{n}}
$$

entspricht somit einer breiteren Verteilung. Glatte Schichten, wie sie eben beschrieben wurden, ergeben Polydispersitäten von $D=2,3-3,5$. Das Ausgangsmaterial bzw. das verwendete PMMA-Target zeigen Polydispersitäten von $D=3,4$. Da die Ergebnisse aus den SEC-Messungen stark von der jeweiligen Kalibrierung bzw. vom verwendeten Eichstandard abhängen und bei kleineren Molmassen außerdem einen größeren Messfehler aufweisen, weichen hier die Ergebnisse für PMMA-Schichten, die unter gleichen Bedingungen hergestellt wurden, voneinander ab. So ist in Abbildung 4.6 zu erkennen, dass in einer Messung die Oligomerpeaks besser voneinander getrennt auftreten, das andere Mal ergibt sich ein gemeinsames Maximum, einzelne Peaks sind nicht aufösbar. In den späteren Kapiteln werden deshalb zum Vergleich dieser Schichten mit modifizierten Proben immer Messungen aus demselben Messdurchgang verwendet. Damit wird sichergestellt, dass die Messfehler minimiert werden und so aus den SEC-Messungen klare Tendenzen zwischen verschieden gearteten Schichten erkennbar sind.

Durch eine hochaufgelöste Massenfeinanalyse konnten des Weiteren deponierte PMMA-Schichten im Bereich der kleinen Oligomere zwischen $100 \mathrm{u}$ und $2.000 \mathrm{u}$ genauer untersucht werden. Die Messung einer unter Normalbedingungen deponierten Schicht ergab in diesem Messbereich eine breite Massenverteilung. Die PMMAKetten liegen in der deponierten Schicht also in allen Längen und Modifikationen, d. h. unter anderem mit abgespaltener Seitengruppe vor. Ein Ausschnitt dieser Messung ist in Abbildung 4.7 gezeigt. Gut zu erkennen ist dabei zum Einen das Vorkommen der Molekülmassen in einfach ionisiertem Zustand, was durch die vergleichsweise hohe Intensität zu erkennen ist. Zum anderen kommen auch mehrfache Ionisierungen zustande, wobei die Massen mit entsprechend geringerer Intensität detektiert werden. Die intensiveren Peaks lassen sich Abständen von $100 \mathrm{u}$ zuordnen. Dieser feste Abstand lässt sich über das ganze Spektrum wiederfinden und ist mit Pfeilen einer Farbe gekennzeichnet. Verschoben zu einem Peaksatz kommen weitere Peaks mit Abständen von $100 \mathrm{u}$ zueinander vor. Jeder Satz ist durch Pfeile einer Farbe gekennzeichnet. Der Abstand der Peaks von $100 \mathrm{u}$ entspricht dabei einer Monomereinheit MMA. Das die Kettenlänge aber nicht genau einem Vielfachen des MMA - die intensiven Peaks liegen nicht genau bei $400 \mathrm{u}, 500 \mathrm{u}$, etc. - entspricht, könnte 


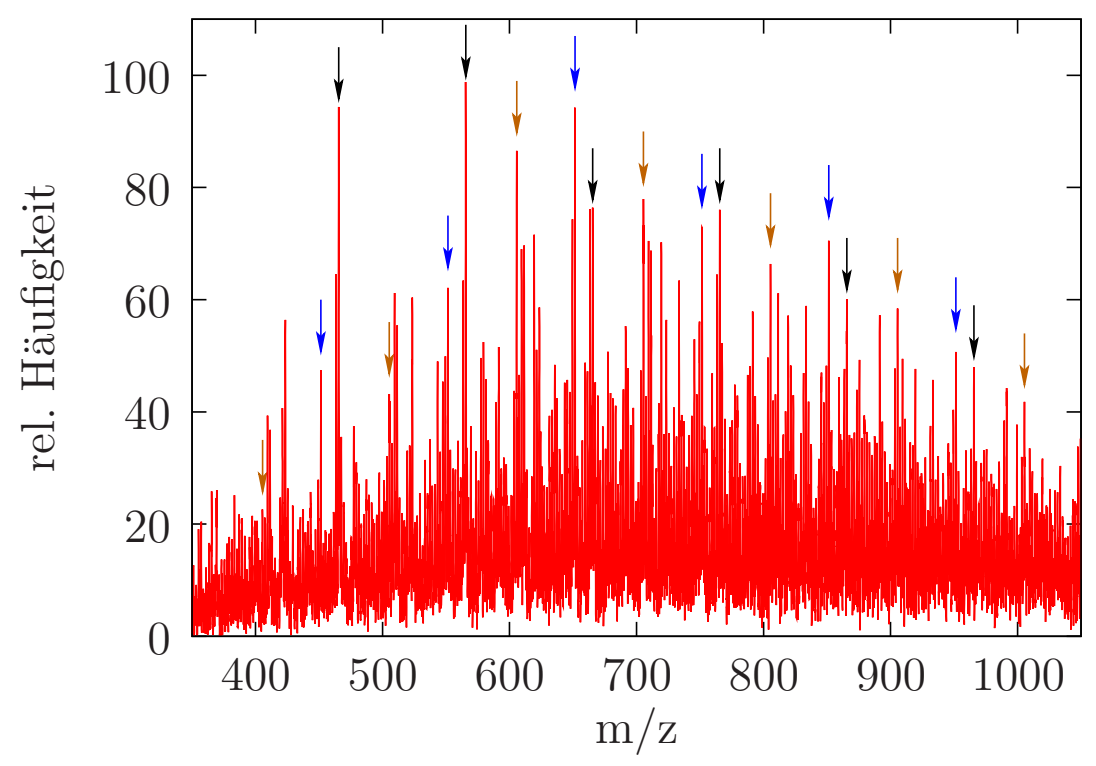

Abbildung 4.7: In die hochaufgelöste Massenfeinanalyse sind Pfeile in verschiedenen Farben eingetragen. Pfeile der gleichen Farbe haben jeweils einen Abstand von $100 \mathrm{u}$ und zeigen somit, dass intensivere Peaks in Abständen der Monomereinheit MMA vorkommen, aber nicht insgesamt einem Vielfachen des MMA entsprechen.

z. B. am Verlust von Seitengruppen oder anderen Fragmenten durch den Depositionsprozess liegen.

Zum Vergleich ergaben SEC-Messungen von Schichten, die bei hohen Laserfluenzen hergestellt wurden, dass die tröpfchenförmige Komponente gegenüber dem Ausgangsmaterial eine reduzierte mittlere Molmasse von $\bar{M}_{w}=5.800 \mathrm{~g} \mathrm{~mol}^{-1}$ besitzt [102]. Diese Tröpfchen, die nicht nur auf der Schicht, sondern auch teilweise in die Schicht eingebaut und von der glatten Komponente bedeckt sind, lassen sich in Tetrahydrofuran (THF) lösen, während der glatte Schichtanteil vollständig erhalten bleibt. Die Schichtrückstände auf dem Substrat ließen blasenartige Hüllen zurück. SüsKE beschrieb diesen Rückstand dadurch, dass in THF nur unvernetztes Polymer löslich ist; der glatte Schichtanteil ist demnach stark vernetzt, während die Tröpfchen aus größtenteils unvernetztem intakten Polymer bestehen [101]. Da also nur eine Komponente dieser Schichten vermessen werden konnte, ergab sich so auch ein sehr geringer Wert für die Polydispersität von $D=1,5$.

Es konnte hier also gezeigt werden, dass bei der Laserdeposition von glatten PMMA-Schichten Kettenspaltungen und Abspaltungen von Seitengruppen vorkommen. Diese Spaltungen sind zwingend notwendig, um die freien Bindungspartner zu erzeugen, die für die $\mathrm{C}=\mathrm{C}$-Doppelbindungsbildung während der Inkubation benötigt werden. Welche Auswirkungen diese chemischen Veränderungen auf die mechanischen Eigenschaften haben, soll nun gezeigt werden. 

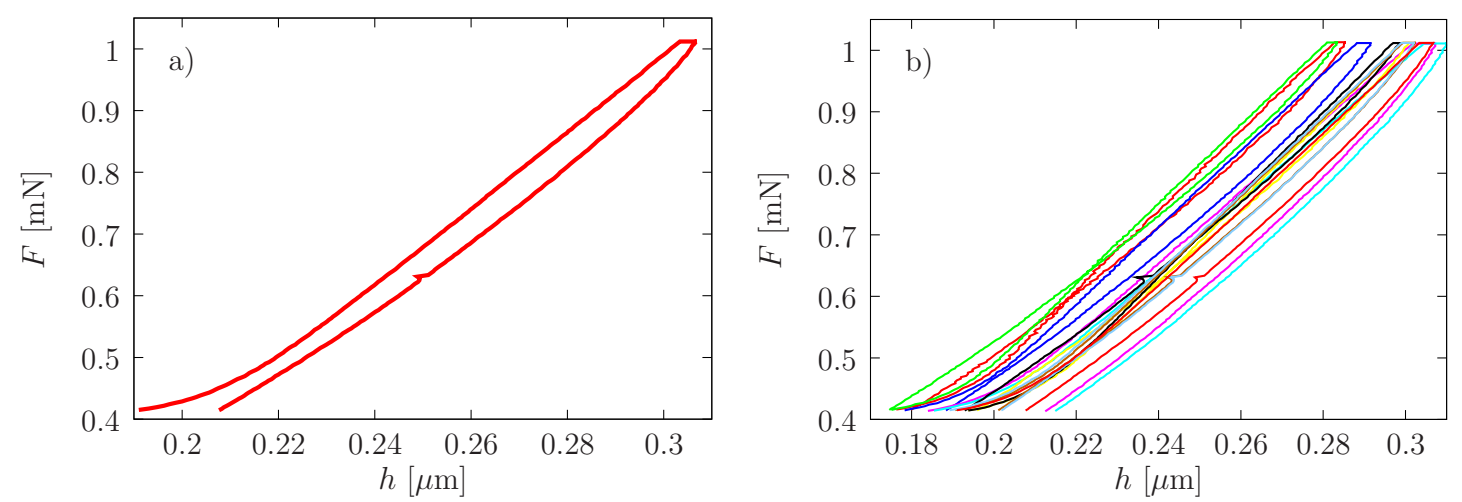

Abbildung 4.8: Kraft-Eindringtiefe-Messung eines PMMA-Targets: Gezeigt ist hier a) der typische Kurvenverlauf für solch ein elastisches Material und b) die Reproduzierbarkeit, ein Maß für die Homogenität. Es ergibt sich aus den Messungen des Targets als Ausgangsmaterial eine Universalhärte von $H U=(290 \pm 25) \mathrm{N} \mathrm{mm}^{-2}$.

\subsection{Mechanische Eigenschaften}

Für eine praktische Anwendung der deponierten Polymerschichten sind vor allem die mechanischen Eigenschaften von großer Bedeutung. Um genauere Aussagen über die laserdeponierten Filme machen zu können, wurden hier Härteuntersuchungen durchgeführt. Dafür wurde für die deponierten Schichten die Universalhärte, wie in Kapitel 2.4.1 beschrieben, bestimmt. Um die Ergebnisse miteinander vergleichen zu können, wurden die Messparameter fest gelassen. Hier wurde immer eine maximale Kraft von $F_{\max }=1 \mathrm{mN}$ in $20 \mathrm{~s}$ angefahren. Durch Messung und Vergleich der Hinund Rückkurve lassen sich Aussagen über die Plastizität bzw. Elastizität der Probe machen.

Die verwendeten PMMA-Targets zeigen ein fast vollständig elastisches Verhalten, was daraus zu erkennen ist, das Hin- und Rückrichtung der Kraft-EindringtiefeKurve fast übereinander liegen (Abb. 4.8a). Die Homogenität der Probe lässt sich über die Reproduzierbarkeit der Messkurven feststellen, wobei sich für das Target eine Abweichung der Messwerte von etwa 9\% ergibt (Abb. 4.8b). Damit ist das gepresste Target als homogen anzusehen. Aus diesen Messungen ergibt sich eine Universalhärte von $H U=(290 \pm 25) \mathrm{N} \mathrm{mm}^{-2}$. Die hohe Elastizität ergibt sich beim Ausgangsmaterial aus den sehr großen Kettenlängen, wodurch die Polymerketten mit sich selbst und miteinander sehr verschlauft sind. Dadurch wird die Beweglichkeit der Ketten herabgesetzt und sie bewegen sich wie ein Gummi bei der Entlastung der Indentorspitze wieder in ihren ursprünglichen Zustand zurück. Die Härtewerte selbst hängen natürlich auch von der Art der Targetherstellung ab, wobei die PMMA-Targets hier aber sehr reproduzierbar gepresst wurden.

Für alle im Rahmen dieser Arbeit durchgeführten Härtemessungen an PMMA- 

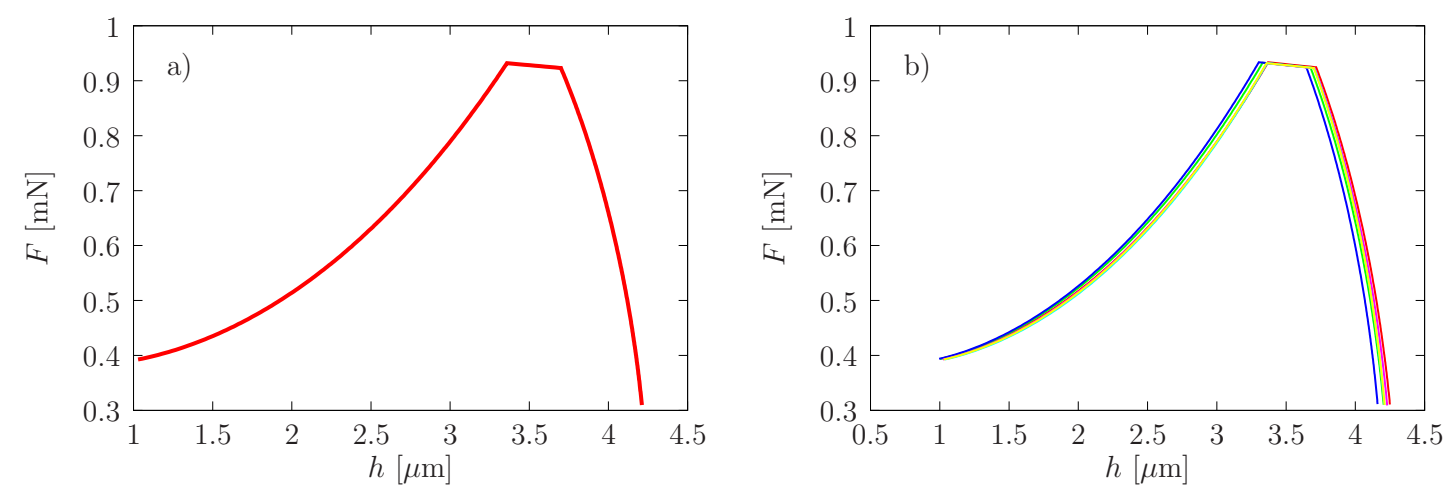

Abbildung 4.9: Kraft-Eindringtiefe-Messung einer glatten PMMA-Schicht hergestellt mit 100.000 Pulsen bei $125 \mathrm{~mJ} \mathrm{~cm}^{-2}$ : Gezeigt ist hier a) der typische Kurvenverlauf für diese Schichten, die ein sehr plastisches Verhalten beschreiben und b) die gute Reproduzierbarkeit, ein Maß dafür, dass die Schichten sehr homogen sind. Es ergibt sich aus den Messungen für diese Schicht eine Universalhärte von $H U=(2,8 \pm 0,2) \mathrm{N} \mathrm{mm}^{-2}$.

Schichten konnte der Einfluss des Si-Substrats - auch bei höheren Eindringtiefen - ausgeschlossen werden, da die Messkurvenform gleichmäßig und ohne plötzlichen Knick verläuft. Die verwendeten Si-Substrate selbst haben eine Härte von $H U=8.000 \mathrm{~N} \mathrm{~mm}^{-2}$ und zeigen einen Kraft-Eindringtiefe-Verlauf, der sich durch viele Stufen auszeichnet.

Bei glatten PMMA-Schichten, die mit maximal $125 \mathrm{~mJ} \mathrm{~cm}^{-2}$ hergestellt wurden, erkennt man ein vollständig plastisches Verhalten (Abb. 4.9a). Die Schichten geben sogar so leicht nach, dass trotz der Reduzierung der Kraft der Indentor noch tiefer in die Schicht einsinkt, d. h. das Kriechen ist schneller als die Kraftreduktion. Dieses Verhalten zeigt sich homogen über die ganze Probe verteilt mit einer Abweichung der Messwerte von $7 \%$ (Abb.4.9b). Die glatten Schichten haben eine Universalhärte von $H U=(2,8 \pm 0,2) \mathrm{N} \mathrm{mm}^{-2}$. Dass die Schichten nun sehr viel plastischer sind als das Ausgangsmaterial, erklärt sich direkt aus den SEC-Messungen, aus denen bereits eine starke Verkürzung der mittleren Kettenlänge deutlich wurde. Somit ist die Verschlaufung der Ketten miteinander reduziert und die kürzeren Ketten und Fragmente können mit dem Indentor plastisch gegeneinander verschoben werden.

Um zu überprüfen, wie stabil diese hergestellten Schichten sind und welche Faktoren die Härte beeinflussen, wurde das Langzeitverhalten der Härte untersucht (Abb. 4.10). Die Schichten bleiben mehr als zwei Wochen lang stabil; danach steigt die Universalhärte an. Dieser Anstieg ist - gegenüber dunkel gelagerten Schichten bei den Schichten, die im Exsikkator gelagert wurden, etwas höher. Dabei kann es für das unterschiedliche Härtungsverhalten verschiedene Ursachen geben. Die Schichten, die im Dunkeln lagerten, sollten vor jeglicher Nachbestrahlung durch UV-Licht geschützt sein. Diese Schichten wurden nur während der Messzeiten Licht ausgesetzt, 


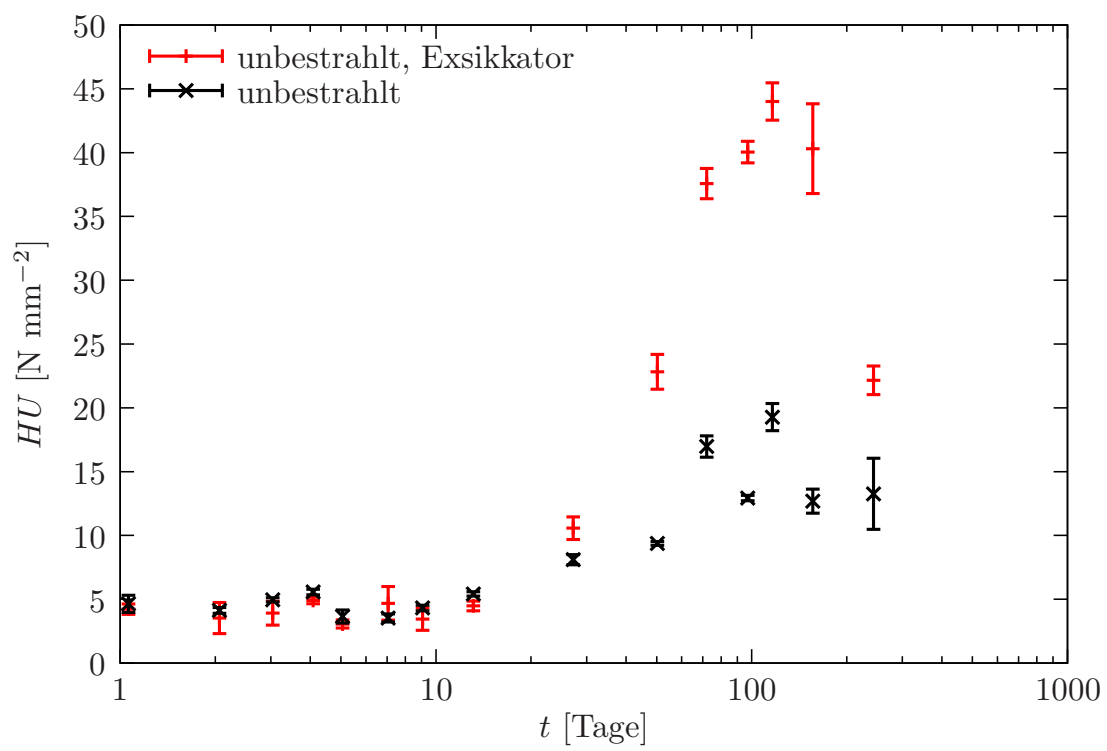

Abbildung 4.10: Langzeitverhalten glatter PMMA-Schichten zum Vergleich unter Normalbedingungen und im Exsikkator gelagert. Die Härteänderung wurde hier über viele Monate verfolgt.

trotzdem härteten sie etwas nach. Die im Exsikkator gelagerten Schichten wurden durch das Glas evtl. leichter UV-Strahlung ausgesetzt. Andererseits lag das etwas stärkere Nachhärten auch an der mit Hilfe von Trockenperlen geschaffenen Umgebung im Exsikkator. Trotz nicht eindeutig geklärter Ursache für die unterschiedliche Nachhärtung wurden aufgrund dieser Messungen nun alle laserdeponierten PMMASchichten nicht mehr im Exsikkator gelagert.

Zum Vergleich mit den eben gezeigten glatten PMMA-Schichten und um zu zeigen, dass die erreichte Homogenität dieser glatten PMMA-Schichten wirklich ein Novum ist, sind hier auch noch Kraft-Eindringtiefe-Kurven für eine Tröpfchenschicht aufgenommen worden. Aufgrund des hohen Anteils der Tröpfchenkomponente mit etwas höherer mittlerer Molmasse als bei den glatten Schichten und aufgrund der Vernetzung der hier glatten Komponente sind diese Schichten weniger plastisch als die eben beschriebenen Schichten, aber durch ihre trotzdem hohe Reduktion der Ausgangskettenlänge bei weitem nicht mehr so elastisch wie das PMMA-Target selbst (Abb. 4.11a). Durch die Tröpfchen, die in allen Größen vorkommen und statistisch über die ganze Probe verteilt liegen, sind diese zweikomponentigen Schichten sehr inhomogen, wodurch die Messwerte hier einen Fehler von $35 \%$ aufweisen (Abb. $4.11 \mathrm{~b}$ ). Die Tröpfchenschichten haben so eine Universalhärte von $H U=(74 \pm 26) \mathrm{N} \mathrm{mm}^{-2}$.

Da die geringe Rauigkeit der hier deponierten PMMA-Schichten für praktische Anwendungen sehr interessant ist, wurden diese z. B. verwendet, um Polymer/MetallSchichtpakete herzustellen. Ein Querschnitt eines solchen Schichtpakets aus fünf 

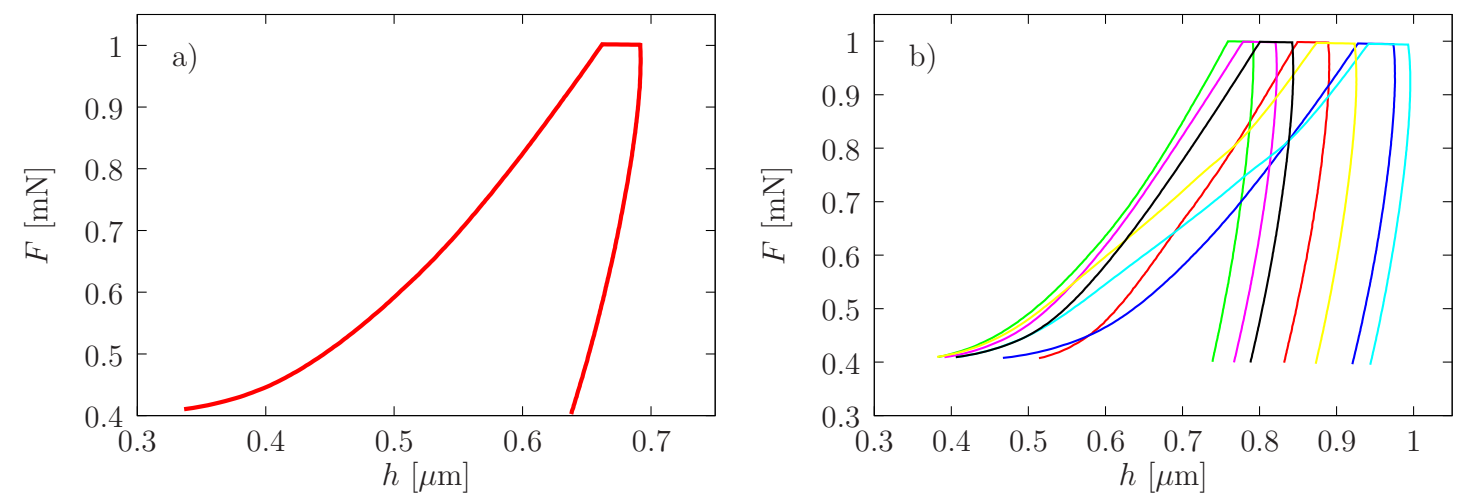

Abbildung 4.11: Kraft-Eindringtiefe-Messung einer PMMA-Schicht mit Tröpfchen hergestellt mit 20.000 Pulsen bei $4,5 \mathrm{~J} \mathrm{~cm}^{-2}$ : Gezeigt ist hier a) der typische Kurvenverlauf für diese Schichten, die ein etwas elastischeres Verhalten zeigen als die glatten Schichten und b) die große Streuung der Einzelmessungen, ein Maß dafür, dass die Schichten sehr inhomogen sind. Es ergibt sich aus den Messungen für diese Schicht eine Universalhärte von $H U=(74 \pm 26) \mathrm{N} \mathrm{mm}^{-2}$.

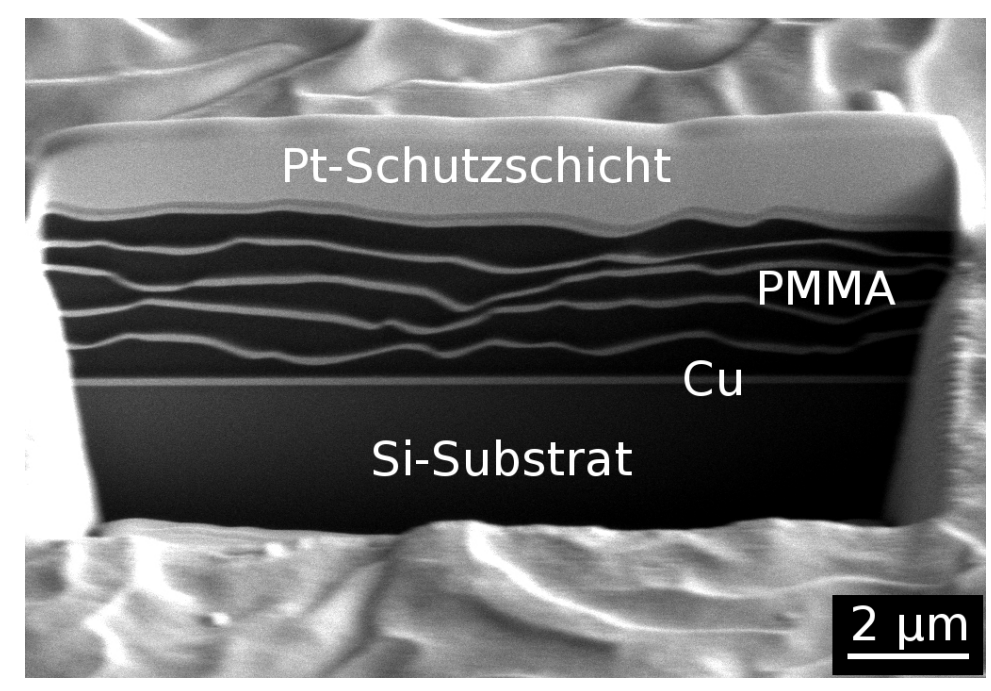

Abbildung 4.12: Querschnitt eines Schichtpakets aus fünf Cu/PMMA-Doppelschichten mit $\mathrm{Cu}$-Deckschicht. Deponiert wurden jeweils 30.000 Pulse Kupfer bei $7,6 \mathrm{~J} \mathrm{~cm}^{-2}(\widehat{=} 100 \mathrm{~nm})$

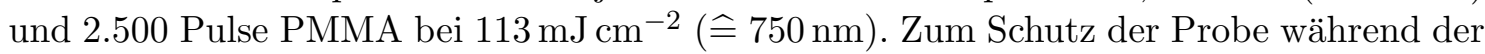
Querschnittpräparation wurde eine Pt-Schutzschicht aufgebracht. 
Doppellagen $\mathrm{Cu}$ und dem hier beschriebenen glatten PMMA mit $\mathrm{Cu}$-Deckschicht ist in Abbildung 4.12 zu sehen. Man erhält nicht wie erwartet ein glattes Schichtpaket, sondern erkennt eine starke Wellenbildung. Dies rührt von den starken Verspannungen im deponierten Kupfer her, die durch shot peening während des Depositionsprozesses bedingt sind [86]. Diese Verspannungen in den $\mathrm{Cu}$-Schichten können sich nun durch eine Verlagerung und damit durch Auswölbungen in die sehr weichen PMMA-Schichten abbauen.

\subsection{Fazit}

Bisher wurde die Herstellung von glatten PMMA-Schichten bei geringen Laserfluenzen vorgestellt und deren Eigenschaften im Vergleich mit dem Targetmaterial und den aus vorherigen Arbeiten bekannten Tröpfchenschichten diskutiert. Es zeigte sich, dass der Deposition von glatten PMMA-Schichten bei sehr geringen Laserfluenzen ein ausgeprägter Inkubationsprozess voran geht. Die daraufhin deponierte glatte Schicht ist in der Lage anfängliche Rauigkeiten zu glätten und raut auch mit steigender Schichtdicke nicht wieder auf. Die Schichtdickenverteilung zeigt durch den vergrößerten Brennfleck auf der Targetoberfläche für die deponierten glatten Schichten ähnliches Verhalten wie bei deponierten Metallschichten, also einen starken Schichtdickengradienten. Außerdem weisen die glatten Schichten durch den Depositionsprozess eine starke Reduzierung der mittleren Molmasse auf, wobei keine diskreten Zustände sondern vielmehr eine breite statistische Verteilung aller Molmassen zu verzeichnen war. Dies wird durch den Depositionsprozess bzw. schon durch den Inkubationsprozess erklärt. Zu sehen war eine Verringerung der $\mathrm{C}=\mathrm{O}$-Doppelbindungen, was auf eine Abspaltung von ganzen oder Teilen von Estergruppen hinweist. Außerdem wurden die durch Inkubation entstandenen $\mathrm{C}=\mathrm{C}$-Doppelbindungen nachgewiesen. Dass die glatten Schichten aufgrund des Depositionsprozesses nicht vernetzt wurden, zeigte sich nicht nur durch die Möglichkeit, die Schichten für die SECMessungen in THF zu lösen. Sie wiesen außerdem eine extrem geringe Universalhärte auf.

Im Folgenden soll nun auf die thermische Stabilität der PMMA-Schichten eingegangen werden, da die hier gezeigte Kettenlängenänderung auch andere Modifikationen wie etwa eine Absenkung der Glasübergangstemperatur zur Folge haben sollte (Kap. 5).

Außerdem ist die extrem geringe Härte der an sich glatt deponierten PMMASchichten wenig praktikabel und erschwert damit viele Anwendungen. Deshalb soll in dieser Arbeit weiterhin untersucht werden, inwiefern sich glatte PMMA-Schichten mit Laserfluenzen im Bereich der Depositionsschwelle herstellen lassen, die zusätzlich eine höhere Härte aufweisen (Kap. 6). 


\section{Thermische Behandlung der PMMA-Schichten}

In diesem Kapitel soll die Veränderung der laserdeponierten PMMA-Schichten durch thermische Behandlung bis hin zu deren vollständiger Zersetzung beschrieben werden. Bei thermischen Betrachtungen spielt die Glasübergangstemperatur $T_{g}$ des Materials eine wesentliche Rolle, weshalb zuerst darauf näher eingegangen werden soll (Kap. 5.1). Anschließend soll die thermische Stabilität des Schichtmaterials im Vergleich zum PMMA-Ausgangsmaterial untersucht werden (Kap. 5.2). Außerdem hat eine thermische Behandlung der Schichten auch Einfluss auf die chemischen Eigenschaften wie die Kettenlänge (Kap. 5.3). Eine Analyse der abdampfenden Moleküle soll das Verständnis der Zusammensetzung der laserdeponierten PMMA-Schichten fördern (Kap. 5.4).

\subsection{Bestimmung der Glasübergangstemperatur}

Allgemein lässt sich ein Glas definieren als „,amorpher Festkörper, der einen Glasübergang zeigt" [25]. Ein Glas ist amorph (gr.: ohne Gestalt), weist also ein Fehlen der Fernordnung auf, und ist somit vergleichbar mit einer erstarrten Flüssigkeit. Der Glasübergang lässt sich über den Vorgang des Abkühlens eines Glasbildners aus der Schmelze beschreiben. Die Glasübergangstemperatur $T_{g}$ entspricht der Temperatur, bei der ein Glas die größte Verformungsfähigkeit aufweist. Getrennt wird damit der spröde energieelastische Bereich eines Materials für $T<T_{g}$ (Glasbereich) vom weichen entropieelastischen Bereich für $T>T_{g}$ (gummielastischer bzw. viskoelastischer Bereich). Hierbei ist zu beachten, dass $T_{g}$ für ein Material nicht eindeutig festgelegt ist, sondern sich abhängig von verschiedenen Parametern verschieben kann [2, 39].

Im Falle der polymeren Glasbildner, zu denen das PMMA gehört, müssen für eine makroskopische Verformung ganze Ketten oder Kettensegmente bewegt werden, die durch kovalente Bindungen (starke Bindungen) zusammengehalten werden. Die schwachen Bindungen wie die VAN DER WAALS-Wechselwirkungen zwischen einzelnen Polymerketten lassen sich schon durch geringe Energiezufuhr leicht aufbrechen. Unterhalb der Glasübergangstemperatur werden (z. B. durch Verformung) nur lokale kollektive Prozesse mit wenigen beteiligten Segmenten aktiviert. Die auf diese Weise verursachte Bewegung, die die Verlagerung des Schwerpunkts eines Kettensegments zur Folge hat, wird als Rotation beschrieben, bei der mehrere $\mathrm{C}-\mathrm{C}$-Bindungen 


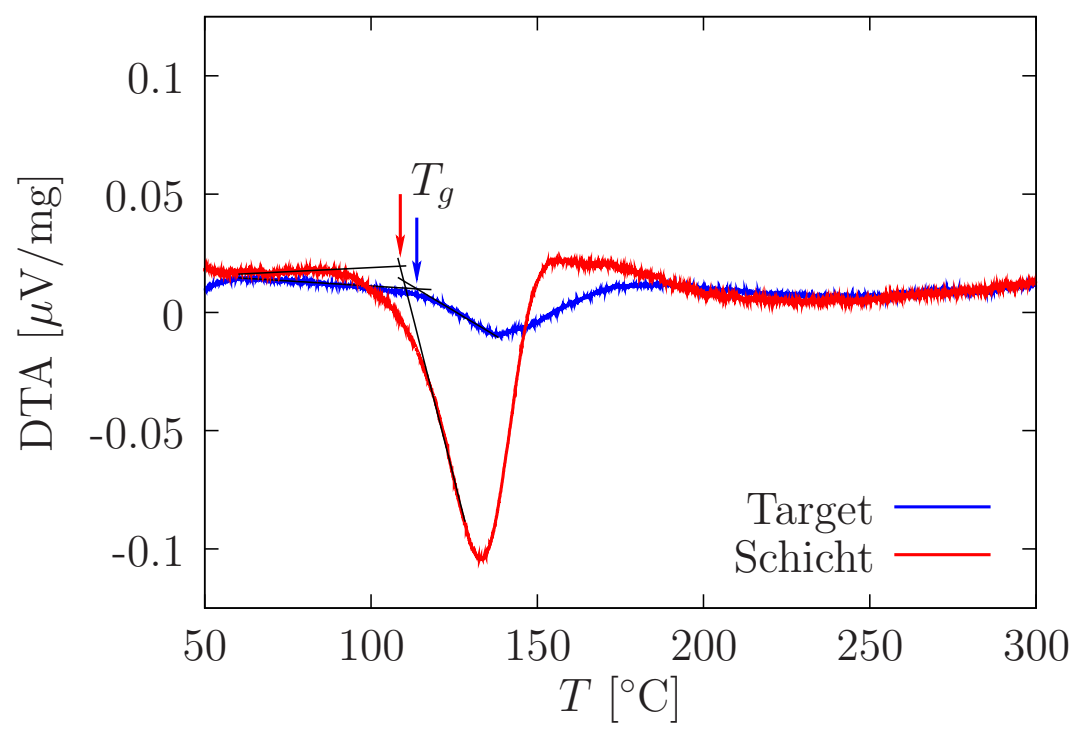

Abbildung 5.1: Differential-Thermoanalyse (DTA) mit einer Heizrate von $1 \mathrm{~K} / \mathrm{min}$; die Glasübergangstemperatur einer deponierten PMMA-Schicht liegt etwa bei $T_{g}=109^{\circ} \mathrm{C}$, die eines verwendeten PMMA-Targets etwa bei $T_{g}=114^{\circ} \mathrm{C}$. Die Ausgangsmasse für beide Messungen wurde mit etwa $35 \mathrm{mg}$ eingewogen.

beteiligt sind [110]. Betrachtet man dagegen eine Bewegung oberhalb der Glasübergangstemperatur, wird diese über Verschlaufungen beschrieben, die temporäre Verhakungspunkte verschiedener Ketten darstellen. Durch diese wird die freie Bewegung der Kettensegmente behindert. Die Bewegung einer Kette verläuft nach dem Reptationsmodell von DE GENNES in einem Kanal aus umgebenden Molekülen [18]. Es ergibt sich so ein viskoelastisches Verhalten bzw. bei noch höheren Temperaturen ein viskoses Fließen des Polymers. Bei einer Vernetzung des Polymers werden einzelne Ketten zusätzlich über kovalente Bindungen miteinander verbunden. Durch diese festen Vernetzungspunkte wird ein (rein) viskoses Verhalten auch bei höheren Temperaturen unterdrückt. Bei einem sehr stark vernetzten Polymer lässt sich überhaupt kein Glasübergang mehr nachweisen. Der Grenzfall totaler Vernetzung entspricht also dem Übergang von einem Polymer zu einem normalen Festkörper [40].

Die Glasübergangstemperatur ist keine materialspezifische Temperatur, sondern ist von Parametern abhängig wie z. B. der verwendeten Heizrate bei der Messung [40], der Kettenlänge des Polymers [77] oder der Grenzflächenenergie und Schichtdicke [30]. Ein Anstieg der Glasübergangstemperatur durch den Einfluss der Schichtdicke wurde für PMMA aber erst unterhalb von $40 \mathrm{~nm}$ gefunden [29]. Hier ist aufgrund des Ablationsprozesses bei der PLD vor allem eine Änderung der Glasübergangstemperatur durch eine Verkürzung der Kettenlänge zu erwarten. Die Glasübergangstemperatur $T_{g}$ des Schicht- und Ausgangsmaterials wurde hier mit Hilfe der 


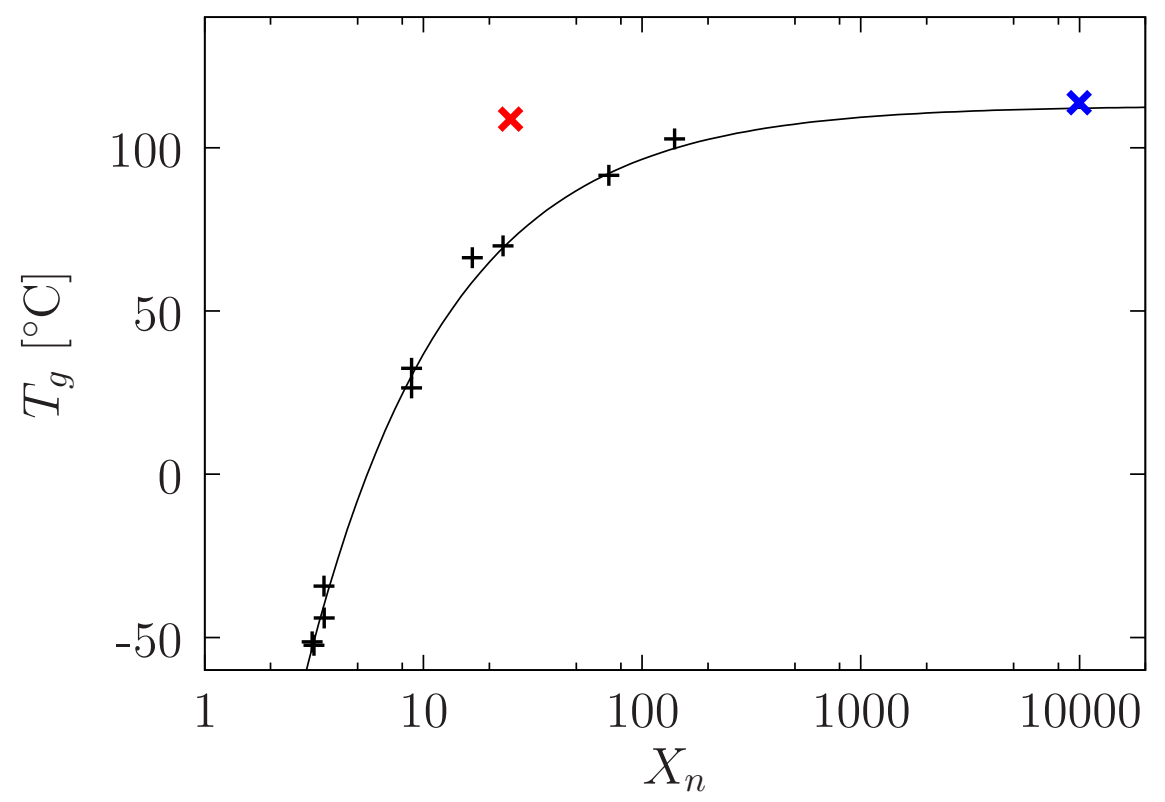

Abbildung 5.2: Abhängigkeit der Glasübergangstemperatur von der Kettenlänge für PMMA nach O'Driscoll [77] (schwarz). Zusätzlich eingezeichnet sind die von uns ermittelten Werte für Schicht (rot) und Target (blau).

Differential-Thermoanalyse (DTA) bestimmt. Verwendet wurde eine Heizrate von $1 \mathrm{~K} / \mathrm{min}$ und eine Ausgangsmasse von jeweils etwa $35 \mathrm{mg}$. Da sowohl die Schicht als auch das Ausgangsmaterial vor den Messungen in Tetrahydrofuran (THF) gelöst und in Messtiegeln wieder eingetrocknet wurde, ist ein Unterschied der Messergebnisse durch Einflüsse wie Schichtdicke oder Grenzflächenenergien hier auszuschließen. Die Analyse zeigt, dass oberhalb von $100^{\circ} \mathrm{C}$ ein endothermer Vorgang zu beobachten ist (Abb. 5.1). Die Glasübergangstemperatur entspricht dem onset des endothermen Peaks [12]. Vor allem für die DTA-Messung des Schichtmaterials setzt der endotherme Vorgang nur langsam ein. Zur Bestimmung der Glasübergangstemperatur wird deshalb der Schnittpunkt der Tangente an den ersten Wendepunkt mit der Verlängerung der Grundlinie abgelesen [76]. Für die PMMA-Schicht ist der Glasübergang (nach der Schnittpunktbestimmung über die Tangenten) bei etwa $T_{g}=109^{\circ} \mathrm{C}$ zu erkennen. Für das Ausgangsmaterial liegt der Übergang etwas höher bei etwa $T_{g}=114{ }^{\circ} \mathrm{C}$. Damit weicht dieser Wert etwas von dem bei ALDRICH mit $T_{g}=105^{\circ} \mathrm{C}$ angegebenen Wert ab. Alle Werte liegen aber in dem weiten Temperaturbereich, der für den Glasübergang in der Literatur für PMMA zu finden ist [13, 30, 55].

Die Kettenlängenabhängigkeit der Glasübergangstemperatur des Polymers ergibt sich nach O'DRISCOLL aus [77]:

$$
T_{g}=T_{g, \infty}-K / X_{n}^{2 / 3}
$$


mit mittlerer Kettenlänge $X_{n}$, polymerspezifischer Konstante $K$ und asymptotischem Wert $T_{g, \infty}$, zu dem die Glasübergangstemperatur $T_{g}$ bei höheren Kettenlängen tendiert. O'Driscoll gab für PMMA $X_{n}$ in Anzahl der Monomereinheiten, $K=353$ und $T_{g, \infty}=386$ an. Der von uns ermittelte Glasübergang des Targetmaterials $\left(X_{n}=9.960\right)$ passt dabei gut zum Grenzwert $T_{g, \infty}=113{ }^{\circ} \mathrm{C}$ der Vergleichskurve (Abb. 5.2). Der Glasübergang für die PMMA-Schichten dagegen liegt im Vergleich zu den Werten von O'Driscoll höher. Der Grund für diese Abweichung liegt zum Einen wohl an der Messmethode der DTA selbst, da auch das Targetmaterial seinen Glasübergang $9{ }^{\circ} \mathrm{C}$ über dem vom Hersteller angegebenen Wert zeigt, zum Anderen wird die hier angegebene Glasübergangstemperatur des Schichtmaterials nur einer mittleren Kettenlänge zugeordnet, da das Schichtmaterial eine breite Verteilung verschieden langer Ketten aufweist (s. Kap. 4.4). Entscheidend ist aber wohl vor allem, dass das PMMA selbst durch z. B. Doppelbindungsbildung und Abspaltung verschiedener Fragmente leicht verändert wurde. Eine höhere Glasübergangstemperatur kommt dabei wohl hauptsächlich durch das Abspalten von Estergruppe zustande. Da in der Reihe der Poly(alkyl methacrylate) die Glastemperatur mit zunehmender Länge der Estergruppe stark gesenkt wird [37, 21], sollte ein Abspalten einer Vielzahl von Seitengruppen den gegenteiligen Effekt auf das so modifizierte PMMA haben. Außerdem verschiebt sich die mittlere Molmasse bei thermischer Behandlung der Schichten zunächst zu größeren Werten, wodurch sich der Messpunkt der Literaturkurve annähern würde. Diese chemischen Veränderungen werden in Kapitel 5.3 genauer diskutiert. Zunächst soll näher auf den Massenverlust durch thermische Behandlung der Proben bzw. auf deren thermische Stabilität eingegangen werden.

\subsection{Thermische Stabilität}

Nachdem die für thermische Untersuchungen wichtige Glasübergangstemperatur diskutiert und die Ergebnisse des laserdeponierten PMMA mit denen des Ausgangsmaterials verglichen wurden, sollen nun Aussagen zur thermischen Stabilität der Proben gemacht werden. Hierfür wurde der Massenverlust von Schicht- und Targetmaterial in Abhängigkeit von der Temperatur gemessen.

Für die Thermogravimetrische Analyse (TGA) einer PMMA-Schicht und eines PMMA-Targets wurde eine Heizrate von $1 \mathrm{~K} / \mathrm{min}$ verwendet. Es wurden jeweils Massen von etwa $35 \mathrm{mg}$ in die Messtiegel eingewogen, was den $100 \%$ zu Beginn der Messungen entspricht. Eine Schicht weist nach einer Auslagerung bis $450^{\circ} \mathrm{C}$ noch etwa $9 \%$ seiner Ausgangsmasse auf. Dieser immense Massenverlust verläuft stetig mit steigender Temperatur, aber mit zwei sehr deutlich zu erkennenden Stufen (Abb. 5.3). Unterhalb der Glastemperatur von $109^{\circ} \mathrm{C}$ ist lediglich eine Massenabnahme von $2 \%$ zu verzeichnen. Die Targetmessung weist unterhalb der Glasübergangstemperatur ebenfalls einen Masseverlust von $2 \%$ auf, was zeigt, dass die Schichten bei Temperaturen unterhalb $T_{g}$ ähnlich stabil sind wie das Ausgangsmaterial. Die Schicht verliert 


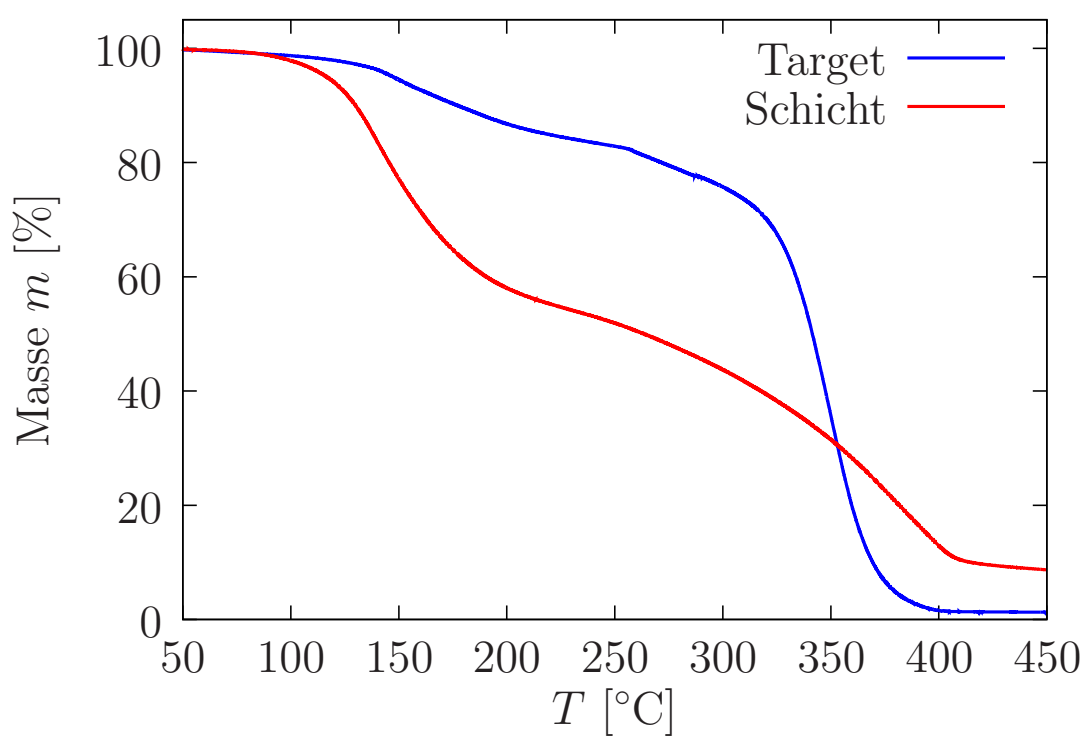

Abbildung 5.3: Thermogravimetrische Analyse (TGA) einer PMMA-Schicht und eines PMMA-Targets mit einer Heizrate von $1 \mathrm{~K} / \mathrm{min}$; $100 \%$ entsprechen hier jeweils einer eingewogenen Masse von etwa $35 \mathrm{mg}$.

bis zum Ende der ersten Stufe unterhalb von $200{ }^{\circ} \mathrm{C}$ etwa $40 \%$ seiner Masse verloren. Das Targetmaterial zeigt dabei einen weniger ausgeprägten zweistufigen Masseverlust, sondern verliert den Hauptteil seiner Masse erst oberhalb einer Temperatur von etwa $330{ }^{\circ} \mathrm{C}$ und ist bei $400{ }^{\circ} \mathrm{C}$ bereits vollständig abgedampft. Die zurückbleibende Masse bei der Messung des Schichtmaterials spricht für einen vernetzten und damit schwer löslichen Rückstand, der aufgrund seiner schwarzen Färbung wahrscheinlich stark kohlenstoffhaltig ist. Das dieser Massenverlust gleichzeitig zu einer chemischen Änderung des noch vorhandenen Materials führt, soll nun diskutiert werden.

\subsection{Chemische Eigenschaften}

Durch die Laserdeposition der PMMA-Schichten bei höheren Temperaturen ist zunächst ein Anwachsen der mittleren Molmasse zu bemerken. So steigt sie hier von $\bar{M}_{w}=2.900 \mathrm{~g} \mathrm{~mol}^{-1}$ für eine Schicht, die bei Raumtemperatur hergestellt wurde, auf $\bar{M}_{w}=7.400 \mathrm{~g} \mathrm{~mol}^{-1}$ für eine Schicht, die bei $200^{\circ} \mathrm{C}$ hergestellt wurde (Abb. 5.4). Durch die SEC-Messung ist zu erkennen, dass nicht ausschließlich ein Abdampfen kurzer Ketten zu einem Anstieg der mittleren Molmasse führt, sondern dass sich ein weiteres Maximum bei höheren Molmassen ausbildet. Es ergibt sich also durch das Abdampfen der Seitenketten bzw. von Kettenbruchstücken die Möglichkeit zur Quervernetzung der Ketten, weshalb höhere Molmassen messbar sind. Besonders stark ist damit auch die Vergrößerung der Verteilung der Molmassen also die Er- 


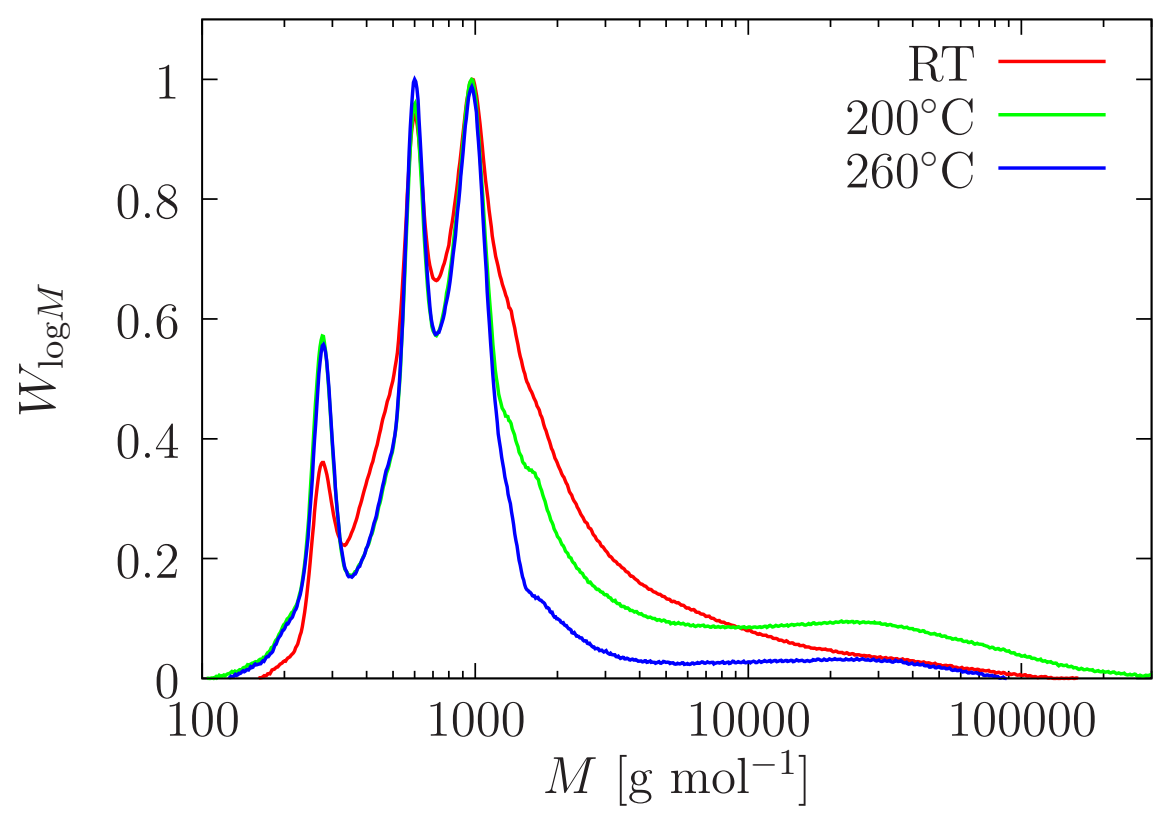

Abbildung 5.4: SEC-Messungen von PMMA-Schichten hergestellt bei $125 \mathrm{~mJ} \mathrm{~cm}^{-2}$ bei Raumtemperatur (RT), $200^{\circ} \mathrm{C}$ und $260^{\circ} \mathrm{C}$. Die mittlere Molmasse der PMMA-Schichten steigt von $\bar{M}_{w}=2.900 \mathrm{~g} \mathrm{~mol}^{-1}$ (Polydispersität $\left.D=3,5\right)$ bei der Herstellung bei RT an auf $\bar{M}_{w}=7.400 \mathrm{~g} \mathrm{~mol}^{-1}(D=9,5)$ bei $200^{\circ} \mathrm{C}$ und sinkt auf $\bar{M}_{w}=2.200 \mathrm{~g} \mathrm{~mol}^{-1}(D=3,5)$ für die Herstellung bei $260^{\circ} \mathrm{C}$. 


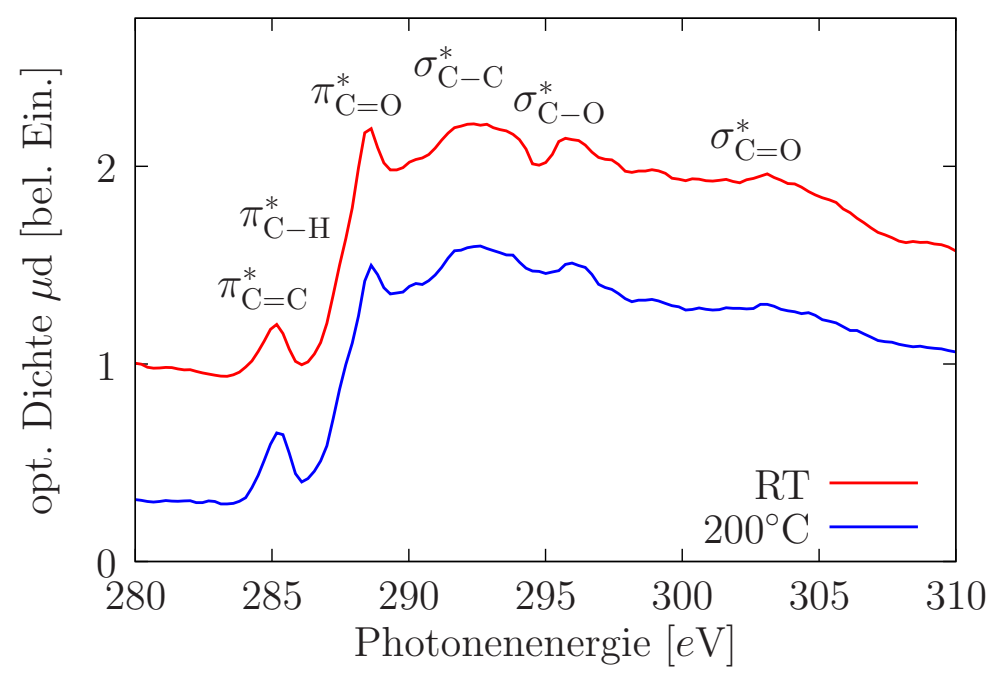

Abbildung 5.5: NEXAFS-Messungen einer bei Raumtemperatur mit 1.100 Pulsen bei $125 \mathrm{~mJ} \mathrm{~cm}^{-2}$ hergestellten etwa $250 \mathrm{~nm}$ dicken PMMA-Schicht und einer entsprechenden bei Raumtemperatur mit 2.100 Pulsen hergestellten und nachträglich bei $200^{\circ} \mathrm{C}$ ausgelagerten Schicht.

höhung der Polydisperität von $D=3,5$ auf $D=9,5$. Durch diese Zunahme der mittleren Molmasse mit der Temperatur ist auch ersichtlich, dass die in Abbildung 5.2 eingetragene mittlere Molmasse für eine bei Raumtemperatur deponierte Schicht zu klein für die gemessene Glasübergangstemperatur ist, da sich die Kettenlänge mit zunehmender Temperatur verschiebt. Würde man also den Messpunkt zu höheren Kettenlängen hin verschieben, käme man der Literaturkurve sehr viel näher. Erhöht man die Temperatur bei der Deposition weiter, ist in der SEC-Messung sichtbar, dass man in den Temperaturbereich der Zersetzung des PMMA kommt. Bei einer Temperatur von $260^{\circ} \mathrm{C}$ bei der Herstellung liegt die mittlere Molmasse nur noch bei $\bar{M}_{w}=2.200 \mathrm{~g} \mathrm{~mol}^{-1}$, die Polydispersität wieder bei $D=3,5$. Die durch erhöhte Temperaturen und der daraus resultierenden Zersetzung entstehenden Schichtfragmente werden in Kapitel 5.4 beschrieben.

Betrachtet man nicht die gesamten Ketten, sondern vergleicht nur die Doppelbindungen, lassen sich die thermisch gefundenen Prozesse sehr gut bestätigen. Vergleicht man z. B. die NEXAFS-Messungen einer RT-Schicht mit einer Schicht, die bei $200^{\circ} \mathrm{C}$ hergestellt wurde, ist durch den thermischen Einfluss eine Abnahme der $\pi_{\mathrm{C}=\mathrm{O}^{-}}^{*}$ Bindung und in gleichem Maße eine Zunahme der $\pi_{\mathrm{C}=\mathrm{C}^{*}}^{*}$ Bindung zu verzeichnen. Eine Verringerung der $\mathrm{C}=\mathrm{O}$-Doppelbindung spricht dabei eindeutig für die $\mathrm{Ab}-$ spaltung der Estergruppe. Die Bildung der $\mathrm{C}=\mathrm{C}$-Doppelbindungen wurde im PMMA überhaupt erst durch die Verkürzung der langen Polymerketten während des Inkubationsprozesses ermöglicht (s. Kap. 4.1 und 4.4). Durch weitere Abspaltungen von z. B. Seitengruppen wird nun neben der Quervernetzung des Materials ebenfalls die 


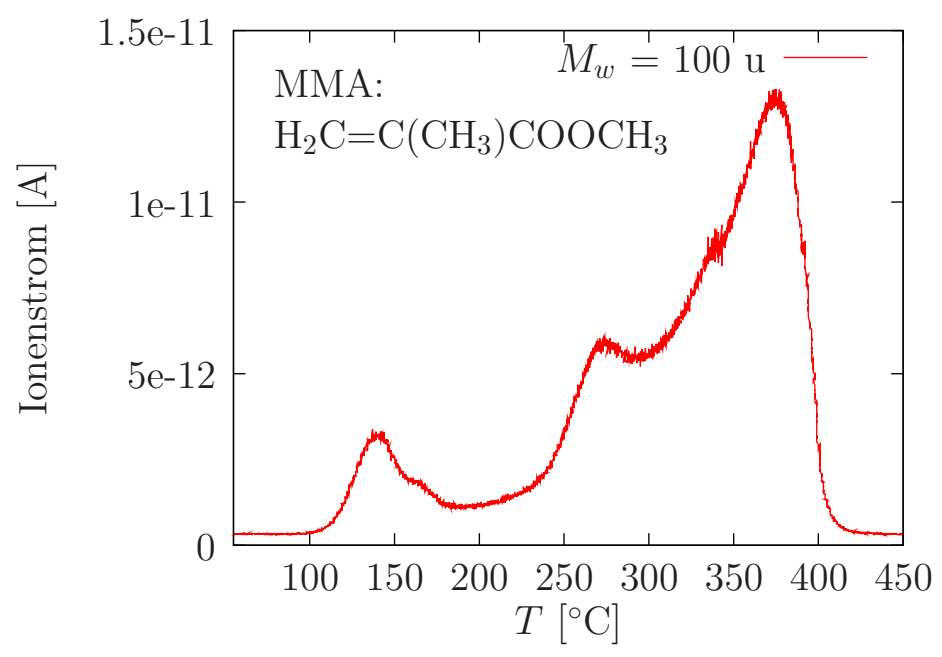

Abbildung 5.6: Massenspektrometrie-Messung bei $100 \mathrm{u}$. Dies entspricht dem Monomer Methyl methacrylat (MMA).

Bildung von $\mathrm{C}=\mathrm{C}$-Doppelbindungen weiter gefördert. Wie die abgespaltenen und abgedampften Fragmente genau zusammengesetzt sind, soll nun diskutiert werden.

\subsection{Analyse der Schichtfragmente}

Um die Vorgänge im Probenmaterial während einer thermischen Behandlung besser verstehen zu können, betrachtet man sich die dabei abdampfenden Bestandteile. Deshalb wurde zur Beschreibung der Zusammensetzung der laserdeponierten PMMA-Schichten eine massenspekrometrische Analyse der durch thermische Behandlung abdampfenden Bestandteile durchgeführt.

Mit Hilfe der Massenspektrometrie wurde der Ionenstrom für abdampfende Massen bis zu einer maximalen Molmasse von $100 \mathrm{u}$ erfasst, wobei der Temperaturbereich von etwa Raumtemperatur bis $450^{\circ} \mathrm{C}$ entsprechend den DTA- und TGAMessungen erfasst wurde. Es lässt sich somit maximal das Monomer, also das Methyl methacrylat (MMA) aufösen (Abb. 5.6).

Die zu detektierenden Molmassen wurden nach den zu erwartenden abdampfenden Molekülen nach BLANCHET und GuPTA [7, 41] ausgewählt und aus allen Einzelspektren die jeweiligen Maxima im Ionenstrom abhängig von der Temperatur ausgelesen. Eine Auflistung der Hauptpeakpositionen für alle detektierten Molmassen und die entsprechende Zusammensetzung der Moleküle ist in Tabelle 5.1 zu sehen. Die Auftragung dieser stärksten Peaks aus den jeweiligen Einzelmessungen (Abb. 5.7) zeigt eine bimodale Verteilung, die mit den beiden Stufen der Massenverlustmessung in Abbildung 5.3 sehr gut übereinstimmt. Kleinere Peaks bzw. Schultern wurden da- 


\begin{tabular}{|c|c|c|c|}
\hline \multirow{2}{*}{$\begin{array}{r}\text { Molmasse } \\
{[\mathrm{g} / \mathrm{mol}]}\end{array}$} & \multicolumn{2}{|c|}{ Peakposition } & \multirow[t]{2}{*}{ Zusammensetzung } \\
\hline & $T\left[{ }^{\circ} \mathrm{C}\right]$ & Ionenstrom $[\mathrm{A}]$ & \\
\hline \multirow[t]{2}{*}{2} & 134 & $5,0 \cdot 10^{-10}$ & $\mathrm{H}_{2}$ \\
\hline & 406 & $3,6 \cdot 10^{-10}$ & \\
\hline \multirow[t]{2}{*}{15} & 144 & $1,2 \cdot 10^{-10}$ & $\mathrm{CH}_{3}$ \\
\hline & 385 & $2,9 \cdot 10^{-10}$ & \\
\hline \multirow[t]{2}{*}{16} & 137 & $6,6 \cdot 10^{-11}$ & $\mathrm{CH}_{4}, \mathrm{O}$ \\
\hline & 403 & $1,7 \cdot 10^{-10}$ & \\
\hline \multirow[t]{2}{*}{18} & 133 & $4,9 \cdot 10^{-9}$ & $\mathrm{H}_{2} \mathrm{O}$ \\
\hline & 403 & $1,7 \cdot 10^{-9}$ & \\
\hline \multirow[t]{2}{*}{24} & 150 & $1,5 \cdot 10^{-12}$ & $\mathrm{C}_{2}$ \\
\hline & 388 & $8,5 \cdot 10^{-13}$ & \\
\hline \multirow[t]{2}{*}{26} & 147 & $1,5 \cdot 10^{-10}$ & $\mathrm{HC} \equiv \mathrm{CH}$ \\
\hline & 385 & $1,7 \cdot 10^{-11}$ & \\
\hline \multirow[t]{2}{*}{28} & 147 & $1,3 \cdot 10^{-9}$ & $\mathrm{CO}, \mathrm{H}_{2} \mathrm{C}=\mathrm{CH}_{2}$ \\
\hline & 401 & $5,0 \cdot 10^{-10}$ & \\
\hline \multirow[t]{2}{*}{29} & 145 & $7,0 \cdot 10^{-10}$ & $\mathrm{C}^{\cdot} \mathrm{H}=\mathrm{O}, \mathrm{H}_{3} \mathrm{C}\left(\mathrm{C}^{\cdot}\right) \mathrm{H}_{2}$ \\
\hline & 392 & $2,3 \cdot 10^{-10}$ & \\
\hline \multirow[t]{2}{*}{32} & 138 & $8,5 \cdot 10^{-11}$ & $\mathrm{CH}_{3} \mathrm{OH}, \mathrm{O}_{2}$ \\
\hline & 392 & $2,8 \cdot 10^{-10}$ & \\
\hline \multirow[t]{2}{*}{39} & 171 & $3,6 \cdot 10^{-9}$ & $\mathrm{H}_{2} \mathrm{C}=\mathrm{C}=\mathrm{C} \cdot \mathrm{H}, \mathrm{C} \cdot \mathrm{H}_{2} \mathrm{C} \equiv \mathrm{CH}$ \\
\hline & 384 & $3,6 \cdot 10^{-9}$ & \\
\hline \multirow[t]{2}{*}{41} & 144 & $6,0 \cdot 10^{-10}$ & $\mathrm{CH}_{3} \mathrm{CH}=\mathrm{CH}$ \\
\hline & 377 & $3,8 \cdot 10^{-10}$ & \\
\hline \multirow[t]{2}{*}{44} & 138 & $2,1 \cdot 10^{-10}$ & $\mathrm{CO}_{2}, \mathrm{CH}_{3} \mathrm{CHO}, \mathrm{CH}_{3} \mathrm{CH}_{2} \mathrm{CH}_{3}$ \\
\hline & 401 & $2,7 \cdot 10^{-10}$ & \\
\hline \multirow[t]{2}{*}{50} & 167 & $2,6 \cdot 10^{-12}$ & $\mathrm{HC} \equiv \mathrm{C}-\mathrm{C} \equiv \mathrm{CH}$ \\
\hline & 401 & $2,4 \cdot 10^{-12}$ & \\
\hline \multirow[t]{2}{*}{59} & 140 & $2,2 \cdot 10^{-11}$ & $\mathrm{COOCH}_{3}$ \\
\hline & 377 & $3,0 \cdot 10^{-11}$ & \\
\hline \multirow[t]{2}{*}{69} & 165 & $7,7 \cdot 10^{-11}$ & $\mathrm{H}_{2} \mathrm{C}=\mathrm{C}\left(\mathrm{CH}_{3}\right) \mathrm{CO}$ \\
\hline & 374 & $1,0 \cdot 10^{-10}$ & \\
\hline \multirow[t]{2}{*}{85} & 149 & $1,9 \cdot 10^{-11}$ & $\mathrm{H}_{2} \mathrm{C}=\mathrm{C}\left(\mathrm{CH}_{3}\right) \mathrm{COO}$ \\
\hline & 374 & $8,3 \cdot 10^{-12}$ & \\
\hline \multirow[t]{2}{*}{100} & 140 & $3,2 \cdot 10^{-12}$ & $\mathrm{H}_{2} \mathrm{C}=\mathrm{C}\left(\mathrm{CH}_{3}\right) \mathrm{COOCH}_{3}$ \\
\hline & 375 & $1,3 \cdot 10^{-11}$ & \\
\hline
\end{tabular}

Tabelle 5.1: Bei der Massenspektrometrie untersuchte Molmassen und deren mögliche Zusammensetzung nach Blanchet und Gupta [7, 41] sowie die Positionen der dabei auftretenden Intensitätspeaks. 


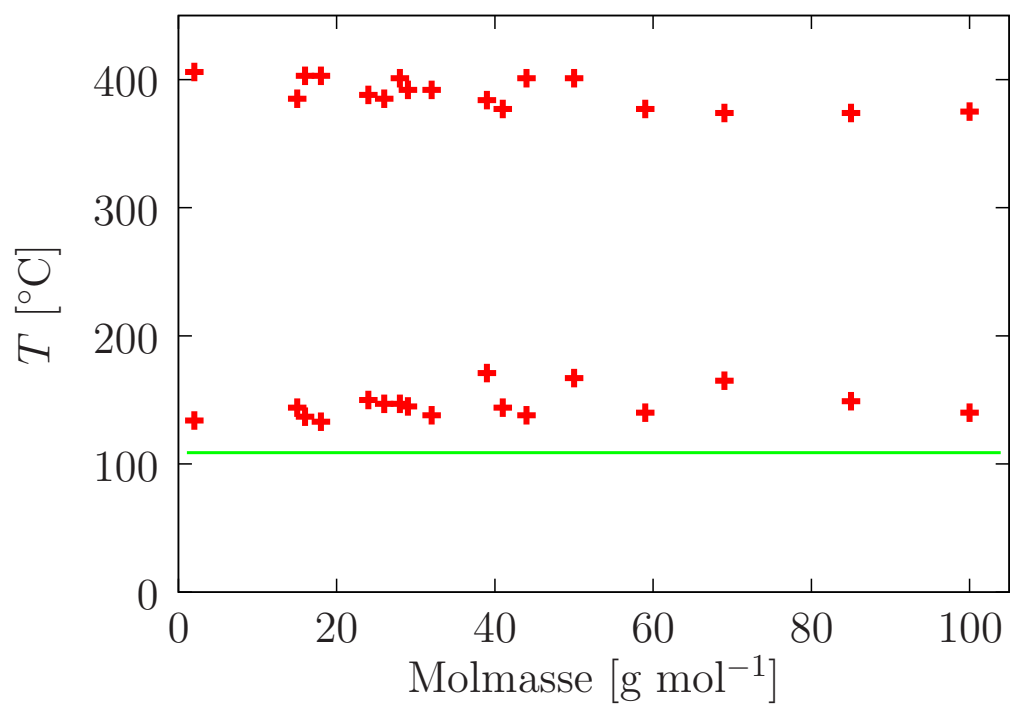

Abbildung 5.7: Auftragung der Temperaturen, für die Intensitätpeaks bei den Einzelmessungen der Massenspektrometrie auftreten, gegen alle detektierten Molmassen. Die Intensität selbst wird hierbei nicht berücksichtigt. Die Glasübergangstemperatur bei $T_{g}=109^{\circ} \mathrm{C}$ ist eingezeichnet.

bei nicht berücksichtigt, um die Verdeutlichung des hauptsächlichen Massenverlusts in zwei Stufen besser sichtbar zu machen. Die Moleküle von $\mathrm{H}_{2}$ bis zum Monomer lassen sich also entsprechend hauptsächlich bei Temperaturen im Intervall von 130 $150^{\circ} \mathrm{C}$ bzw. $370-410^{\circ} \mathrm{C}$ nachweisen. Bei dieser Auftragung wurde allerdings nicht berücksichtigt, welche Ionenstromintensität das jeweilige Peakmaximum überhaupt erreicht.

Im Folgenden soll das Abdampfverhalten anhand einiger wichtiger Moleküle diskutiert werden. Das Monomer wird z. B. nur wenig detektiert, was der geringe Ionenstrom zeigt (s. Abb. 5.6). Wie bei fast allen hier untersuchten Molekülmassen sind auch beim MMA hauptsächlich zwei Peaks zu erkennen. Diese Temperaturen entsprechen auch den beiden Stufen in Abbildung 5.3. Eine der in dieser Arbeit hauptsächlich detektierten Massen liegt bei $M_{w}=39 \mathrm{u}$ (Abb. 5.8 a), was hier als $\mathrm{H}_{2} \mathrm{C}=\mathrm{CCH}$ interpretiert wird. Betrachtet man das Monomer MMA (Abb. 5.8 d), entspricht $\mathrm{H}_{2} \mathrm{C}=\mathrm{CCH}$ dem Rest des Monomers ohne komplette Seitengruppe (Estergruppe) und mit abgespaltenem $\mathrm{H}_{2}$. Man erkennt hier zwar auch die zwei bereits erwähnten Peaks, allerdings liegt das Ionenstromsignal über den gesamten Temperaturbereich höher als bei fast allen anderen aufgenommenen Massenspektren des Schichtmaterials. Der komplette Monomeranteil ohne Seitengruppe wird verstärkt im ersten ausgezeichneten Temperaturbereich detektiert $\left(M_{w}=41 \mathrm{u}\right.$, Abb. $\left.5.8 \mathrm{~b}\right)$. Bei höheren Temperaturen löst sich stets Wasserstoff von der Methylgruppe, was wieder $M_{w}=39 \mathrm{u}$ entspricht. Der detektierte Wasserstoff $\mathrm{H}_{2}\left(M_{w}=2 \mathrm{u}\right.$, Abb. 5.8 c $)$ 

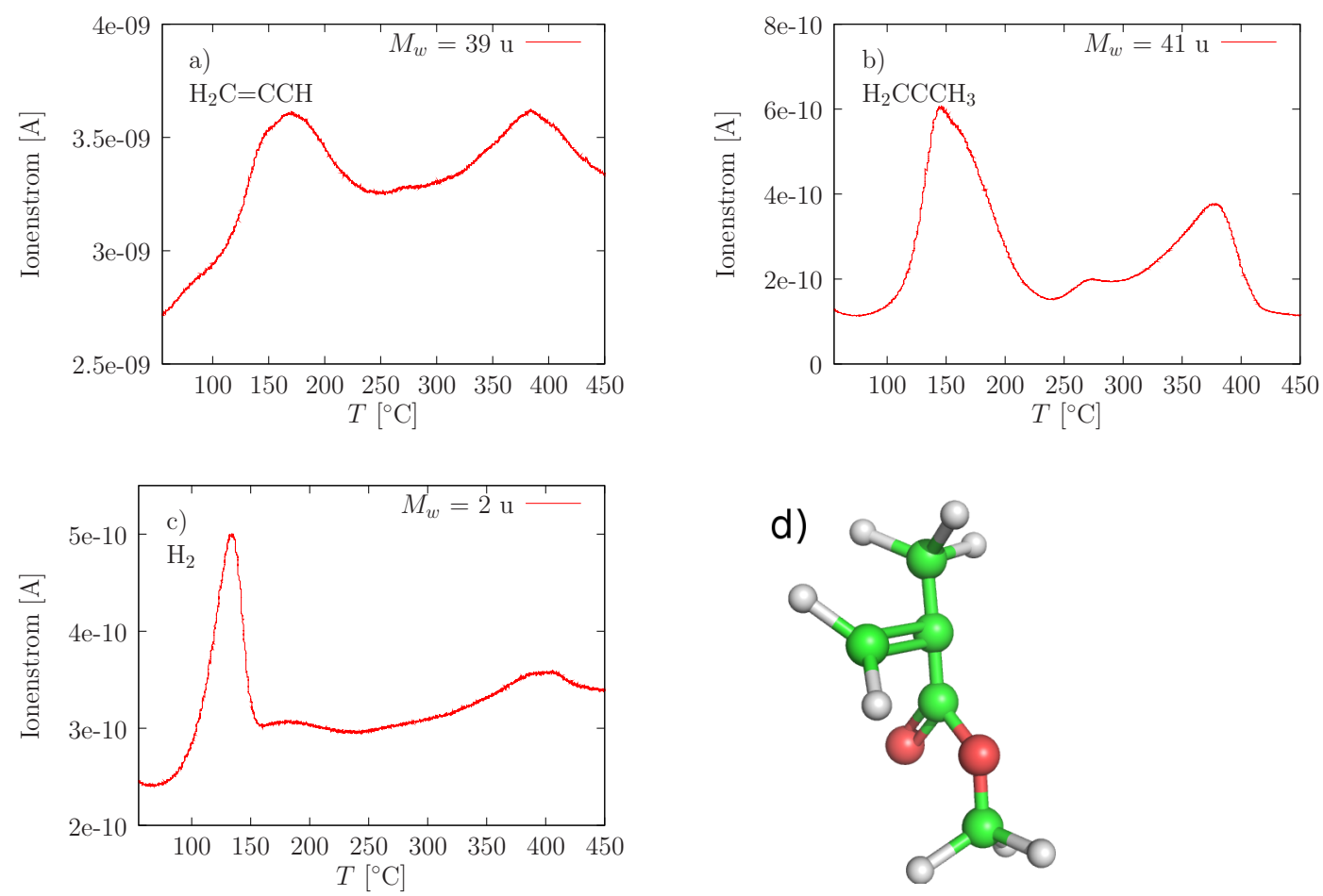

Abbildung 5.8: Massenspektrometrie-Messungen verschiedener Molekülmassen: a) $39 \mathrm{u}$ : $\mathrm{H}_{2} \mathrm{C}=\mathrm{CCH}$, b) $41 \mathrm{u}: \mathrm{H}_{2} \mathrm{C}=\mathrm{CCH}_{3}$, c) $2 \mathrm{u}: \mathrm{H}_{2}$; d) Visualisierung des Monomers MMA (C: grün, O: rot, $\mathrm{H}$ : weiß)

stammt hauptsächlich aus den beiden Methylgruppen des Ausgangsmaterials, wovon eine zur Seitengruppe gehörig ist. Der reine Wasserstoff wird allerdings bei höheren Temperaturen weniger detektiert, da er sich vermehrt mit anderen Atomen verbindet, was weiter unten noch diskutiert wird.

Während also der Hauptkettenanteil über den ganzen Temperaturbereich mit hohen Ionenstromintensitäten und für beide Massenverluststufen noch verstärkt detektiert werden konnte, unterscheiden sich die detektierten Schichtfragmente des Seitenkettenanteils klar von der ersten Stufe (etwa 130-150 ${ }^{\circ} \mathrm{C}$ ) zur zweiten Stufe (etwa $370-410^{\circ} \mathrm{C}$ ) in der Massenverlustmessung. Zunächst soll hier auf den unteren Temperaturbereich eingegangen werden.

Schon ab Beginn der Messung oberhalb der Raumtemperatur wird sehr viel Wasser $\left(\mathrm{H}_{2} \mathrm{O}\right)$ detektiert $\left(M_{w}=18 \mathrm{u}, \mathrm{Abb} .5 .9 \mathrm{a}\right)$. Dieses wird nicht zwingend aus der Probe frei, sondern dampft von der Oberfläche der Probe und des Messtiegels ab. Gleiches gilt in den folgenden Messungen für z. B. CO oder $\mathrm{CO}_{2}$, die sich stets als Adsorbate auf Oberflächen anlagern [72]. Unterhalb von $200^{\circ} \mathrm{C}$, wo der erste größere Materialverlust zu verzeichnen ist, setzt auch schon ein Abtrennen der kompletten Estergruppe ein $\left(M_{w}=59 \mathrm{u}\right.$, Abb. $\left.5.9 \mathrm{~b}\right)$, wobei aber auch schon eine weitere Spaltung der Seitengruppe zu bemerken ist. Die Carbonylgruppe (CO) selbst wird durch 

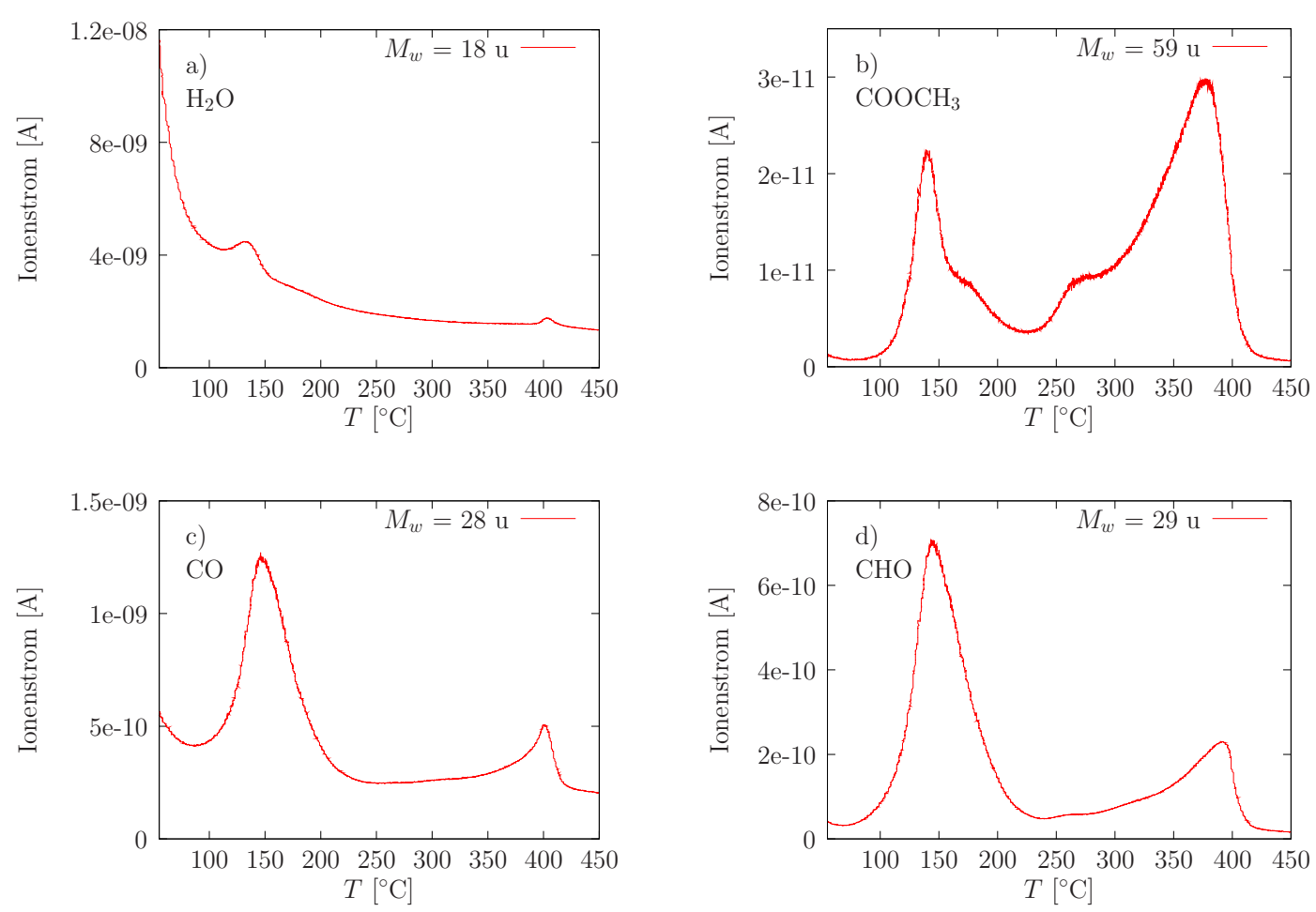

Abbildung 5.9: Massenspektrometrie-Messungen verschiedener Molekülmassen: a) $18 \mathrm{u}$ : $\mathrm{H}_{2} \mathrm{O}$, b) $59 \mathrm{u}: \mathrm{COOCH}_{3}$, c) $28 \mathrm{u}: \mathrm{CO}$, d) $29 \mathrm{u}: \mathrm{CHO}$

ihre Doppelbindung im Normalfall nicht gespalten, während die benachbarten Einzelbindungen leichter getrennt werden können. Aus diesem Grund wird diese Verbindung auch verstärkt gemessen $\left(M_{w}=28 \mathrm{u}\right.$, Abb. 5.9 $\left.\mathrm{c}\right)$, ebenso wie der Rest der Estergruppe ohne die Carbonylgruppe und mit abgespaltenem Wasserstoff (CHO: $\left.M_{w}=29 \mathrm{u}, \mathrm{Abb} .5 .9 \mathrm{~d}\right)$.

Im Vergleich zu den eben beschriebenen Fragmenten treten bei hohen Temperaturen oberhalb von $300{ }^{\circ} \mathrm{C}$ - entsprechend der zweiten Stufe in der Massenverlustmessung - kleinere Produkte häufiger auf. Es scheint in diesem Temperaturbereich ein Zerfall des Polymers in kleine stabile Moleküle vorzuliegen. So wird nun vermehrt das Molekül $\mathrm{CH}_{3}\left(M_{w}=15 \mathrm{u}, \mathrm{Abb} .5 .10 \mathrm{a}\right)$ detektiert. Ebenso wird für die Molmasse $M_{w}=16 \mathrm{u}$, was z. B. $\mathrm{CH}_{4}$ oder $\mathrm{O}$ entspricht (Abb. 5.10 b), mehr Material detektiert als bei niedrigeren Temperaturen. Sehr gut dazu passt auch der hohe gemessene Ionenstrom bei $M_{w}=32 \mathrm{u}$, also eine Detektierung von $\mathrm{CH}_{3} \mathrm{OH}$ bzw. $\mathrm{O}_{2}$ (Abb. 5.10 c). Außerdem ist hier nun mit $M_{w}=44 \mathrm{u}$ der Austritt von Kohlenstoffdioxid $\left(\mathrm{CO}_{2}\right.$, Abb. 5.10d) in gleichem Maße zu messen wie CO (vgl. Abb. 5.9c).

Die Massenspektren der Targetmessungen sind an dieser Stelle nicht gezeigt. Es ist aber dabei sehr gut zu erkennen gewesen, dass sich entsprechend dem starken Masseverlust ab $330^{\circ} \mathrm{C}$ (s. Abb. 5.3) hauptsächlich ein Peak in allen Spektren ausgebildet hat. Während das PMMA-Ausgangsmaterial bisher oberhalb der Glasüber- 

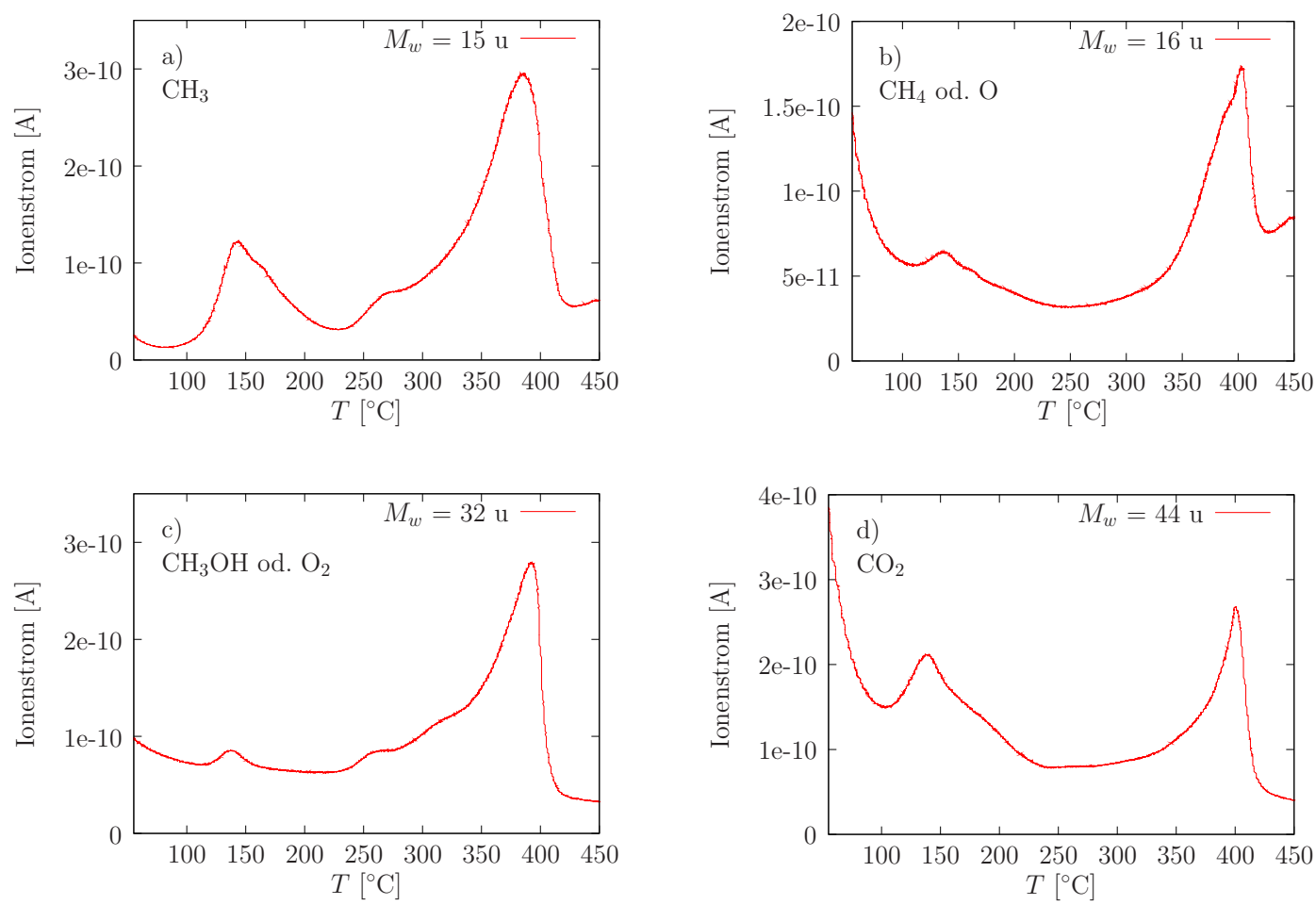

Abbildung 5.10: Massenspektrometrie-Messungen verschiedener Molekülmassen: a) $15 \mathrm{u}$ : $\mathrm{CH}_{3}$, b) 16 u: $\mathrm{CH}_{4}$ od. $\mathrm{O}$, c) $32 \mathrm{u}: \mathrm{CH}_{3} \mathrm{OH}$ od. $\mathrm{O}_{2}$, d) 44 u: $\mathrm{CO}_{2}$

gangstemperatur nur $10 \%$ seiner Masse verloren hat, dampft nun in einem sehr engen Temperaturfenster das gesamte verbliebene Material ab. Es bleibt hier also kein Rest zurück, wie wir ihn nach der thermischen Behandlung der PMMA-Schichten gefunden haben (s. Abb. 5.3). Zu bemerken ist dabei, dass fast alle ausgewählten Massen in gleicher Größenordnung der Intensität zu detektieren waren. So wurde hier das Monomer MMA (100 u) genauso stark abgedampft wie z. B. auch die einzelne Estergruppe $\left(59 \mathrm{u}\right.$ ). Nur die Verbindung $\mathrm{C}_{2}$ wurde hier mit zwei Größenordnungen kleinerer Intensität detektiert (vergleichbar mit dem Spektren der PMMA-Schicht), was daran liegt, dass diese Bindung allein instabil ist und sich deshalb bevorzugt mit anderen Atomen etwa $\mathrm{zu} \mathrm{CH}_{3}$ verbindet oder im Molekül $\mathrm{H}_{2} \mathrm{C}=\mathrm{CCH}$ verbleibt. Am stärksten detektiert wurde für das PMMA-Ausgangsmaterial neben $\mathrm{H}_{2} \mathrm{O}(18 \mathrm{u})$, welches auch schon bei $150^{\circ} \mathrm{C}$ einen starken Peak zeigt, die Methylgruppe $\mathrm{CH}_{3}(15 \mathrm{u})$ sowie der Monomeranteil ohne Seitengruppe $\mathrm{H}_{2} \mathrm{C}=\mathrm{CCH}(39 \mathrm{u})$ bzw. $\mathrm{H}_{2} \mathrm{CCCH}_{3}(41 \mathrm{u})$ und $\mathrm{H}_{2} \mathrm{C}=\mathrm{C}\left(\mathrm{CH}_{3}\right) \mathrm{CO}(69 \mathrm{u})$. Bei $150^{\circ} \mathrm{C}$ wurde für alle Massen jeweils ein sehr viel kleinerer Peak mitgemessen, der der ersten kleinen Stufe im Graph des Masseverlusts des Targetmaterials entspricht.

Während die hier gemessenen Massenspektren die Ablationsprodukte aus Pyrolyseuntersuchungen von BLANCHET und GUPTA wiederspiegeln, lässt sich mit den Ergebnissen von JELLINEK keine Übereinstimmung feststellen. Dort wurde hauptsäch- 
lich das Abdampfen des kompletten Monomers beschrieben [49].

Der in diesem Kapitel beschriebene, rein thermische Prozess erklärt nur einen Teil der ablaufenden Prozesse bei der Laserdeposition von PMMA. Aus diesem Grund zeigen Massenspektrometrie-Messungen während der Laserablation von LARCIPRETE andere Fragmentierungen des Materials [59]. Dort wurde für die Ablation des Materials ein Excimer-Laser mit $248 \mathrm{~nm}$ Wellenlänge verwendet und die Ablationsprodukte direkt unter Ionisierung mit einem ps-UV-Laser zur zeitaufgelösten Massenspektrometrie genutzt. Bei diesen Experimenten war auch eine Messung von Massen größer als die Monomermasse möglich. Die detektierten Massen passten nicht zur Fragmentierung der Polymerkette und damit nicht zu den hier gemessenen Fragmenten. Die Laserablation muss also zusätzlich zu den photothermisch ablaufenden Prozessen auch photochemische Prozesse beinhalten. KüPER beschreibt den Ablationsprozess sogar als rein photochemisch und diskutiert dies hauptsächlich über eine Seitenkettenabspaltung [56]. Aus SEC-Messungen wissen wir aber, dass durch den Depositionsprozess eine starke Hauptkettenspaltung stattfindet (s. Kap. 4.4). Dies zeigt widerum, dass die Laserdeposition von Polymeren nicht rein photochemisch ablaufen kann. Es muss also einen photothermischen und einen photochemischen Anteil geben.

Aufgrund ähnlicher Massenspektrometrie-Ergebnisse scheinen die stattfindenden Prozesse während der thermischen Behandlung von PMMA-Schichten vergleichbar mit den ablaufenden Prozessen während dem Beschuss von PMMA mit hochenergetischen Ionen in Bezug auf den Effekt des linearen Energietransfers (linear energy transfer, LET) 64]. Der LET gibt dabei an, wie viel Energie ein ionisierendes Teilchen pro Längeneinheit an ein durchdrungenes Material abgibt. Die Ergebnisse bestehen aus Spaltungsprodukten des Polymers und deren Reaktionsprodukten sowie Spaltfragmente, verursacht durch die dort verwendete Messmethode (residual gas analyzer, RGA). So ist dort wie in dieser Arbeit z. B. die Massenzahl 59, welche als $\mathrm{CH}_{3} \mathrm{OOC} \bullet$ angenommen wird, nur mit vergleichsweise geringer Intensität zu detektieren. Da ein hoher Austritt von kleinen sauerstoffhaltigen Molekülen verzeichnet werden konnte, wird daraus ersichtlich, dass ein großer Anteil der Methylestergruppen aufgespalten wurde. Größere Moleküle scheinen es allgemein schwer zu haben, aus dem Material zu diffundieren. Dadurch muss ein großer Anteil erst durch zusätzliche Bestrahlung dissoziieren, bevor die Moleküle das Substrat verlassen können.

Es ergibt sich so, dass anhängende Atome $\left(\mathrm{H}^{\bullet}\right)$ und Seitengruppen $\left(\mathrm{CH}_{3}{ }^{\bullet}, \mathrm{CH}_{3} \mathrm{O}^{\bullet}\right.$, $\mathrm{CH}_{3} \mathrm{OOC}^{\bullet}$ ) allgemein zur Spaltung neigen. Der Austritt zahlreicher, ungesättigter Kohlenwasserstoffe wie ${ }^{\bullet} \mathrm{CH}=\mathrm{C}=\mathrm{CH}_{2}(39 \mathrm{u})$ oder $\mathrm{CH}_{3} \mathrm{CH}=\mathrm{C} \bullet \mathrm{H}(41 \mathrm{u})$ deutet außerdem eine erhebliche Spaltung der Polymerhauptkette (backbone) an. Würde die Hauptkette nur wenig gespalten werden, wären weniger Kettenenden und damit weniger ungesättigte Kohlenwasserstoffe zu verzeichnen. Diese können als Ketten mit $\mathrm{C}_{2^{-}}, \mathrm{C}_{3^{-}}$und $\mathrm{C}_{4}$-Kohlenwasserstoffen auftreten, die durch $\mathrm{H}^{\bullet}-, \mathrm{CH}_{3}$ und $\mathrm{CH}_{3} \mathrm{OOC} \bullet$-Radikale verarmt sind, so z. B. $\mathrm{HC} \equiv \mathrm{CH}, \mathrm{CH}_{2}=\mathrm{C}=\mathrm{CH}_{2},{ }^{\bullet} \mathrm{CH}=\mathrm{C}=\mathrm{CH}_{2}$, $\mathrm{CH} \equiv \mathrm{C}-\mathrm{C} \equiv \mathrm{CH}, \mathrm{CH} \equiv \mathrm{C}-\mathrm{C} \equiv \mathrm{C}^{\bullet}$. Berechnungen zur Fragmentierung bei Elektronen- 
bestrahlung von normalen Alkanen ergaben, dass es sehr viel wahrscheinlicher ist $\mathrm{C}_{3^{-}}$und $\mathrm{C}_{4}$-Verbindungen zu brechen als $\mathrm{C}_{1}$ - und $\mathrm{C}_{2}$-Verbindungen [108].

So können nach LEE - unter Verwendung der Messdaten von TODD [105] und VenkATESAN [107] - die verschiedenen Fragmente, die durch Kettenspaltung und anschließende chemische Reaktionen aus dem PMMA entstanden sind, hauptsächlich in drei Gruppen unterteilt werden [64]. P steht hierbei immer für die PMMAHauptkette:

- In Gruppe I lassen sich die dominanten Verbindungen aus den Wasserstoff$\left(\mathrm{H}^{\bullet}\right)$ und Methylradikalen $\left(\mathrm{CH}_{3}^{\bullet}\right)$ ableiten:

$$
\begin{aligned}
\mathrm{P}-\mathrm{H} & \longrightarrow \mathrm{P}^{\bullet}+{ }^{\bullet} \mathrm{H} \\
\mathrm{P}+\mathrm{H}^{\bullet} & \longrightarrow \mathrm{P}^{\bullet}+\mathrm{H}_{2} \\
\mathrm{H}^{\bullet}+{ }^{\bullet} \mathrm{H} & \longrightarrow \mathrm{H}_{2} \\
\mathrm{P}-\mathrm{CH}_{3} & \longrightarrow \mathrm{P}^{\bullet}+{ }^{\bullet} \mathrm{CH}_{3} \\
\mathrm{P}+\mathrm{H}_{3} \mathrm{C}^{\bullet} & \longrightarrow \mathrm{P}^{\bullet}+\mathrm{CH}_{4} \\
\mathrm{H}_{3} \mathrm{C}^{\bullet}+{ }^{\bullet} \mathrm{H} & \longrightarrow \mathrm{CH}_{4} \\
\mathrm{H}_{3} \mathrm{C}^{\bullet}+{ }^{\bullet} \mathrm{CH}_{3} & \longrightarrow \mathrm{H}_{3} \mathrm{C}-\mathrm{CH}_{3}
\end{aligned}
$$

- Die Gruppe II wird bestimmt durch Methoxy- $\left(\bullet \mathrm{OCH}_{3}\right)$ und Methylesterradikale $\left(\mathrm{CH}_{3} \mathrm{OOC}\right)^{\bullet}$ :

$$
\begin{aligned}
& \mathrm{P}-\mathrm{COOCH}_{3} \longrightarrow \mathrm{P}^{\bullet}+{ }^{\bullet} \mathrm{COOCH}_{3} \\
& \mathrm{P}-\mathrm{COOCH}_{3} \longrightarrow \mathrm{P}-\mathrm{C}(\mathrm{O})^{\bullet}+{ }^{\bullet} \mathrm{OCH}_{3} \\
& { }^{\bullet} \mathrm{COOCH}_{3} \longrightarrow{ }^{\bullet} \mathrm{CH}_{3}+\mathrm{CO}_{2} \\
& { }^{\bullet} \mathrm{OCH}_{3} \longrightarrow \mathrm{HCHO}+{ }^{\bullet} \mathrm{H} \\
& \mathrm{P}+\mathrm{CH}_{3} \mathrm{O}^{\bullet} \longrightarrow \mathrm{P}^{\bullet}+\mathrm{CH}_{3} \mathrm{OH} \\
& \mathrm{CH}_{3} \mathrm{O}^{\bullet}+{ }^{\bullet} \mathrm{H} \longrightarrow \mathrm{CH}_{3} \mathrm{OH} \\
& \mathrm{CH}_{3} \mathrm{O}^{\bullet}+{ }^{\bullet} \mathrm{CH} \longrightarrow \mathrm{CH}_{3} \mathrm{OCH}
\end{aligned}
$$

- In Gruppe III werden ungesättigte Verbindungen wie die sauerstofffreien Alkane und die möglicherweise zyklischen Kohlenwasserstoffe zusammengefasst, die von der Hauptkette des Polymers stammen:

$\mathrm{P}$ (Verlust von anhängenden Gruppen und Gruppen wie $\bullet \mathrm{H}$ und ${ }^{\bullet} \mathrm{COOCH}_{3}$ )

$\longrightarrow$ verarmte $\mathrm{P}^{\prime}$

$\longrightarrow$ Abspaltung von Kettenendsegmenten

$\longrightarrow$ verarmte Kohlenwasserstoffe 


\subsection{Fazit}

Bei den thermischen Untersuchungen von laserdeponierten PMMA-Schichten konnte festgestellt werden, dass die Glasübergangstemperatur $T_{g}$ zwar gegenüber dem Ausgangsmaterial abgesenkt war, die Absenkung aber bei weitem nicht in dem Maße statt fand, wie es für die starke Kettenlängenreduktion in den Schichten erwartet wurde. Dieses hohe $T_{g}$ wird unter anderem darauf zurückgeführt, dass das laserdeponierte PMMA eine Verarmung an Seitenketten aufweist. Dies wirkt umgekehrt auf die Glasübergangstemperatur wie die bekannte Abnahme der Glasübergangstemperatur für längere Seitenketten bei höheren Poly(alkyl methacrylat)en (PAMA). Die abgespaltenen Estergruppen oder Fragmente, die schon in der Schicht vorlagen, konnten bei Temperaturen oberhalb der Glasübergangstemperatur durch Massenspektrometrie nachgewiesen werden, da bei diesen Temperaturen die Mobilität ausreicht, um die Moleküle aus der Schicht abdampfen zu lassen. Außerdem finden bei diesen Temperaturen auch schon Spaltungen statt, durch die Quervernetzungen ermöglicht werden. So macht sich bis $200^{\circ} \mathrm{C}$ ein Masseverlust von $40 \%$ bemerkbar, aber gleichzeitig ist ein Anstieg der mittleren Molmasse zu verzeichnen. Durch diesen Anstieg der mittleren Molmasse mit der Temperatur wird ersichtlich, dass bei dem Vergleich mit der Literatur eine zu geringe Kettenlänge angenommen wurde. Damit verschiebt sich unser Messwert auch wieder näher an die Literaturkurve der Glasübergangstemperaturen für die verschiedenen Kettenlängen von PMMA.

Durch die massenspektrometrische Analyse der Schichtfragmente konnte der zweistufige Masseverlust als bimodale Verteilung in den Massenspektren verifiziert werden. Die Fragmente, die hauptsächlich zwischen $130-150{ }^{\circ} \mathrm{C}$ detektiert wurden, sprechen hauptsächlich für eine Aufspaltung des Monomers mit Wasserstoffabspaltung und einer Teilung der Estergruppe in Carbonylgruppen (CO) und Aldehyde (CHO). Für die Abspaltung von Estergruppen spricht ebenfalls ein Verlust in der Intensität der gemessenen $\pi_{\mathrm{C}=\mathrm{O}}^{*}$-Bindung. Der entsprechende Anstieg in der Intensität der $\pi_{\mathrm{C}=\mathrm{C}^{-}}^{*}$ Bindung zeigt eine Umlagerung der frei gewordenen Bindungen nicht nur zu benachbarten Ketten (Quervernetzung) sondern auch zur eigenen Kette (Doppelbindungsbildung).

Die zweite Stufe im Masseverlust der PMMA-Schicht zwischen $370-410^{\circ} \mathrm{C}$ bzw. der hauptsächliche Masseverlust des PMMA-Targets oberhalb von $330^{\circ} \mathrm{C}$ zeigen die thermische Zersetzung des Materials. Für das deponierte Schichtmaterial lassen sich nun vermehrt viel kleinere Fragmente detektieren, die mittlere Molmasse verringert sich wieder.

Dass diese thermische Modifikation des PMMA auch Einfluss auf die Morphologie und vor allem auf die Härte der deponierten Schichten hat, wird in Kapitel 6.3 gezeigt werden. Im Folgenden sollen auch andere Möglichkeiten diskutiert werden, wie die Oberflächenmorphologie sowie die chemischen und mechanischen Eigenschaften wie die Härte von glatten PMMA-Schichten beeinflusst werden können. Untersucht wurden dabei vor allem die aus der Lithographie bekannte Methode der Härtung durch 
Bestrahlung mit ultraviolettem Licht (Kap. 6.1) und die Härtung durch Einbringung einer vernetzenden Komponente in das Targetmaterial, mit dem dann Schichten laserdeponiert wurden (Kap. 6.2). Die verschiedenen Methoden werden dann miteinander verglichen. 


\section{Modifizierung glatter PMMA-Schichten}

In diesem Kapitel soll nun diskutiert werden, wie die Eigenschaften der glatten PMMA-Schichten (s. Kap. 4) beeinflusst werden können. Während die Spaltung von PMMA-Ketten direkt anhand von geringeren Molmassen aus SEC-Messungen gezeigt werden kann, kann auf den Vernetzungsgrad des Polymers dagegen über die Härteänderungen des Materials geschlossen werden [62]. Deshalb sollen neben der Oberflächenmorphologie jeweils die chemischen Eigenschaften und vor allem die mechanischen Eigenschaften untersucht werden. Mit dem Ziel eine Härtung der Schichten zu erreichen, soll zunächst auf die aus der Anwendung von PMMA als Photolack in der Lithographie bekannte Härtung durch Einstrahlung von ultraviolettem Licht eingegangen werden (Kap. 6.1). Danach wird diskutiert, welchen Einfluss das Einbringen eines vernetzenden Materials wie Bisphenol A Dimethacrylat in das PMMATarget auf die daraus deponierten Schichten hat (Kap. 6.2). Und schließlich wird noch in Bezug auf Kapitel 5 die Morphologie- und Härteänderung diskutiert, wie sie aus thermischer Behandlung resultiert (Kap. 6.3).

\subsection{Modifizierung durch UV-Bestrahlung der Schichten}

Dass sich PMMA durch hohen Energieeintrag vernetzen bzw. härten lässt, ist z. B. aus der Anwendung dieses Polymers als negativer Fotolack in der ElektronenstrahlLithographie bereits bekannt [111]. Verwendet man dagegen geringe Intensitäten bei der Belichtung, wird der PMMA-Photolack lediglich zerstört, ohne zu vernetzen. Auf diese Weise kann man PMMA als positiven Photolack verwenden [47. DAVEnAs beschrieb die Wandlung von PMMA von einem positiven Photolack (löslich) in einen negativen Photolack (nicht löslich) durch einen hohen Fluenzeintrag [16]. Anstatt eines Elektronenstrahls kann zur Belichtung des PMMA-Photolacks auch ultraviolette Strahlung verwendet werden [38]. Inwiefern sich eine Vernetzung bzw. Härtung von PMMA durch UV-Strahlung auf laserdeponierte, glatte PMMA-Schichten übertragen lässt und in welchem Ausmaß eine Änderung der Schichteigenschaften stattfindet, soll hier diskutiert werden. 


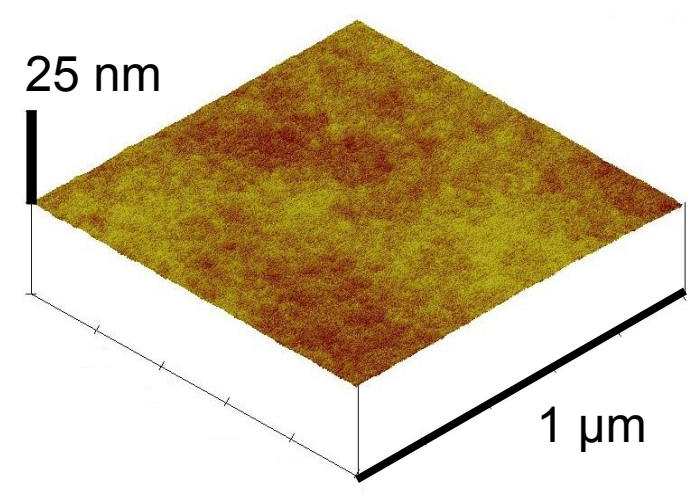

Abbildung 6.1: AFM-Aufnahme einer PMMA-Schicht hergestellt mit 1.000 Pulsen bei $113 \mathrm{~mJ} \mathrm{~cm}^{-2}$ nach 1 Stunde UV-Bestrahlung und einem Abstand zur Lampe von $3 \mathrm{~cm}$. Die RMS-Rauigkeit ändert sich gegenüber der unbestrahlten Schicht nicht und liegt somit immer noch bei $\sigma_{\mathrm{RMS}}=0,3 \mathrm{~nm}$.

\subsubsection{Morphologie}

Durch die UV-Bestrahlung der PMMA-Schichten werden chemische Bindungen des Polymers stark beeinflusst, eine makroskopische Änderung der Morphologie ist dabei aber nicht festzustellen (Abb. 6.1). So liegt die RMS-Rauigkeit, die aus der AFMAufnahme einer $1 \mathrm{~h}$ bestrahlten Schicht in Abbildung 6.1 ermittelt wurde, bei $\sigma_{\mathrm{RMS}}=$ 0,3 nm. Dies entspricht den Rauigkeiten, die auch für die unbestrahlten PMMASchichten beobachtet wurden (vgl. Abb. 3.7).

Es wurde mit Hilfe eines Thermoelements an der Probenposition unter der UVLampe nachgewiesen, dass die hier verwendete UV-Strahlung (s. Kap. 2.1.3) an den Proben eine Temperaturerhöhung bis maximal $80^{\circ} \mathrm{C}$ bewirkt. Nach den Untersuchungen zum thermischen Einfluss auf das laserdeponierte PMMA aus Kapitel 5 und den entsprechenden Härtemessungen bei dieser Temperatur (s. Kap. 6.3) kann davon ausgegangen werden, dass die hier beobachteten Veränderungen vor allem in Bezug auf die Härtung des Material auf die Behandlung mit UV-Strahlung zurückzuführen sind.

Anders stellt sich diese Erkenntnis für Experimente an laserdeponierten PEMASchichten dar. Da bei PEMA das Ausgangsmaterial lediglich eine Glasübergangstemperatur von $T_{g}=65^{\circ} \mathrm{C}$ aufweist, wurde im Rahmen der Bachelorarbeit von EBerL ein Abstand des Lichtleiters zur Probe von $10 \mathrm{~cm}$ gewählt [24]. Hier betrug die Temperatur an der Probe dann etwa $30^{\circ} \mathrm{C}$. Die UV-Strahlung verteilte sich dadurch aber auch auf eine größere Fläche, weshalb die Proben dort etwas länger bestrahlt wurden, um eine vergleichbare Modifikation zu erlangen. Außerdem wurde dort das Verhalten der Schichten bei Langzeitbestrahlung untersucht. Die Ergebnisse zeigten einen deutlichen Unterschied zwischen PMMA und PEMA. Die PMMA- 


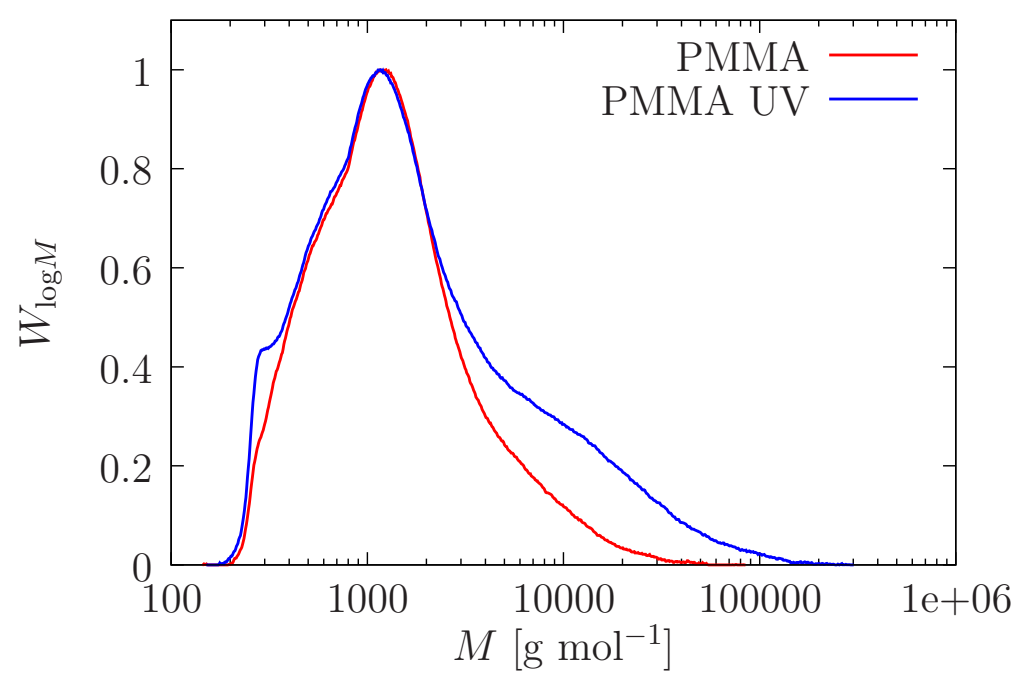

Abbildung 6.2: SEC-Messungen von PMMA-Schichten hergestellt bei $130 \mathrm{~mJ} \mathrm{~cm}{ }^{-2}$ nach UV-Bestrahlung und zum Vergleich unbehandelt. Die mittlere Molmasse von unbehandeltem PMMA ergab $\bar{M}_{w}=2.200 \mathrm{~g} \mathrm{~mol}^{-1}$ mit einer Polydispersität von $D=2,3$. Nach einer Bestrahlungszeit von $1 \mathrm{~h}$ in einem Abstand zur Lampe von $3 \mathrm{~cm}$ erhöht sich die mittlere Molmasse auf $\bar{M}_{w}=5.100 \mathrm{~g} \mathrm{~mol}^{-1}$ mit $D=5,0$.

Schichten verloren während einer Bestrahlung von über 2 Wochen Schichtmaterial in dem Maße, dass das Substrat teilweise blank zu sehen war. Die verbliebene PMMASchicht blieb glatt und geschlossen. Bei PEMA dagegen zeigte die zuvor geschlossene Schicht nach der Bestrahlung und dem dadurch resultierten Schichtverlust wieder starke Entnetzung vom Substrat, wie es schon für weniger dicke PEMA-Schichten direkt nach der Herstellung zu bemerken war (s. Kap. 4.2).

\subsubsection{Chemische Eigenschaften}

Nachdem die PMMA-Schichten nach der UV-Bestrahlung keine makroskopischen Veränderungen an der Oberfläche gezeigt haben, soll nun untersucht werden, ob das UV-Licht mikroskopisch Einfluss auf die chemischen Bindungen im Material hat.

Die Molmassenverteilung der UV-bestrahlten PMMA-Schicht zeigt die Ausbildung einer Schulter zu kleineren Molmassen hin (Abb. 6.2). Diese SEC-Messungen weisen klar auf eine Spaltung der Ketten durch die zusätzliche Bestrahlung hin. Da sich freie Radikale aber neue Bindungpartner suchen, führt diese Spaltung außerdem zu einer Quervernetzung des Materials. Dadurch ist zu höheren Molmassen hin eine entsprechend stärker ausgeprägte Schulter zu erkennen. Damit erhält man insgesamt ein Anwachsen der mittleren Molmassen von $\bar{M}_{w}=2.200 \mathrm{~g} \mathrm{~mol}^{-1}$ für die unbestrahlte PMMA-Schicht auf $\bar{M}_{w}=5.100 \mathrm{~g} \mathrm{~mol}^{-1}$ nach $1 \mathrm{~h} \mathrm{UV-Bestrahlung}$. Die Polydispersität steigt entsprechend von $D=2,3$ auf $D=5,0$. 


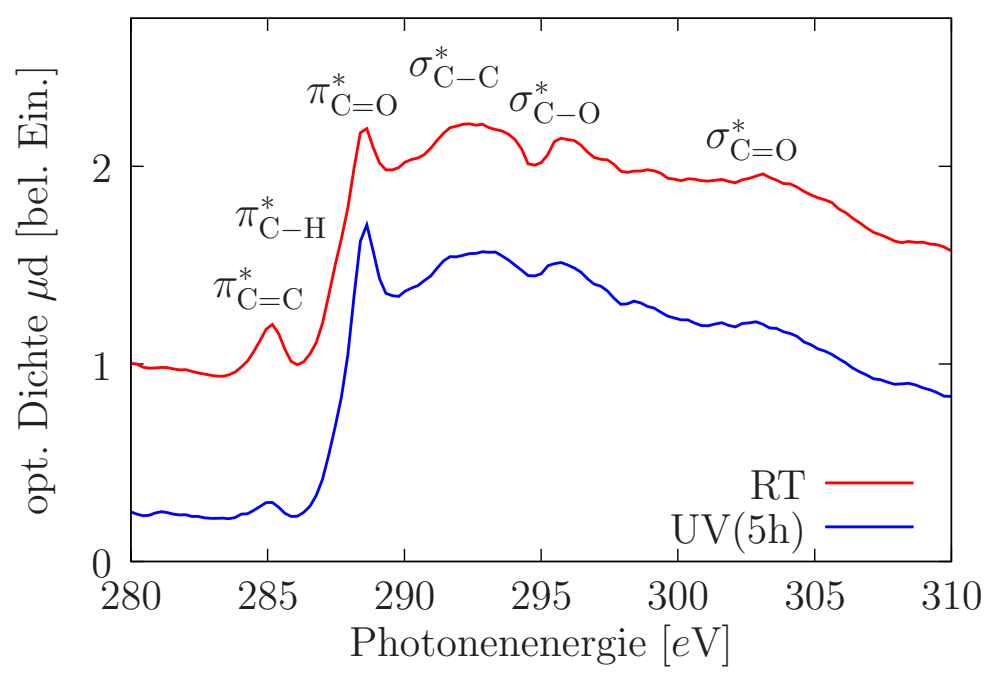

Abbildung 6.3: NEXAFS-Messungen einer bei Raumtemperatur mit 1.100 Pulsen bei $125 \mathrm{~mJ} \mathrm{~cm}^{-2}$ hergestellten etwa $250 \mathrm{~nm}$ dicken PMMA-Schicht und einer entsprechend bei Raumtemperatur hergestellten und nachträglich für $5 \mathrm{~h}$ UV-bestrahlten Schicht.

Vergleichbar zu diesen Ergebnissen gibt LEE einen guten Überblick über die Vernetzungs- und Spaltungsmechanismen von PMMA durch Bestrahlung mit hochenergetischen Ionen in Bezug auf den Effekt des linearen Energietransfers (linear energy transfer, LET) [64]. Messungen zeigen dabei ebenfalls das Resultat von kürzeren und längeren Ketten, was auf das simultane Auftreten von Spaltung und Vernetzung zurückgeführt wird.

Welche Bindungen bei diesen Prozessen beteiligt sind, lässt sich unter anderem anhand von NEXAFS-Messungen diskutieren (Abb. 6.3). Verglichen wurde hier eine unbestrahlte Schicht (s. Kap. 4.4 mit einer Schicht, die nach der Herstellung für $5 \mathrm{~h}$ in einem Abstand von $10 \mathrm{~cm}$ zum Lichtleiter UV-bestrahlt wurde. Vor allem ist bei diesem Vergleich durch die UV-Strahlung eine Verkleinerung des Peaks bei $285 \mathrm{eV}$ $\mathrm{zu}$ bemerken, was der $\pi_{\mathrm{C}=\mathrm{C}^{-}}^{*}$ Bindung entspricht. Warum dagegen die $\pi_{\mathrm{C}=\mathrm{O}^{-}}^{*}$ Bindung bei $288 \mathrm{eV}$ etwas an Intensität zugenommen hat, kann hier nicht erklärt werden. Die $\mathrm{C}=\mathrm{C}$-Doppelbindungen, die durch die Inkubation beim Depositionsprozess im Material entstanden sind, wurden durch die nachträgliche UV-Bestrahlung wieder aufgebrochen. Aufspaltung von Bindungen kommt hier zustande, da bei der Bestrahlung des Polymers angeregte Atome und Ionen erzeugt werden und dann durch Coulomb-Wechselwirkung zwischen diesen Ionen eine zu starke Bindungsdehnung oder ein Bindungsbruch verursacht werden kann [64]. Die dadurch entstandenen freien Bindungen können sich dann wieder neue Bindungspartner suchen und so z. B. quervernetzen. Dies sollte Auswirkungen auf die Härte der Schichten haben, was nun diskutiert werden soll. 


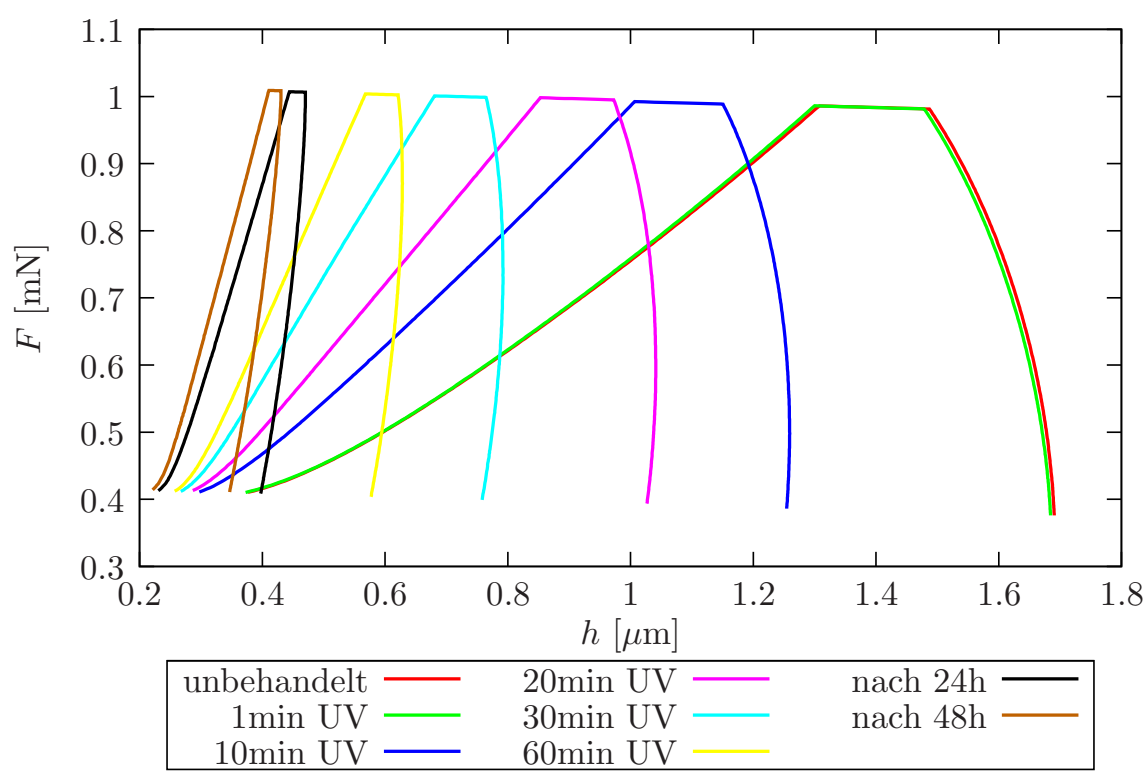

Abbildung 6.4: Aus den Kraft-Eindringtiefe-Kurven lässt sich für zunehmende UV-Bestrahlungszeiten eine Abnahme der Plastizität aus der Rückkurve und eine Zunahme der Härte aus der geringeren Eindringtiefe bei der Maximalkraft von $1 \mathrm{mN}$ ableiten. Misst man die 60 min bestrahlte Probe nach einem bzw. zwei Tagen nochmal, lässt sich eine starke Abhängigkeit von der Wartezeit zwischen UV-Bestrahlung und Härtemessung feststellen.

\subsubsection{Mechanische Eigenschaften}

Um gezielte Aussagen über den Einfluss der UV-Strahlung auf die Härte der laserdeponierten PMMA-Schichten machen zu können, wurden für eine Schicht mehrmals die Kraft-Eindringtiefe-Kurven bis zu einer maximalen Bestrahlzeit von $1 \mathrm{~h}$ aufgenommen (Abb. 6.4). Mit zunehmender Bestrahldauer lässt sich aus den Rückkurven dieser Messungen eine Abnahme der Plastizität und aus den geringeren Eindringtiefen bei der Maximalkraft von $1 \mathrm{mN}$ eine Zunahme der Härte ableiten. Nach Beendigung der Bestrahlung wurde die Schicht nach $24 \mathrm{~h}$ bzw. nach $48 \mathrm{~h}$ nochmals vermessen. Es ist zu bemerken, dass die Kurven nicht deckungsgleich sind, sondern dass die bestrahlten Schichten anscheinend mit der Zeit weiterhin verhärten.

Betrachtet man direkt die sich ergebenden Härtewerte, ist gut zu erkennen, dass die Universalhärte mit der Bestrahlungszeit linear zunimmt, sofern die Messungen immer zeitnah nach der jeweiligen Bestrahlung durchgeführt wurden (Abb. 6.5). Wartet man einige Zeit zwischen dem Ende der jeweiligen Bestrahlung und der Härtemessung führt dies sofort zu einer Abweichung des linearen Härteanstiegs. Diese Wartezeiten, die nach der letzten Bestrahlung bis zu 2 Tage ausgedehnt wurden, sind in Abbildung 6.5 mit Pfeilen gekennzeichnet. Besonders zu erwähnen sind hier die Messwerte nach 40 min und 50 min Betrahlungszeit. Obwohl die Schicht nach 40 min 


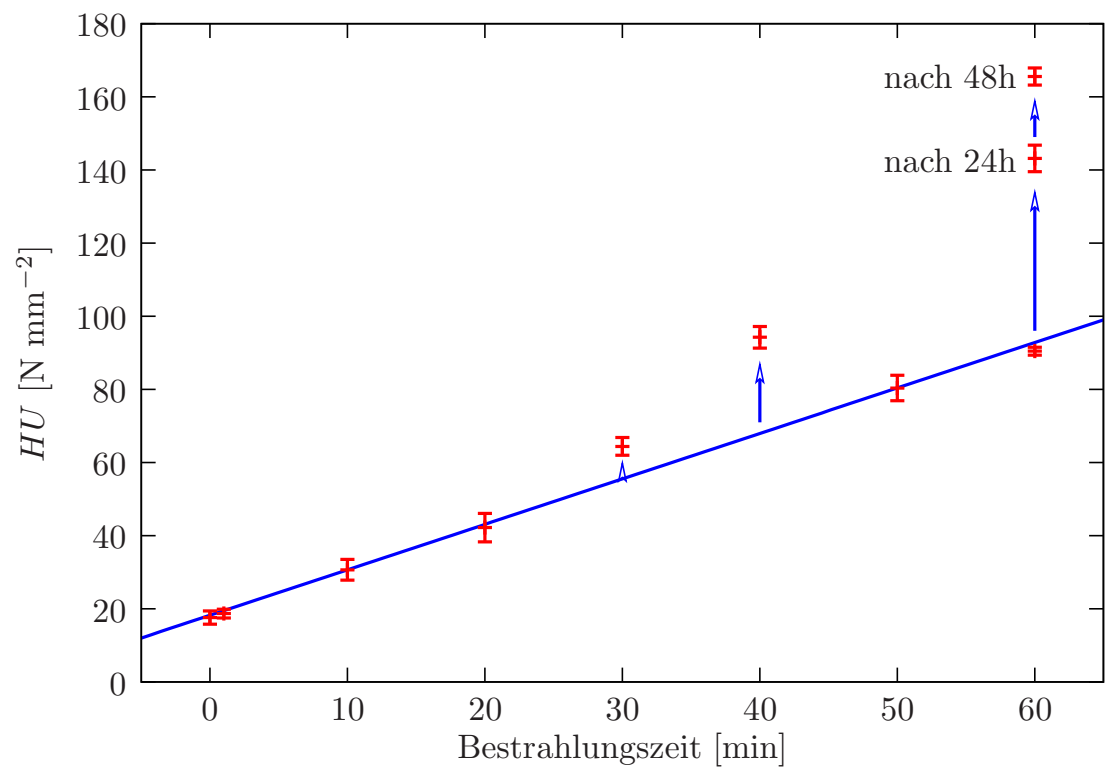

Abbildung 6.5: Härteanstieg mit zunehmender Bestrahlungszeit. Die Messwerte sind stark abhängig von der Wartezeit zwischen UV-Bestrahlung und Härtemessung. Längere Wartezeiten sind hier durch Pfeile gekennzeichnet.

Bestrahlung durch die etwas längere Wartezeit vor der Härtemessung bereits eine Universalhärte von $H U=94 \pm 3 \mathrm{~N} \mathrm{~mm}^{-2}$ auswies, ist der Härtewert nach erneuter Bestrahlung wieder auf $H U=80 \pm 3 \mathrm{~N} \mathrm{~mm}^{-2}$ gesunken und führt so den linearen Härteanstieg wieder fort. Durch die zusätzliche Bestrahlung werden also neue Radikale gebildet, die direkt nach der Bestrahlung noch keine Bindungspartner gefunden haben, dadurch ist die Schicht kurzfristig wieder weicher.

Dieses Langzeitverhalten wurde noch genauer untersucht, indem sowohl zwei verschieden gelagerte PMMA-Schichten als auch zwei bestrahlte Schichten $-1 \mathrm{~h}$ bzw. $24 \mathrm{~h}$ Bestrahlungszeit - über mehrere Monate hinweg vermessen wurden. Vergleicht man jeweils den ersten mit dem letzten Messwert, kann man feststellen, dass die unbestrahlten Proben eine Härtezunahme von $H U=4,6 \pm 0,7 \mathrm{~N} \mathrm{~mm}^{-2}$ auf $H U=$ $13 \pm 3 \mathrm{~N} \mathrm{~mm}^{-2}$ für eine dunkel gelagerte Schicht bzw. für die im Exsikkator mit Trockenperlen gelagerte Schicht eine Härtezunahme von $H U=4,2 \pm 0,4 \mathrm{~N} \mathrm{~mm}^{-2}$ auf $H U=22 \pm 1 \mathrm{~N} \mathrm{~mm}^{-2}$ aufweisen. Die für $1 \mathrm{~h}$ bestrahlte Schicht hat ihre Härte von $H U=75,2 \pm 0,7 \mathrm{~N} \mathrm{~mm}^{-2}$ nach 8 Monaten $\mathrm{zu} H U=143 \pm 2 \mathrm{~N} \mathrm{~mm}^{-2}$ geändert und die für $24 \mathrm{~h}$ bestrahlte Schicht entsprechend von $H U=178 \pm 7 \mathrm{~N} \mathrm{~mm}^{-2} \mathrm{zu}$ $H U=239 \pm 13 \mathrm{~N} \mathrm{~mm}^{-2}$. Die unbestrahlten Schichten zeigen sich bei dieser Langzeitmessung für mindestens 3 Wochen sehr stabil in Bezug auf ihre Härte. In der logarithmischen Auftragung zeigen dagegen die UV-bestrahlten Schichten einen linearen Anstieg. Dies bedeutet, dass vor allem in den ersten Tagen nach der Bestrahlung die 


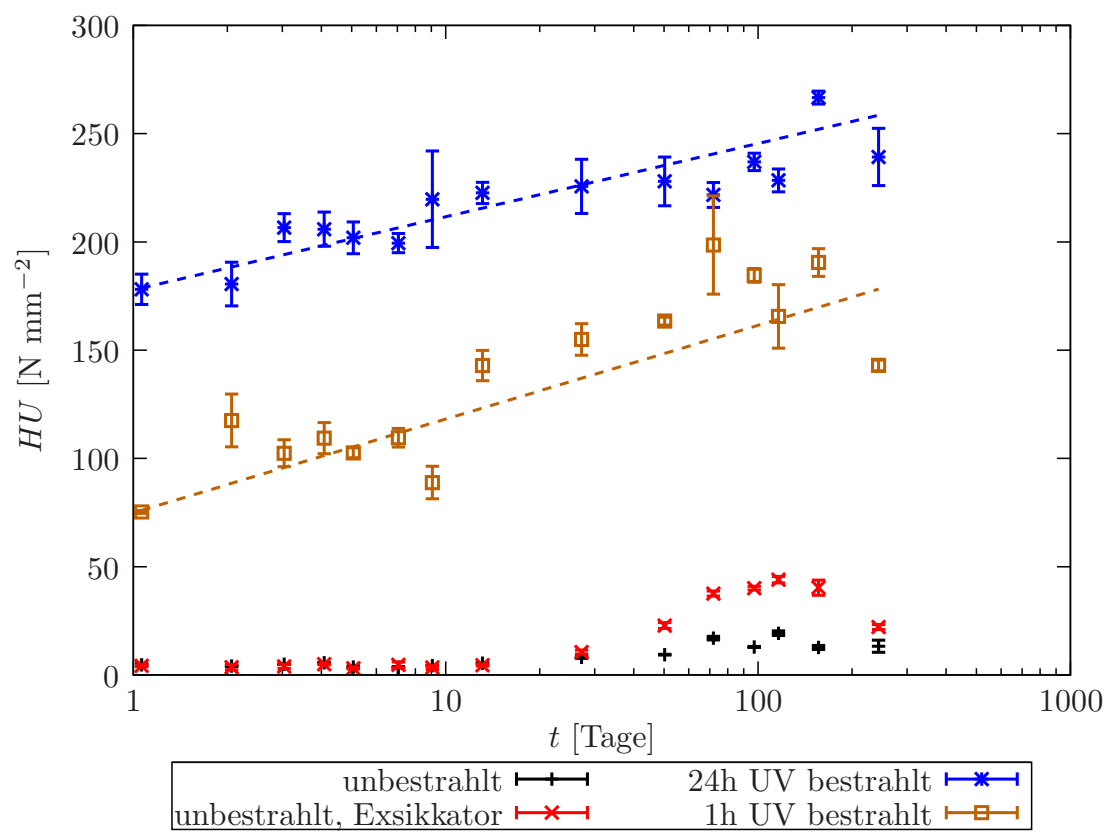

Abbildung 6.6: Langzeitverhalten von unterschiedlich lang bestrahlten Proben und unbestrahlten Proben zum Vergleich. Die Härteänderung wurde hier über viele Monate verfolgt.

Härte besonders stark zunimmt und erst sehr viel später in eine Art Sättigung geht.

Diese lange Reaktionszeit der durch die Bestrahlung frei gewordenen Bindungen liegt darin begründet, dass bei geringer Energieeinstrahlung die dadurch gebildeten Radikale zu weit voneinander getrennt vorliegen und so nicht miteinander vernetzen können. Die Radikale können in Polymeren über sehr lange Zeit - in der Größenordnung von mehreren Tagen oder sogar einigen Monaten - eingeschlossen bleiben und sich entlang der Ketten bewegen [11]. Die Bewegung wird gestoppt, wenn zwei freie Bindungspartner aufeinander treffen und miteinander reagieren können.

\subsection{Modifizierung durch zusätzlichen Vernetzer im Target}

Die laserdeponierten, glatten PMMA-Schichten sind ausgesprochen weich, da bei dem bereits beschriebenen Ablationsprozess die einzelnen Polymerketten stark verkürzt werden, bevor eine Materialdeposition stattfindet. Dies geschieht vornehmlich, da PMMA ein sehr schwacher Absorber ist und für den Materialabtrag aber eine stärkere Bündelung der eingestrahlten Energie im Target benötigt wird. Der Absorptionskoeffizient des Targetmaterials muss also zunächst erhöht werden, wodurch eine Brechung einzelner Bindungen in den Polymerketten zugunsten von Doppel- 


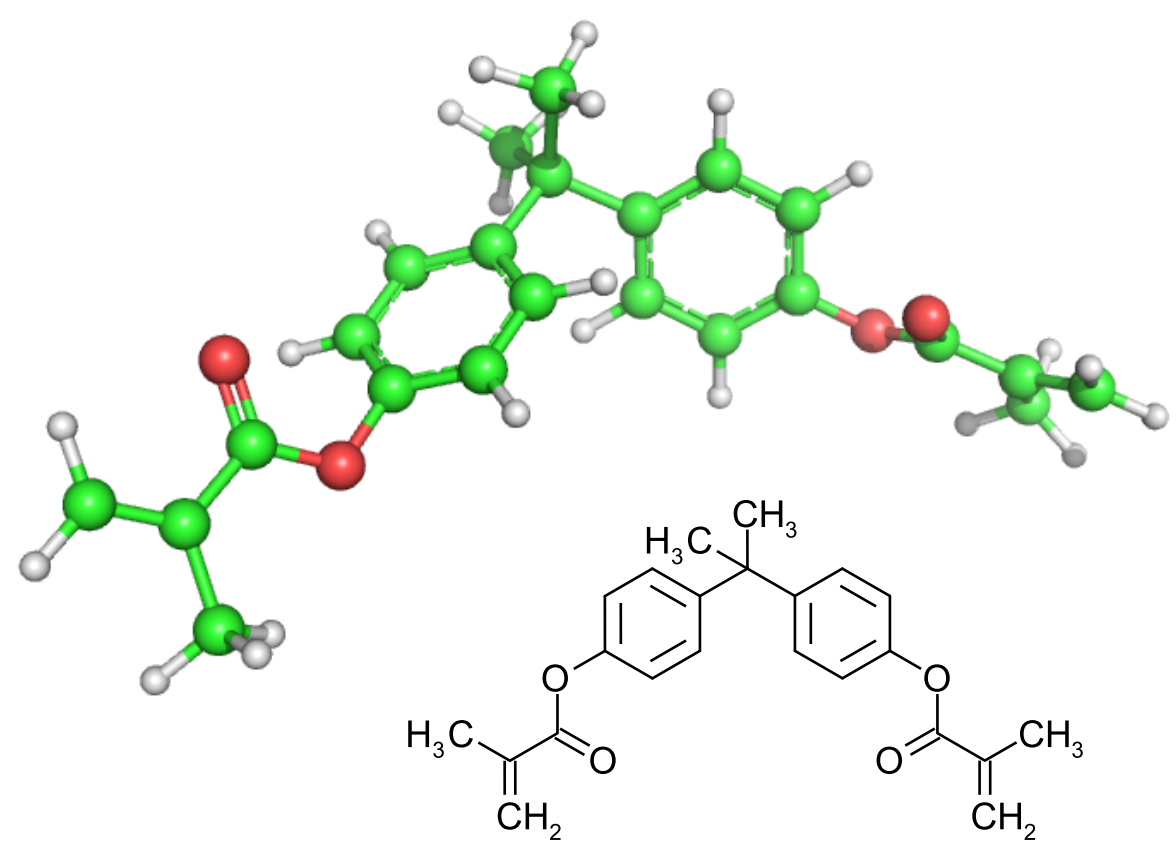

Abbildung 6.7: Visualisierung und Strukturformel von Bisphenol A Dimethacrylat (BisDMA) (C: grün, O: rot, H: weiß)

bindungsbildung zwingend notwendig ist. Hier wird nun beschrieben, wie durch Einbringung einer zweiten Komponente mit hohem Doppelbindungsanteil in das PMMA-Targetmaterial, die Absorption erhöht und damit die Ablation von vornherein begünstigt werden soll. Durch eine stärkere Absorption im Targetmaterial sollte die PMMA-Kettenverkürzung vor der Ablation reduziert werden. Durch die Erhaltung längerer Ketten sollten mehr Verschlaufungs- bzw. Verhakungspunkte in der Polymerschicht erhalten bleiben und die Schichten somit eine größere Härte aufweisen. Diese Beeinflussung der Schichteigenschaft sowie morphologische und chemische Eigenschaften der deponierten Schichten sollen hier untersucht werden.

Als potentieller Vernetzer wurde hier Bisphenol A Dimethacrylat (BisDMA) mit der Summenformel $\mathrm{C}_{23} \mathrm{H}_{24} \mathrm{O}_{4}$ ausgewählt. Wie aus der dazugehörigen Strukturformel (Abb. 6.7) ersichtlich, bietet dieses Material durch seine zwei Phenolringe im Molekül eine hohe Anzahl von $\mathrm{C}=\mathrm{C}$-Doppelbindungen, die dort delokalisiert vorliegen und als Chromophore im Target dienen sollen. Das Bisphenol A ist an zwei Methacrylate gebunden. Dieses Pulver aus sehr kurzen Molekülen mit einer Molmasse von $M_{w}=364,44 \mathrm{~g} \mathrm{~mol}^{-1}$ wird mit dem bereits bekannten PMMA-Pulver vermengt und, wie in Kapitel 2.1.2 beschrieben, zu Targets mit verschiedenen Mengenverhältnissen gepresst. Die Schmelztemperatur der kurzen BisDMA-Moleküle liegt allerdings bereits bei $72-74{ }^{\circ} \mathrm{C}$, weshalb die Temperatur beim Pressen der Targets mit BisDMA-Anteil entsprechend geringer gewählt werden muss. Das ins PMMA-Target eingebrachte Bisphenol A Dimethacrylat (BisDMA) ist nicht nur ein stärkerer Absor- 

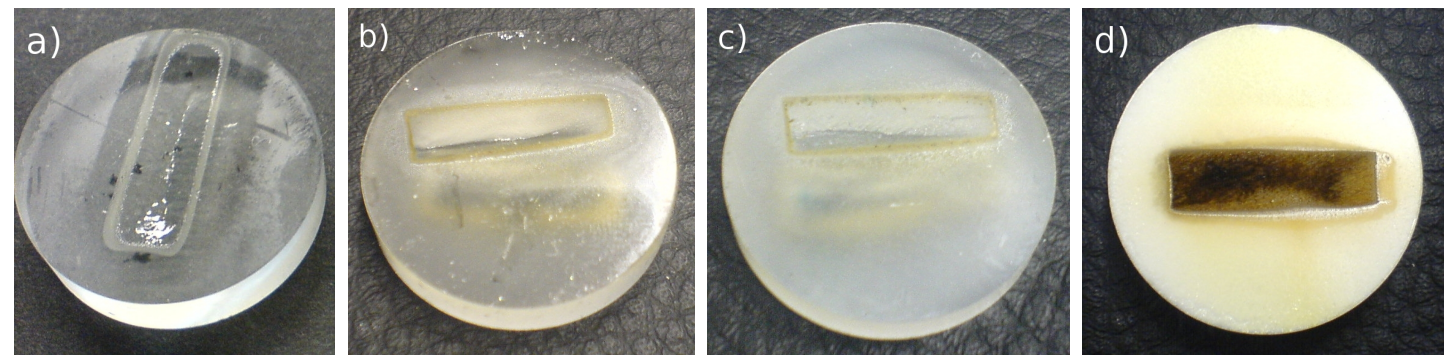

Abbildung 6.8: Fotografien von Targets $(\varnothing=24 \mathrm{~mm})$ nach mehreren Tausend Laserschuss: a) reines PMMA, b) PMMA mit etwa 100 BisDMA/PMMA-Kette (3,7 w\% BisDMA), c) PMMA mit etwa 1.500 BisDMA/PMMA-Kette (36,7 w\% BisDMA), d) reines BisDMA.

ber, sondern dient direkt als Vernetzer zwischen den PMMA-Ketten, was zusätzlich zu einer Härtung der deponierten Schichten führt [106]. Für die Herstellung der Mischschichten wurde wie für die meisten Schichten zuvor eine Laserfluenz von etwa $125 \mathrm{~mJ} \mathrm{~cm}^{-2}$.

\subsubsection{Morphologie}

Im Gegensatz zu den bisher verwendeten gepressten, durchsichtigen PMMA-Target (Abb. 6.8 a) ist ein aus reinem BisDMA gepresstes Target weiß und leicht pulverig (Abb. 6.8d). Durch die Mischung des PMMA- und des BisDMA-Pulvers erhält man also je nach Mengenverhältnis leicht milchige, feste Targets (Abb. 6.8b,c). Nach Beschuss dieser Targets mit mehreren 1.000 Laserpulsen ist um den entstandenen Krater ein gelber Rand zu sehen, der von dem der Ablation vorangegangenen Inkubationsprozess des PMMA herrührt (s. Kap. 4.1). Bei einem reinen BisDMA-Target ist dieser Rand eher schwarz, während die unbeschossene Targetoberfläche neben dem Krater gelblich erscheint. Die an der beschossenen Stelle des Targets ablaufenden Prozesse scheinen sich bei einem solch starken Absorber auf der Oberfläche des Targets auszuweiten. Der Krater selbst weist mit höherem BisDMA-Anteil immer rauere Oberflächen auf. Für reines BisDMA sieht die beschossene Targetoberfläche sogar eher faserig aus und hat sich schwarz verfärbt, was auf einen erhöhten Kohlenstoffanteil nach Beschuss des Targets schließen lässt. Deshalb ist davon auszugehen, dass bei ausreichendem Energieeintrag zunächst die Methacrylatgruppen abgespalten werden und ein Teil der Phenolringe am Target zurück bleibt. Dadurch verringert sich auch die Depositionsrate für reine BisDMA-Schichten mit steigender Pulszahl. Deshalb und wegen der starken Verschmutzung des Lasereintrittsfensters in die UHV-Kammer durch die kleinen BisDMA-Moleküle kann für diese Targets keine konstante Depositionsrate angegeben werden kann.

Entsprechend den nach dem Beschuss rauen Targetoberflächen erkennt man in SEM-Aufnahmen der daraus entstandenen Schichten die Zunahme von Tröpfchen 

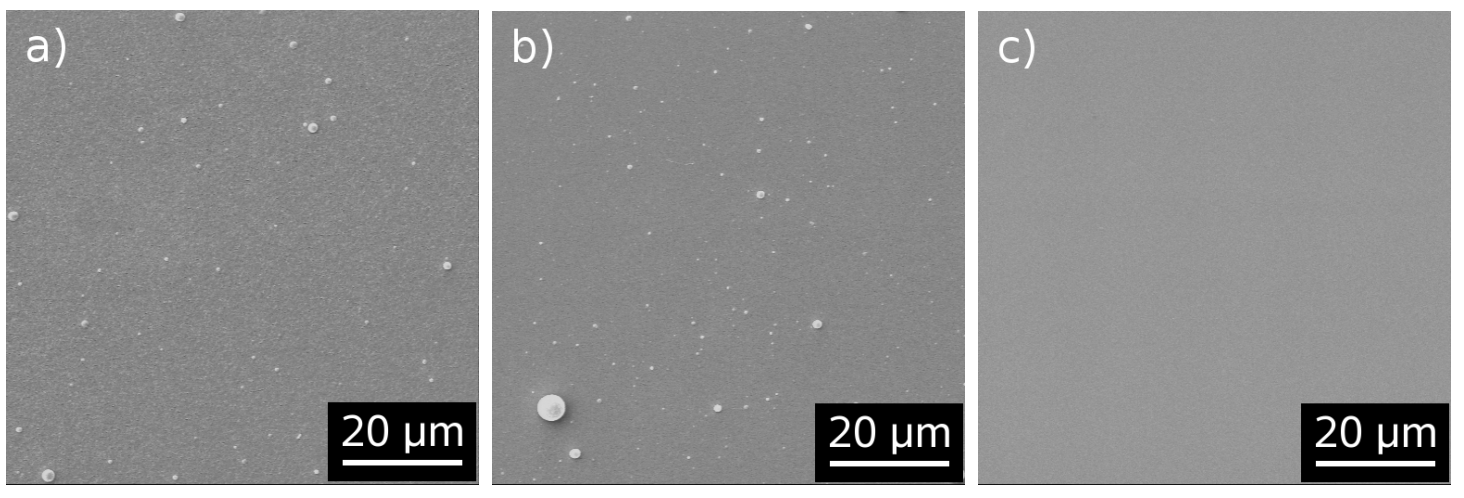

Abbildung 6.9: SEM-Aufnahmen von Schichten hergestellt mit 500 Pulsen bei $125 \mathrm{~mJ} \mathrm{~cm}^{-2}$ mit einem Target aus: a) PMMA mit etwa 100 BisDMA/PMMA-Kette, b) PMMA mit etwa 1.500 BisDMA/PMMA-Kette, c) reinem BisDMA.

mit steigendem BisDMA-Gehalt in den Targets (Abb. 6.9 a,b). Betrachtet man allerdings die aus dem reinen BisDMA entstandene Schicht, ist diese wieder vollkommen tröpfchenfrei (Abb. 6.9c). Die Tröpfchen auf den Mischschichten sind also durch das BisDMA induzierte PMMA-Tröpfchen. Das BisDMA im Target erhöht somit die Absorption im Material so stark, dass wieder eine Überhitzung im Targetinneren stattfindet, wie sie bereits für reine PMMA-Targets unter hoher Laserfluenzeinstrahlung diskutiert wurde (s. Kap. 3.3). Diese lokale Überhitzung spricht dafür, dass das BisDMA nur unzureichend mit dem PMMA vermengt wurde. Eine Möglichkeit, diese lokale Überhitzung zu vermeiden, könnte darin bestehen, das PMMA selbst zu modifizieren, indem an die PMMA-Kettenenden jeweils direkt die absorbierenden Phenolringe gehängt werden. Dies könnte z. B. mit Hilfe des reversiblen Additions-Fragmentierungs-Kettenübertragungs (reversible addition-fragmentation chain transfer, RAFT) realisiert werden [74, 10].

Trotz der nun nicht mehr vollkommen glatten Schichten, soll untersucht werden, ob dieser Ansatz der Schichthärtung positive Ergebnisse liefert. Dazu betrachten wir uns außerdem die Charakteristika des reinen BisDMA noch genauer. Im Vergleich zu der Profilmessung einer reinen PMMA-Schicht, bei der die Oberfläche aufgrund der Affinität zu den Klemmbacken an den Seiten leichte Spitzen aufwies (s. Abb. 4.5), erkennt man bei einer reinen BisDMA-Schicht, dass sich die Oberfläche an den Seiten, wo das Substrat mit Backen geklemmt war, abrundet (Abb. 6.10). Dieses Schichtmaterial weist also eine größere Oberflächenspannung als das PMMA auf. Die Schichtdicke ergibt sich nach 75.000 Pulsen zu 5,5 $\mu \mathrm{m}$, was lediglich einer mittleren Depositionsrate von $0,07 \mathrm{~nm} /$ Puls entspricht. Da allerdings der Druck in der PLD-Kammer - anstatt den üblichen Werten während der Polymerdeposition (im Bereich von $10^{-6}$ mbar) - gegen Ende des Depositionsprozesses schon wieder Ultrahochvakuum erreicht hatte (im Bereich von $10^{-8}$ mbar), hat zu dieser Zeit wohl 


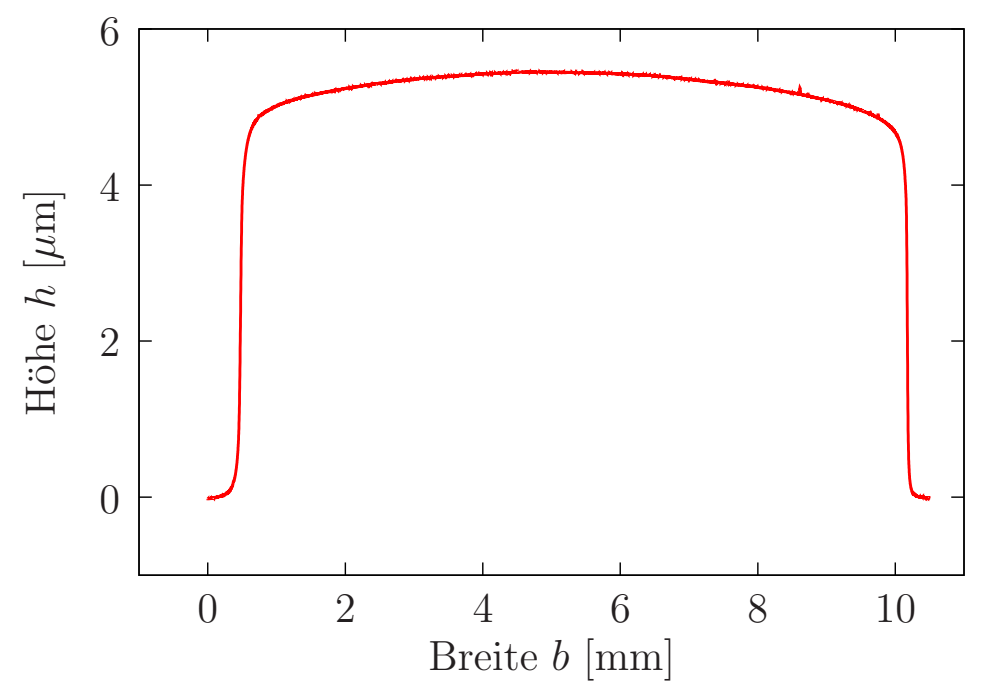

Abbildung 6.10: Schichtdickenverteilung aus Profilometermessungen: Profil einer $1 \mathrm{~cm}$ breiten BisDMA-Schicht mit 75.000 Pulsen bei $125 \mathrm{~mJ} \mathrm{~cm}^{-2}$ hergestellt. Es wird dabei eine Schichtdicke von etwa $5,5 \mu \mathrm{m}$ erreicht. Der $\cos ^{n} \varphi$-Fit ergibt für diese reine BisDMASchicht einen Exponenten von $n=21$.

kaum noch ein Übertrag stattgefunden. Die Rate zu Beginn der Schichtdeposition lag also um einiges höher. Das starke Absinken der Depositionsrate ist zum einen auf eine immense Verschmutzung des Lasereintrittsfensters durch den hohen Abtrag der sehr kurzen BisDMA-Moleküle, die sich in der ganzen Kammer niederschlagen, zurückzuführen, zum anderen führen die Laserpulse direkt am Target schon zu einer starken Veränderung des Materials z. B. zu einer Vernetzung oder zu einem starken Anstieg des Kohlenstoffanteils, wodurch weniger Material ablatiert werden kann. Im Vergleich zu den glatten PMMA-Schichten $(n=30)$ ist hier die Bündelung des BisDMA bei der Deposition etwas geringer. Der $\cos ^{n} \varphi$-Fit ergibt für reine BisDMASchichten einen Exponenten von $n=21$.

\subsubsection{Chemische Eigenschaften}

Um Rückschlüsse auf den Einfluss des BisDMA auf das PMMA ziehen zu können, wurden SEC-Messungen von deponierten Schichten aus den verschiedenen Mischtargets mit einer Messung einer reinen PMMA-Schicht verglichen (Abb. 6.11). Durch Zugabe von 3,7 w\% BisDMA zum PMMA im Target (etwa 100 BisDMA-Moleküle pro PMMA-Kette) ist gegenüber einer deponierten Schicht aus reinem PMMA mit $\bar{M}_{w}=2.900 \mathrm{~g} \mathrm{~mol}^{-1}$ ein Anstieg der mittleren Molmasse auf $\bar{M}_{w}=4.400 \mathrm{~g} \mathrm{~mol}^{-1} \mathrm{zu}$ bemerken. Zusätzlich taucht bei kleinen Molmassen ein Peak auf, der sich aus den BisDMA-Molekülen ergibt, die durch den Depositionsprozess direkt auf die Schicht 


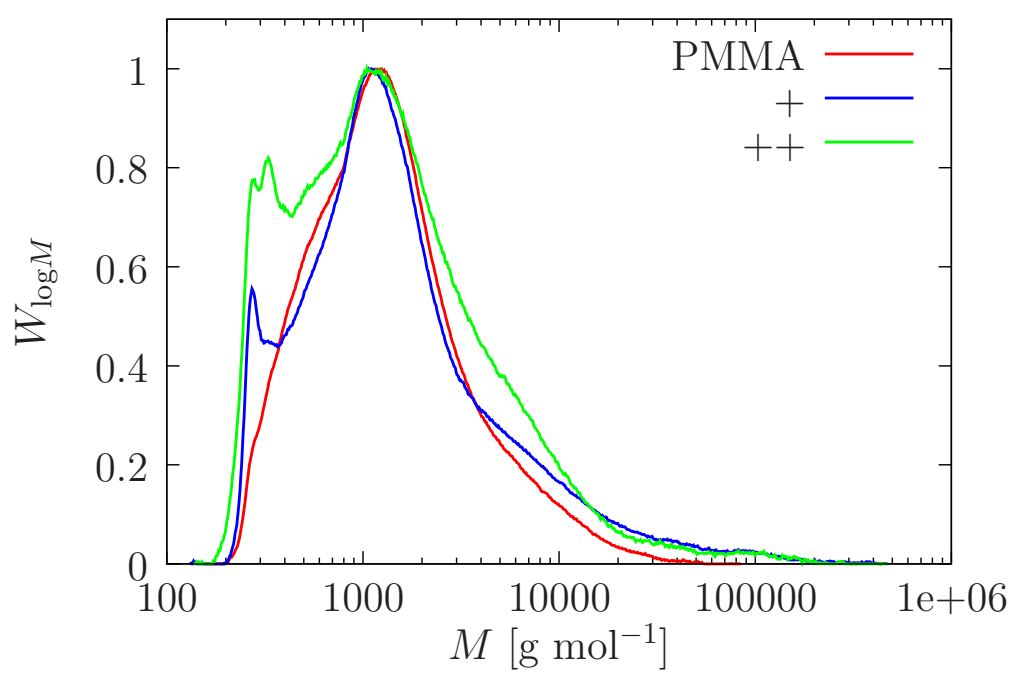

Abbildung 6.11: SEC-Messungen von Schichten mit BisDMA zu verschiedenen Anteilen im PMMA-Target hergestellt bei $130 \mathrm{~mJ} \mathrm{~cm}^{-2}$. Die mittlere Molmasse von $\bar{M}_{w}=$ $2.900 \mathrm{~g} \mathrm{~mol}^{-1}$ (Polydispersität von $D=3,5$ ) für reine PMMA-Schichten wird durch Zugabe von BisDMA im Target (+: $3,7 \mathrm{w} \%$ BisDMA) erst erhöht auf $\bar{M}_{w}=4.400 \mathrm{~g} \mathrm{~mol}^{-1}$ $(D=4,8)$ und sinkt bei noch höherem Anteil des Vernetzers (++: $36,7 \mathrm{w} \%$ BisDMA) mit sehr geringer eigener Molmasse wieder auf $\bar{M}_{w}=3.500 \mathrm{~g} \mathrm{~mol}^{-1}(D=4,3)$.

deponiert wurden, ohne mit den PMMA-Ketten zu vernetzen. Durch diesen Peak bei kleinen Molmassen und der sich ausbildenden Schulter bei größeren Molmassen nimmt entsprechend die Polydispersität der Probe von $D=3,5$ für eine reine PMMA-Schicht auf nun $D=4,8 \mathrm{zu}$. Durch einen höheren Anteil des Vernetzers im Target von 36,7 w\% (etwa 1.500 BisDMA-Moleküle pro PMMA-Kette) erhöht sich in den SEC-Messungen auch der Peak, der sich direkt aus den BisDMA-Molekülen ergibt. Das Target scheint mit BisDMA übersättigt zu sein. Das BisDMA wird in größerer Anzahl einfach übertragen, was aus dem Anstieg des vorderen Peaks zu sehen ist. Außerdem führt eine höhere Anzahl von BisDMA-Molekülen dazu, dass die Vernetzung der PMMA-Ketten dichter stattfindet und so die gleiche Länge der Ketten in den SEC-Messungen nun als geringere Molmasse detektiert wird, wodurch der Vergleich der mittleren Molmassen fehlerbehafteter wird. Bei einer zu hohen Anzahl von BisDMA-Molekülen können diese auch verstärkt miteinander reagieren, anstatt die PMMA-Ketten miteinander zu verbinden. Sehr hohe Molmassen werden nun weniger detektiert. Damit ergibt sich wieder eine geringere mittlere Molmasse von $\bar{M}_{w}=3.500 \mathrm{~g} \mathrm{~mol}^{-1}$ mit einer geringeren Polydispersität von $D=4,3$. 


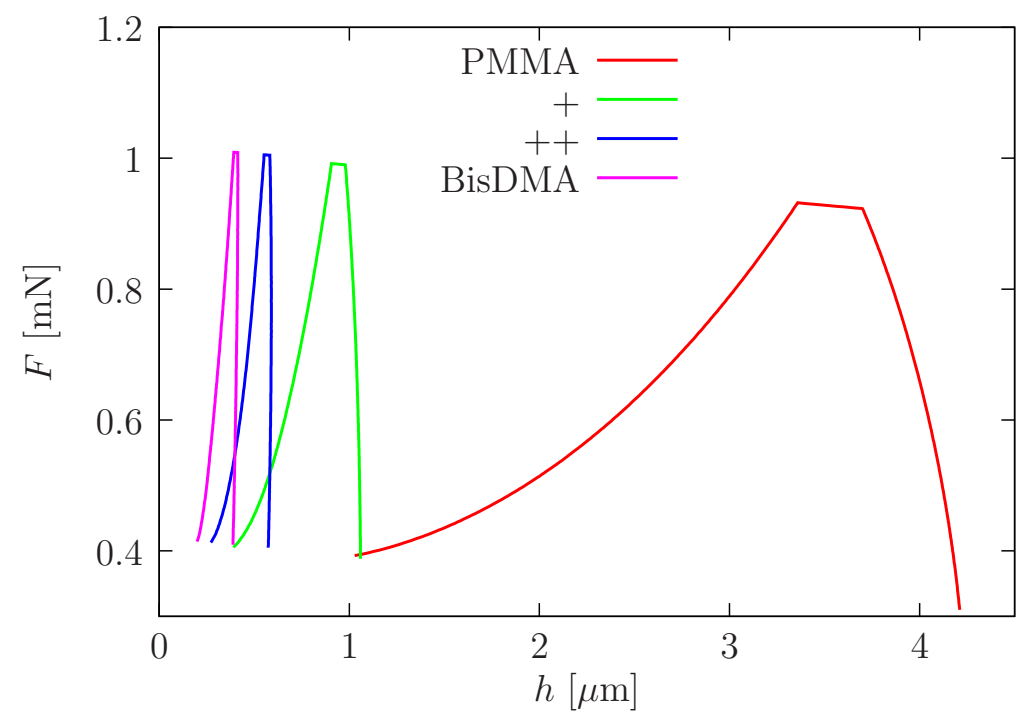

Abbildung 6.12: Kraft-Eindringtiefe-Kurven von PMMA/BisDMA-Schichten mit verschiedenen anteiligen Zusammensetzungen hergestellt mit je 30.000 Pulsen bei $125 \mathrm{~mJ} \mathrm{~cm}^{-2}$. Es ergibt sich aus den Messungen für diese Schichten: $H U_{+}=(37 \pm 2) \mathrm{N} \mathrm{mm}^{-2}, H U_{++}=$ $(95,7 \pm 0,5) \mathrm{N} \mathrm{mm}^{-2}, H U_{\text {BisDMA }}=(180 \pm 8) \mathrm{N} \mathrm{mm}^{-2}$.

\subsubsection{Mechanische Eigenschaften}

Dass das BisDMA größere Auswirkungen auf die deponierten Schichten hat, spiegelt sich auch in den Härtemessungen wieder. Aus dem Verlauf der Kraft-EindringtiefeKurven ist die starke Reduktion der Eindringtiefe bei gleicher vorgegebener Maximalkraft zu erkennen (Abb.6.12), wodurch direkt eine Erhöhung der Universalhärte beschrieben wird. Zum Vergleich ist auch der Kurvenverlauf einer reinen PMMASchicht mit einer Universalhärte von $H U=2,8 \pm 0,2 \mathrm{~N} \mathrm{~mm}^{-2}$ eingetragen (s. auch Abb. 4.9.

Der Anstieg der Universalhärte mit steigender BisDMA-Massenprozentzahl im PMMA-Mischtarget ist in Abbildung 6.13 zu sehen. Eine Schicht, hergestellt aus reinem BisDMA, hat eine Universalhärte von $H U=180 \pm 8 \mathrm{~N} \mathrm{~mm}^{-2}$, während Schichten aus den Mischtargets auf Universalhärten von $H U=37 \pm 2 \mathrm{~N} \mathrm{~mm}^{-2}$ für $3,7 \mathrm{w} \%$ BisDMA bzw. von $H U=95,7 \pm 0,5 \mathrm{~N} \mathrm{~mm}^{-2}$ für 36,7 w\% BisDMA kommen. Die härtesten Schichten werden also bei einer Ablation von reinen BisDMA-Targets erreicht. Mit Veränderung des BisDMA-Anteils im PMMA-Target lässt sich der Härtegrad der deponierten Schichten also kontinuierlich variieren. Allerdings liegen die so erreichten Härtewerte unter den Härtewerten, wie sie durch UV-Bestrahlung erreicht und in Kapitel 6.1.3 bereits beschrieben wurden. 


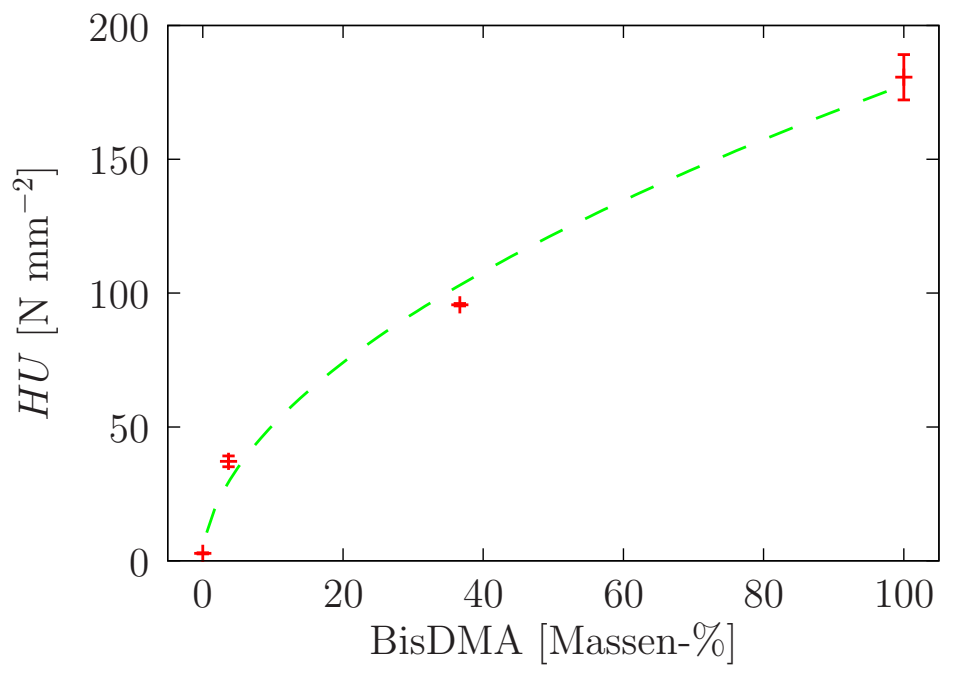

Abbildung 6.13: Auftragung der sich aus den Kraft-Eindringtiefe-Kurven aus Abb. 6.12 ergebenden Universalhärten gegen die prozentuale Zusammensetzung von PMMA/BisDMA des jeweiligen Targets.

\subsection{Modifizierung durch thermische Behandlung}

Nachdem in Kapitel 5 schon die thermische Stabilität der glatten PMMA-Schichten vor allem in Bezug auf die Glasübergangstemperatur, die chemischen Veränderungen und die abdampfenden Bestandteile diskutiert wurden, soll nun näher darauf eingegangen werden, welche Folgen diese thermische Behandlung auf die Morphologie der PMMA-Schichten hat und wie sich die mechanischen Eigenschaften in Form der Härte durch diese thermische Behandlung ändern.

\subsubsection{Morphologie}

Durch den thermischen Einfluss spielt das Substrat, auf dem die PMMA-Schicht deponiert wurde, nun eine bedeutende Rolle. Bei höheren Temperaturen werden Atome und damit auch Moleküle und ganze Ketten beweglicher, die Diffusion auf der Substratoberfläche nimmt zu. Das Bestreben des PMMA von Oberflächen zu entnetzen, wird damit bei hohen Temperaturen begünstigt [83. Ebenso ist bekannt, dass dünne Schichten durch ihr ungünstiges Oberfläche-zu-Volumen-Verhältnis leichter entnetzen als dickere [89]. Beide Merkmale zusammen spielen also eine wichtige Rolle bei der Untersuchung sehr dünner PMMA-Schichten.

Die vollständige Entnetzung z. B. einer etwa $100 \mathrm{~nm}$ dicken PMMA-Schicht bei $200^{\circ} \mathrm{C}$ ist besonders gut lichtmikroskopisch zu erfassen, da hier das blanke Siliziumsubstrat stark reflektiert (Abb. 6.14b). Die Schicht hat sich vollständig zu runden Tröpfchen zusammengezogen. Durch den Farbverlauf in den Tröpfchen ist die kugel- 

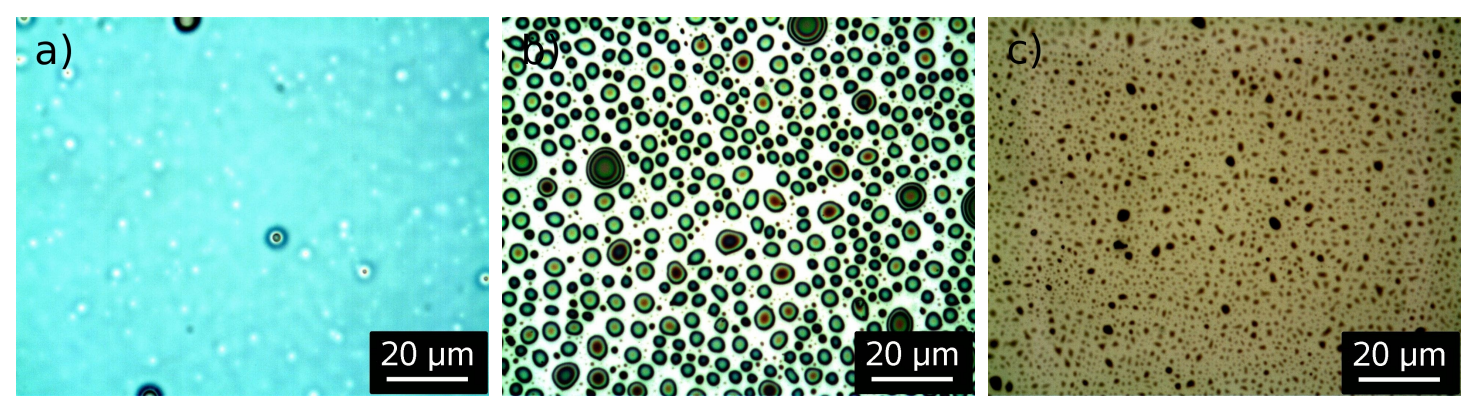

Abbildung 6.14: Lichtmikroskopische Aufnahmen von PMMA-Schichten hergestellt mit 500 Pulsen bei $125 \mathrm{~mJ} \mathrm{~cm}^{-2}$ bei a) Raumtemperatur, b) $200^{\circ} \mathrm{C}$, c) $260^{\circ} \mathrm{C}$.
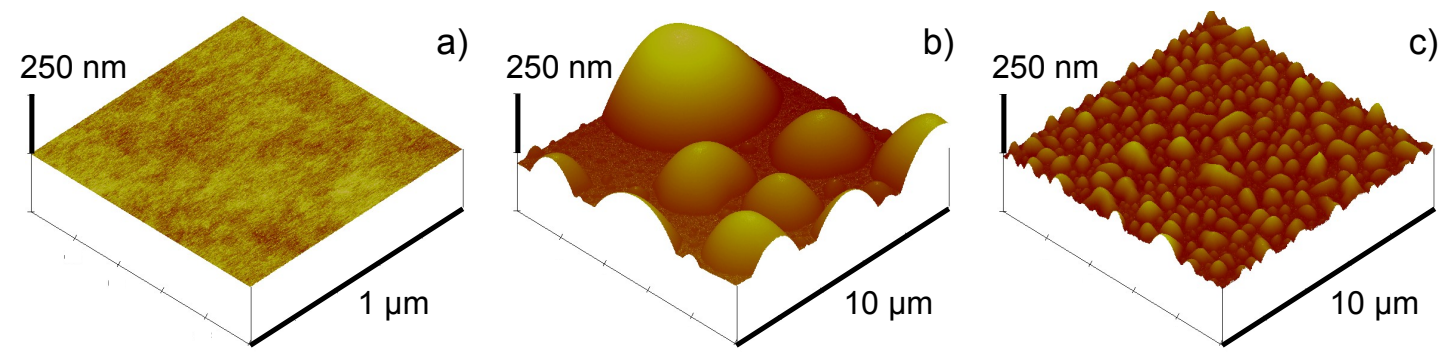

Abbildung 6.15: AFM-Bilder von PMMA-Schichten hergestellt mit 500 Pulsen bei $125 \mathrm{~mJ} \mathrm{~cm}^{-2}$ bei a) Raumtemperatur mit einer RMS-Rauigkeit von $\sigma_{\mathrm{RMS}}=0,3 \mathrm{~nm}, \mathrm{~b}$ ) $200{ }^{\circ} \mathrm{C}$ mit $\left.\sigma_{\mathrm{RMS}}=67,4 \mathrm{~nm}, \mathrm{c}\right) 260^{\circ} \mathrm{C}$ mit $\sigma_{\mathrm{RMS}}=14,4 \mathrm{~nm}$.

artige Form gut zu erkennen. Im Vergleich dazu sieht man bei einer bei Raumtemperatur deponierten Schicht hauptsächlich Dreckpartikel, die sich unter anderem an Luft auf der Schicht abgelagert haben bzw. Staub auf dem Objektiv des Mikroskops (Abb. 6.14a). Betrachtet man dagegen bei $260^{\circ} \mathrm{C}$ deponiertes Material, ist weder eine intakte Schicht noch größere Tröpfchen zu erkennen (Abb.6.14 c). Die Tröpfchen haben sich zersetzt. Die Vorgänge bei der thermischen Zersetzung wurden bereits in Kapitel 5.4 diskutiert.

Eine genauere Betrachtung der Schichten im AFM zeigt für einen $1 \times 1 \mu \mathrm{m}^{2}$ großen Ausschnitt einer bei Raumtemperatur deponierten Schicht die bereits in Kapitel 3.3 gezeigte, glatte Oberflächenbeschaffenheit mit einer RMS-Rauigkeit von $\sigma_{\mathrm{RMS}}=0,3 \mathrm{~nm}$ (Abb. 6.15a). Deponiert man die Schichten bei höheren Temperaturen, kann man die resultierenden Schichten besser für größere Ausschnitte (hier $10 \times 10 \mu \mathrm{m}^{2}$ ) betrachten, da dies repräsentativere Ergebnisse liefert. So ist für Schichten, die bei $200{ }^{\circ} \mathrm{C}$ deponiert wurden, selbst bei einer angestrebten Dicke von etwa $100 \mathrm{~nm}$ noch eine totale Entnetzung vom Si-Substrat zu bemerken (Abb. 6.15b). Die sich ergebenden Tröpfchen erreichen lateral Durchmesser von mehreren $\mu \mathrm{m}$ und Höhen von bis zu $250 \mathrm{~nm}$. Durch diese hohen und so unterschiedlichen Strukturen ist kein sinnvoller Vergleich der RMS-Raugkeiten mehr möglich. Will man die Werte trotzdem angeben, würde sich z. B. für den hier betrachteten Ausschnitt eine RMS- 

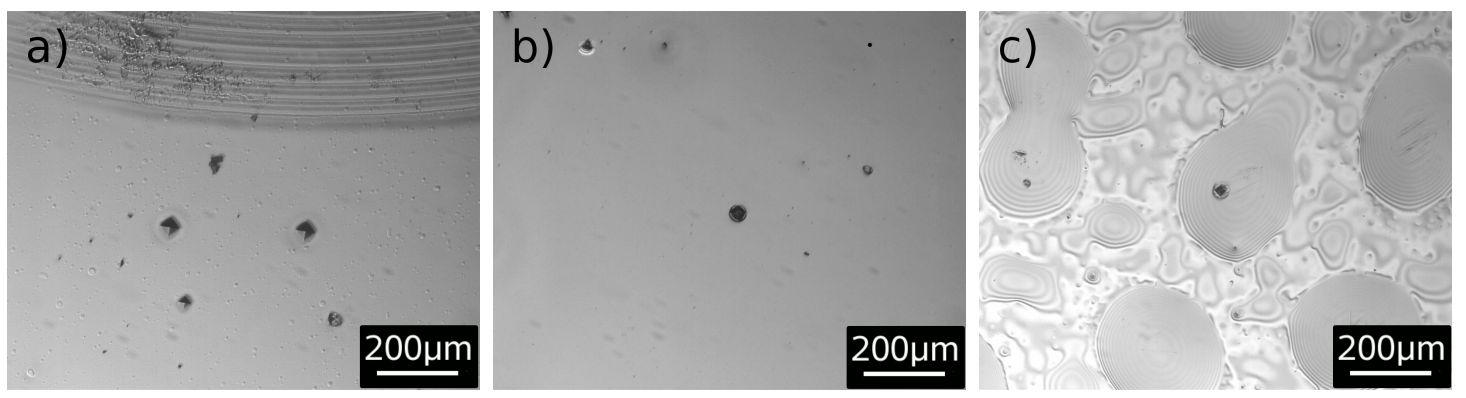

Abbildung 6.16: Lichtmikroskopische Aufnahmen von dicken PMMA-Schichten hergestellt mit 100.000 Pulsen bei $125 \mathrm{~mJ} \mathrm{~cm}^{-2}$ bei a) Raumtemperatur und bei einer Substrattemperatur von b) $T_{s}=200^{\circ} \mathrm{C}$ und c) $T_{s}=260^{\circ} \mathrm{C}$.

Rauigkeit von $\sigma_{\mathrm{RMS}}=67,4 \mathrm{~nm}$ ergeben. Wie schon aus den lichtmikroskopischen Aufnahmen zu sehen war, sind bei einer Deposition bei $260^{\circ} \mathrm{C}$ weder eine intakte Schicht noch größere Tröpfchen zu erkennen (Abb. 6.15c). Die Auswertung der Rauigkeit ergibt hier einen Wert von $\sigma_{\mathrm{RMS}}=14,4 \mathrm{~nm}$.

Betrachtet man dagegen sehr dicke PMMA-Schichten im Lichtmikroskop, ist im Vergleich mit den dünnen Schichten aus Abbildung 6.14 vor allem eine Veränderung der Oberfläche der bei einer Substrattemperatur von $T_{s}=200{ }^{\circ} \mathrm{C}$ hergestellten Schicht zu bemerken (Abb. 6.16b). Die Schicht ist nicht mehr entnetzt, sondern das Substrat wird durch die nun sehr große Menge an deponiertem Material vollständig bedeckt. Die Schicht hat auch bei diesen hohen Temperaturen keine Möglichkeit mehr, vom Substrat zu entnetzen. Die sehr dicke Schicht, die bei Raumtemperatur hergestellt wurde, ist nach wie vor glatt (Abb. 6.16a). Dass die RT-Schichten mit zunehmender Schichtdicke nicht aufrauen, wurde bereits in Kapitel 4.2 gezeigt. Nach einer Schichtherstellung bei $T_{s}=260^{\circ} \mathrm{C}$ hat sich durch die Menge an deponiertem Material nicht die ganze Schicht zersetzt (Abb. 6.16c). Einige intakte Bereiche, die durch ihre runde Form an die entnetzten Tröpfchen erinnern, sind noch durch ihre gleichmäßigen Schichtdickenringe zu erkennen. Die degradierten Bereiche der Schicht wirken dagegen ungleichmäßig und ölig.

Die Eindrücke, die in diesen Aufnahmen zu sehen sind, stammen von Härtemessungen, die hier im Anschluss in Kapitel 6.3 diskutiert werden sollen. Die Rillen am oberen Rand der Abbildung 6.16a stammen vom Auflagering der Messapparatur. Dies ist wieder eine Bestätigung dafür, wie weich die Schichten nach einer Deposition bei Raumtemperatur sind.

Betrachtet man makroskopisch die Oberfläche einer dicken Schicht wie z. B. den Querschnitt über die ganze Probe einer bei $230^{\circ} \mathrm{C}$ hergestellten Schicht, so sind größere wellige Strukturen zu erkennen, die sich durch den thermischen Einfluss auf das Material gebildet haben (Abb. 6.17). Außerdem ist auf der linken Seite der Auftragung ein ausgeprägter Peak zu sehen. Diese Seite der Schicht zeigte bei der 


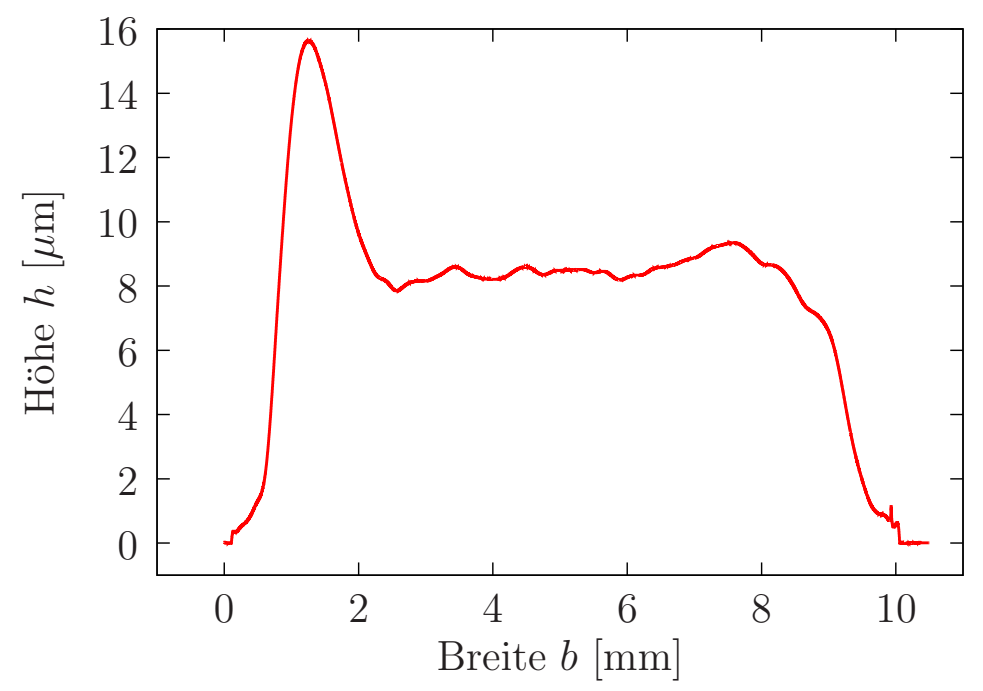

Abbildung 6.17: Schichtdickenverteilung aus Profilometermessungen einer $1 \mathrm{~cm}$ breiten PMMA-Schicht mit 100.000 Pulsen bei $125 \mathrm{~mJ} \mathrm{~cm}^{-2}$ hergestellt und nachträglich bei $230^{\circ} \mathrm{C}$ ausgelagert. Es wird dabei eine Schichtdicke von etwa $8,5 \mu \mathrm{m}$ erreicht. Durch die Auslagerung gleitet die Schicht zur unteren Klemmbacke (linke Seite in der Abbildung).

Deposition im Ofen nach unten, so dass das PMMA auf die dort angebrachte Klemmbacke geflossen ist. Auf kleinem Maßstab sind diese großen Oberflächenstrukturen aber kaum merklich, da sich kleine Unebenheiten in diesem Fließzustand eher glatt ziehen. Außerdem ergibt sich hier die erreichte Schichtdicke in der Substratmitte bei einer Deposition mit 100.000 Pulsen zu etwa 8,5 $\mu \mathrm{m}$. Damit liegt die Schichtdicke um circa $40 \%$ niedriger als bei einer PMMA-Schicht, die bei Raumtemperatur deponiert wurde (s. Abb. 4.5). Damit wird der Schichtdickenverlust, der thermogravimetrisch (nach etwa $2 \mathrm{~h}$ hochheizen) bestimmt wurde (s. Kap. 5.4), hier auch direkt für die Laserdeposition bei hohen Temperaturen verifiziert. Hierbei dauert die Schichtdeposition auf ein (geheiztes) Substrat etwa 7 h. Der Schichtdickenverlust scheint somit nicht zeitsensitiv, da unabhängig von der Dauer des thermischen Einflusses die Schichten die gleiche Menge an Material verloren haben.

\subsubsection{Mechanische Eigenschaften}

Eine weitere bedeutende Veränderung der PMMA-Schichten durch thermischen Einfluss findet man bei den mechanischen Eigenschaften. So erreichen die zuvor sehr weichen PMMA-Schichten (s. Kap. 4.5) durch thermische Behandlung bei $200^{\circ} \mathrm{C}$ während der Deposition eine Universalhärte von etwa $H U=200 \mathrm{~N} \mathrm{~mm}^{-2}$ [31]. Besonders erwähnenswert ist hier, dass es für die Ergebnisse der Härtemessungen keinen Unterschied macht, ob die Schichten bei einer bestimmten Substrattemperatur de- 


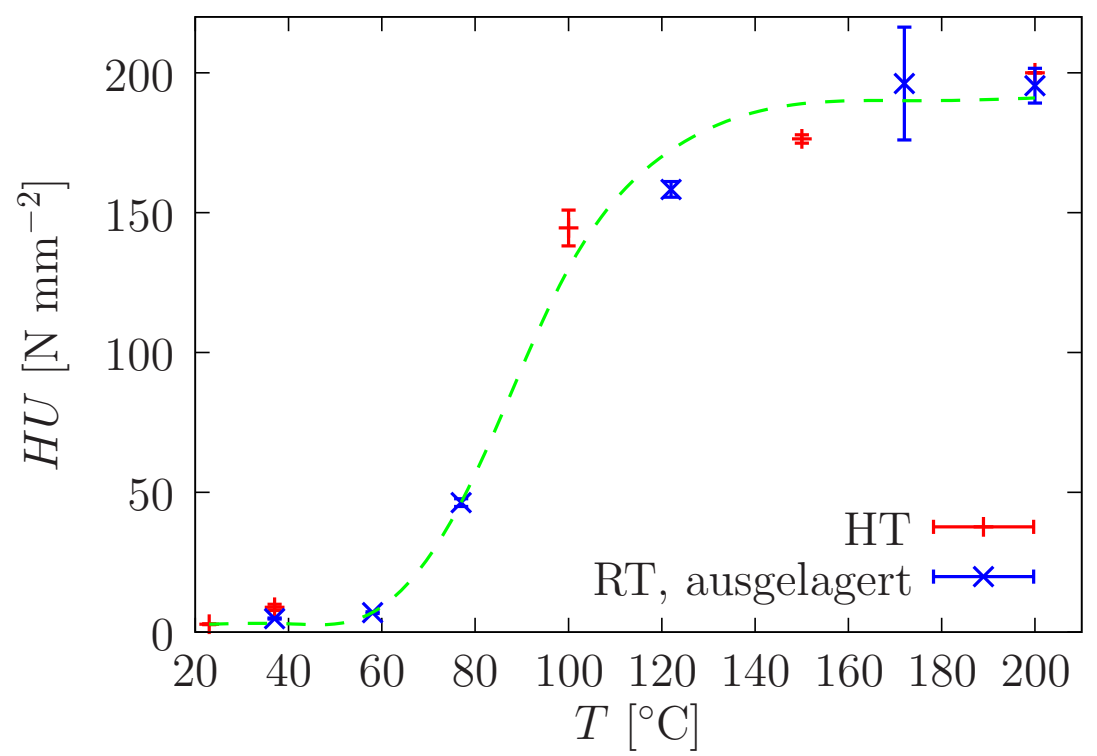

Abbildung 6.18: Härteänderung durch thermische Behandlung während und nach der Deposition der PMMA-Schichten

poniert oder bei Raumtemperatur deponiert und anschließend ausgelagert wurden. Die Härteuntersuchungen der noch intakten Bereiche einer bei $260^{\circ} \mathrm{C}$ hergestellten Schicht ergaben etwa $H U=196 \mathrm{~N} \mathrm{~mm}^{-2}$, während degradierte Bereiche eine Härte von $H U=496 \mathrm{~N} \mathrm{~mm}^{-2}$ aufweisen (Abb. 6.16 c). Diese sind aufgrund ihrer unregelmäßigen Oberflächenbeschaffenheit (s. Abb.6.16 c) uninteressant. Da die degradierten Bereiche nicht weiter betrachtet werden und die intakten Bereiche gegenüber einer bei $200^{\circ} \mathrm{C}$ hergestellten Schicht nicht mehr an Härte zunehmen, wird hier nun nur noch der Härteverlauf von Raumtemperatur bis $200^{\circ} \mathrm{C}$ betrachtet (Abb. 6.18).

Erhöht man die Substrattemperatur für die Schichtdeposition nur leicht, bleibt die Härte zunächst konstant. Aber bereits kurz oberhalb von $60^{\circ} \mathrm{C}$ - also bereits unterhalb der Glasübergangstemperatur - steigt die Universalhärte an. Oberhalb von $150{ }^{\circ} \mathrm{C}$ bleibt die Härte der Schichten dann konstant bei etwa $H U=200 \mathrm{~N} \mathrm{~mm}^{-2}$. Eine Nachhärtung der Schichten durch längere Wartezeiten zwischen Modifizierung und Härtemessung, wie sie für die UV-bestrahlten Schichten diskutiert wurde (s. Kap. 6.1.3), konnte für die thermisch behandelten Schichten nicht festgestellt werden.

Damit ist nicht nur der Materialverlust bei thermischer Behandlung zeitunabhängig, sondern auch die resultierende Härte hängt nur von der Temperatur ab und nicht von der Zeit, in der die Schicht dieser Temperatur ausgesetzt war. Für Härtemessungen verwendet man sehr dicke Schichten, um den Einfluss des Substrats auszuschließen. Für solche Schichten dauert die Deposition (mit 100.000 Pulsen) etwa $7 \mathrm{~h}$. Die Schicht wäre also bei einer Hochtemperatur-Deposition für $7 \mathrm{~h}$ einer er- 
höhten Temperatur ausgesetzt. Die nachträgliche Auslagerung dagegen wurde meist nach etwa 2 Stunden beendet. Trotz dieser verschieden langen Temperatureinflüsse ergeben sich gleiche Universalhärten für die thermische Behandlung während der Deposition und für eine nachträgliche Auslagerung von deponierten Schichten bei Raumtemperatur.

\subsection{Fazit}

Während der Bestrahlung eines Polymers treten gleichzeitig Vernetzung und Spaltung auf, wobei die Gewichtung von Vernetzung (Gelbildung) zu Spaltung (Degradation) von der Struktur des Polymers abhängt [63, 64. In dieser Arbeit kann die Spaltung der PMMA-Ketten direkt anhand der geringeren Molmassen aus SECMessungen gezeigt werden. Auf den Vernetzungsgrad des Polymers kann dagegen über die Härteänderungen des Materials geschlossen werden [62]. Allgemein lässt sich festhalten, dass Polymere mit quaternären Kohlenstoffatomen - also Kohlenstoffatomen, die über Einfachbindungen mit vier weiteren Kohlenstoffatomen verbunden sind - mit ihren langen anhängenden Gruppen wie der Estergruppe beim PMMA eher zur Spaltung neigen, während Polymere, die wenigstens ein Wasserstoffatom an jedem Kohlenstoffatom der Hauptkette gebunden haben, eher zur Vernetzung neigen [11. Dabei wird davon ausgegangen, dass Gelbildung auftritt, wenn wenigstens eine vernetzende Einheit pro zwei Molekülen vorhanden ist. Dagegen wird die Tendenz zur Spaltung von Polymeren mit quaternären Kohlenstoffatomen einer räumlichen Behinderung durch die anhängenden Gruppen zugeschrieben. Durch diese Seitengruppen wird die Kettenbewegung eingeschränkt und damit Vernetzung erschwert. PMMA wird eigentlich als nicht-gelbildendes Polymer angesehen, also als Polymer, welches nach Bestrahlung degradiert und damit in Lösungsmitteln löslicher wird.

HALL zeigte allerdings, dass sich die Löslichkeit von PMMA als Funktion der Fluenz ändert; wie erwartet, steigt zunächst die Löslichkeit von PMMA mit steigender Fluenz an, mit weiter ansteigender Fluenz sinkt sie aber wieder ab [42, 43]. Für die Anwendung in der Lithographie bedeutet dies also, dass sich PMMA für hohe Fluenzen von einem positiven Photolack (auflösender Typus) in einen negativen Photolack (nicht-auflösender Typus) umwandelt. Diese Fluenzabhängigkeit ist auch die Erklärung dafür, dass die glatte Komponente der Tröpfchenschichten vernetzt ist und unsere bei niedrigen Fluenzen hergestellten Schichten so weich, also nicht vernetzt sind. LEE beschreibt die Vernetzung und Spaltung von PMMA für einen linearen Energietransfer (LET, linear energy transfer) mit MeV-Elektronenstrahl oder $\mathrm{MeV}-\mathrm{He}^{+}$- oder $\mathrm{Ar}^{+}$-Teilchen [64]. Dabei ist bei niedrigem LET die Wahrscheinlichkeit, Radikalpaare für eine Vernetzung von zwei benachbarten Polymerketten zu erzeugen, sehr gering. Allerdings kann die absorbierte Energie entlang der Ketten wandern und sich überlappen, wodurch eine hinreichende Energie resultiert, um die Bindung in einer Kette zu brechen. Ein hoher LET produziert also wahrscheinlich 
eine hohe Konzentration freier Radikale über mehrere benachbarte Polymerketten, wodurch in diesem Fall eine Überschneidung der Teilchenspuren möglich ist und die Vernetzung gegenüber der Spaltung erhöht ist. Dagegen wirkt ein niedriger LET lediglich auf eine einzelne Kette, was zu deren Spaltung führt.

Gezeigt wurden hier nun also drei verschiedene Optionen, mit denen es möglich ist, die bei niedrigen Laserfluenzen deponierten glatten PMMA-Schichten zu verändern und vor allem zu härten:

Durch die UV-Bestrahlung der Schichten wurde die Morphologie der Oberfläche nicht sichtlich beeinflusst. Ein Anstieg der mittleren Molmasse ließ erste Schlüsse auf eine Vernetzung zu. Durch den starken Abfall in der Intensität der $\pi_{\mathrm{C}=\mathrm{C}^{*}}^{*}$ Bindung lässt sich vermuten, dass diese Bindungen durch die UV-Strahlung gebrochen wurden und so zur Vernetzung beitragen konnten. Der Grund für den gleichzeitigen Intensi-

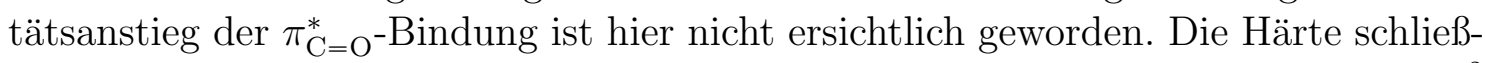
lich konnte abhängig von der Bestrahlungs- und Wartezeit auf über $250 \mathrm{~N} \mathrm{~mm}^{-2}$ erhöht werden. Die Aushärtung der bestrahlten Schichten dauert aber über mehrere Monate an, bis alle erzeugten Radikale einen neuen Bindungspartner gefunden haben.

Durch die Zugabe einer vernetzenden Komponente zum Targetmaterial wurde die Absorption im Target erhöht. Das hier verwendete Bisphenol A Dimethacrylat zeigte als reine Schicht zwar eine glatte Oberfläche, induzierte aber in den Mischschichten PMMA-Tröpfchen. Die mittlere Molmasse wurde durch die Zugabe des BisDMA leicht erhöht, ging aber bei einem zu großen Anteil wieder zurück. Auch der Anteil der nicht reagierten BisDMA-Moleküle konnte erhöht nachgewiesen werden. Mit zunehmendem Anteil an BisDMA konnte die Universalhärte der Schichten erhöht werden. Der maximale Härtewert wurde für eine reine BisDMA-Schicht mit $180 \mathrm{~N} \mathrm{~mm}^{-2}$ erreicht.

Die thermische Behandlung von PMMA bewirkt eine verstärkte Entnetzung der Schichten vom Siliziumsubstrat. Für ausreichend dicke Schichten, wie sie z. B. für die Untersuchungen der Universalhärte verwendet wurden, spielt dies allerdings im relevanten Temperaturbereich keine Rolle. Für die Härte konnte ein Anstieg oberhalb von $60^{\circ} \mathrm{C}$ festgestellt werden, der bis etwa $150{ }^{\circ} \mathrm{C}$ andauert und dann bei einer maximal erreichbaren Universalhärte von etwa $200 \mathrm{~N} \mathrm{~mm}^{-2}$ bleibt. Auch für sehr viel höhere Temperaturen ist dieser Wert noch messbar, solange es sich um eine „intakte“ Schicht handelt. Für degradierte Bereiche liegt die gemessene Universalhärte um einiges höher, allerdings sind diese Schichten aufgrund ihrer Oberflächenbeschaffenheit nicht weiter von Interesse.

Vergleichend lässt sich also feststellen, dass die UV-bestrahlten PMMA-Schichten die höchsten Härtewerte erreichen. Diese sind aber nicht sehr definiert einstellbar, da die bestrahlten Schichten über lange Zeiten nachhärten. Außerdem müssen hier Schichten für eine Härtung aus der PLD-Anlage ausgebaut werden. Mit Hilfe von BisDMA in den PMMA-Targets lässt sich zwar die Universalhärte etwas erhöhen, aber nicht in solchem Maße wie eben beschrieben und es entstehen Tröpfchen auf 
der Oberfläche. Die reinen BisDMA-Schichten sind allerdings härter als Schichten mit PMMA-Anteil und außerdem tröpfchenfrei. Aber auch diese reinen BisDMASchichten kommen im Härtevergleich nicht an die UV-bestrahlten Schichten heran. Die thermisch beeinflussten Schichten zeigen für dünnere Schichten eine starke Veränderung der Morphologie durch die entstehende Entnetzung. Sehr dicke Schichten sind aber bis mindestens $200^{\circ} \mathrm{C}$ weiterhin glatt und zeigen in diesem Temperaturbereich einen sehr gut definierten Härteanstieg bis etwa $200 \mathrm{~N} \mathrm{~mm}^{-2}$. Diese Werte bleiben auch über Wochen hinweg stabil.

Die im Rahmen dieser Arbeit beschriebenen Möglichkeiten, einen Härteanstieg der glatten PMMA-Schichten zu erzeugen, werden nun auch für die am Ende von Kapitel 4.5 erwähnten Schichtpakete erfolgreich angewendet und im Rahmen der Dissertation von SEYFFARTH näher untersucht. 


\section{Zusammenfassung}

Ziel dieser Arbeit war die Charakterisierung und Modifizierung glatter laserdeponierter Poly(methyl methacrylat)-Schichten. Grundlage hierfür waren die aus der vorangegangenen Diplomarbeit gewonnenen Kenntnisse zur gepulsten Laserdeposition von Polymeren vor allem in Hinblick auf den bedeutenden Parameter der dabei verwendeten Laserfluenz. Da es dort zum ersten Mal möglich war, glatte PMMASchichten mit der PLD herzustellen, sollte nun durch eine umfassende Charakterisierung dieser Schichten ein allgemeineres Verständnis über die zugrundeliegenden Prozesse der Ablation und damit der Deposition von Polymeren erarbeitet werden.

Dazu gehörten neben den detaillierten Untersuchungen des Inkubationsprozesses auch Morphologiebetrachtungen, chemische Analysen und die Untersuchung der mechanischen Eigenschaften. Ebenso interessant für weitere Anwendungen der Schichten waren die Untersuchungen zur thermischen Stabilität und zur Beeinflussung weiterer Eigenschaften durch thermische Behandlung. Anhand dieser Ergebnisse konnte die durch den Herstellungsprozess bedingte Zusammensetzung der deponierten Schichten besser verstanden werden. Vor allem aufgrund der Ergebnisse der mechanischen Messungen der glatten PMMA-Schichten wurden im weiteren Verlauf der Arbeit verschiedene Möglichkeiten der Schichthärtung untersucht. Die drei, hierfür ausgewählten Ansätze der Beeinflussung der mechanischen Eigenschaften - die UVBestrahlung der Schichten, die Beimischung einer vernetzenden Komponente zum Targetmaterial und die thermische Behandlung - wurden auch auf eine damit zusammenhängende Modifizierung der Morphologie und der chemischen Eigenschaften untersucht. In dieser Arbeit ist es mit allen drei Modifizierungsansätzen gelungen, die deponierten Schichten zu härten, womit praktische Anwendungen nun sehr gut möglich sind.

Die Ergebnisse dieser Arbeit zeigten, dass der Deposition von glatten PMMASchichten bei den hier verwendeten Laserfluenzen von nur etwa $125 \mathrm{~mJ} \mathrm{~cm}^{-2}$ - also leicht oberhalb der Depositionsschwelle des PMMA - ein ausgeprägter Inkubationsprozess voran geht. Während bei höheren Laserfluenzen die Inkubation schon während des ersten Pulses abgeschlossen wird und damit nicht von der Ablation des Materials unterschieden werden kann, dauert hier der Inkubationsprozess über 300 Pulse an. Dadurch ist die dazugehörige Vergilbung des PMMA eindeutig zu erkennen. Die daran anschließende Deposition produziert glatte Schichten, die in der Lage sind, anfängliche Rauigkeiten zu glätten. Diese glatten Schichten rauen auch mit steigender Schichtdicke nicht wieder auf und zeigen eine RMS-Rauigkeit von nur $0,3 \mathrm{~nm}$. Durch den Depositionspozess ergab sich eine Verringerung der $\mathrm{C}=\mathrm{O}$ - 
Doppelbindungen in den Schichten, was auf eine Abspaltung von ganzen oder Teilen von Estergruppen hinweist. Außerdem wurden die durch Inkubation entstandenen $\mathrm{C}=\mathrm{C}$-Doppelbindungen nachgewiesen. Durch diese Doppelbindungsbildung während des Inkubationsprozesses wurde die Absorption im Material erhöht. Zur Bildung neuer Bindungen ist eine Spaltung der Polymerketten erforderlich. Die glatten Schichten zeigten also eine starke Reduzierung der mittleren Molmasse mit Werten zwischen $2.200 \mathrm{~g} \mathrm{~mol}^{-1}$ und $2.900 \mathrm{~g} \mathrm{~mol}^{-1}$ mit einer Polydispersität $D$ von 2,3 bzw. 3,5. Dass die glatten Schichten aufgrund des Depositionsprozesses nicht vernetzt wurden, zeigte sich nicht nur durch die Möglichkeit, die Schichten für die SEC-Messungen in THF zu lösen. Die Schichten wiesen außerdem eine sehr geringe Universalhärte von etwa $3 \mathrm{~N} \mathrm{~mm}^{-2}$ auf. Aufgrund dieser geringen Härte, wurden in der Arbeit mehrere Möglichkeiten diskutiert, wie die Härte der Schichten erhöht werden könnte, um die praktische Anwendung der Schichten zu verbessern.

Für weitere Anwendungen ist auch die thermische Stabilität der PMMA-Schichten von großem Interesse. Durch die Reduzierung der mittleren Molmasse wurde eine Absenkung der Glasübergangstemperatur für die deponierten Schichten im Vergleich zum Ausgangsmaterial $\left(T_{g}=114^{\circ} \mathrm{C}\right)$ vermutet. Die tatsächlich gemessene Glasübergangstemperatur war aber mit $T_{g}=109^{\circ} \mathrm{C}$ kaum niedriger. Dies wurde unter anderem auf die Abspaltung der Seitenketten zurückgeführt. Dadurch wird hier die Glasübergangstemperatur umgekehrt zur bekannten Abnahme der Glasübergangstemperatur für höhere Poly(alkyl methacrylat)e (PAMA) beeinflusst, bei denen die Länge der Estergruppe anwächst. Außerdem wurde bei diesem Vergleich der gemessenen Glasübergangstemperaturen mit der Literatur nicht berücksichtigt, dass sich durch den Temperatureinfluss während der Messung von $T_{g}$ die mittlere Molmasse vergrößert. Damit würde der Messwert der Glasübergangstemperatur zu einer höheren Kettenlänge gehören und wäre auch wieder näher an der Literaturkurve der Glasübergangstemperaturen für die verschiedenen Kettenlängen von PMMA. Ebenso spielt die Vernetzung des Schichtmaterials eine wichtige Rolle, da mit höherem Vernetzungsgrad auch $T_{g}$ ansteigt. Bei einem sehr hohen Grad der Vernetzung findet sogar überhaupt kein Glasübergang mehr statt.

In den PMMA-Schichten lagen durch den Depositionsprozess auch abgespaltene Estergruppen oder Fragmente des PMMA vor. Dies konnte durch Massenspektrometrie nachgewiesen werden, da diese Moleküle bei Temperaturen oberhalb der Glasübergangstemperatur aufgrund der höheren Mobilität aus der Schicht dampfen können. Außerdem fanden bei diesen Temperaturen auch schon Spaltungen statt, durch die Quervernetzungen ermöglicht wurden. So machte sich bis $200^{\circ} \mathrm{C}$ ein Masseverlust von $40 \%$ bemerkbar, aber gleichzeitig wurde der eben erwähnte Anstieg der mittleren Molmasse auf $7.400 \mathrm{~g} \mathrm{~mol}^{-1}(D=9,5)$ verzeichnet. Der festgestellte zweistufige Massenverlust bei einer thermischen Auslagerung der PMMA-Schichten konnte durch die massenspektrometrische Analyse der Schichtfragmente als bimodale Verteilung in den Massenspektren verifiziert werden. Die Fragmente der ersten Stufe wurden hauptsächlich zwischen $130-150^{\circ} \mathrm{C}$ detektiert und wurden auf eine 
Aufspaltung des Monomers mit Wasserstoffabspaltung und auf eine Teilung der Estergruppe in Carbonylgruppen (CO) und Aldehyde (CHO) zurückgeführt. Für die Abspaltung von Estergruppen sprach ebenfalls ein Verlust in der mit NEXAFS gemessenen Intensität der $\pi_{\mathrm{C}=\mathrm{O}^{*}}^{*}$ Bindung. Der entsprechende Anstieg in der Intensität der $\pi_{\mathrm{C}=\mathrm{C}^{-}}^{*}$ Bindung wurde durch eine Umlagerung der frei gewordenen Bindungen nicht nur zu benachbarten Ketten (Quervernetzung) sondern auch zur eigenen Kette (Doppelbindungsbildung) beschrieben.

Die thermische Zersetzung des Materials wurde durch die zweite Stufe im Massenverlust der PMMA-Schicht zwischen $370-410{ }^{\circ} \mathrm{C}$ bzw. durch den hauptsächlichen Masseverlust des PMMA-Targets oberhalb von $330^{\circ} \mathrm{C}$ gezeigt. Für das deponierte Schichtmaterial ließen sich nun vermehrt kleinere Fragmente detektieren. Die mittlere Molmasse der Schichten verringerte sich bei diesen hohen Temperaturen wieder auf $2.200 \mathrm{~g} \mathrm{~mol}^{-1}(D=3,5)$.

Diese Modifizierung des PMMA durch thermischen Einfluss hatte auch Änderungen der Morphologie und vor allem der Härte der deponierten Schichten zur Folge. Diese Modifizierung der Schichten wurde in dieser Arbeit ebenso diskutiert wie die Modifizierung durch Bestrahlung der Schichten mit ultraviolettem Licht und durch die Beigabe eines vernetzenden Materials wie Bisphenol A Dimethacrylat (BisDMA) zum PMMA-Pulver vor der Targetpressung.

Die UV-Bestrahlung der Schichten hatte keinen Einfluss auf die Morphologie der Oberfläche, wobei aber nach $1 \mathrm{~h}$ Bestrahlzeit ein Anstieg der mittleren Molmasse auf $5.100 \mathrm{~g} \mathrm{~mol}^{-1}(D=5,0)$ erste Schlüsse auf eine Vernetzung zuließ. Durch den starken Abfall in der Intensität der $\pi_{\mathrm{C}=\mathrm{C}^{*}}^{*}$ Bindung lässt sich vermuten, dass diese Bindungen durch die UV-Strahlung gebrochen wurden und so zur Vernetzung beitragen konnten. Der Grund für den gleichzeitigen Intensitätsanstieg der $\pi_{\mathrm{C}=\mathrm{O}}^{*}$-Bindung ist hier nicht ersichtlich geworden. Abhängig von der Bestrahlungs- und Wartezeit konnte die Härte durch UV-Bestrahlung auf über $250 \mathrm{~N} \mathrm{~mm}^{-2}$ erhöht werden. Die erzeugten Radikale können aber sehr lange Zeit brauchen, bis sie einen neuen Bindungspartner gefunden haben. Dadurch können die bestrahlten Schichten über mehrere Monate nachhärten.

Durch die Zugabe einer vernetzenden Komponente zum Targetmaterial wurde die Absorption im Target erhöht. Das hierfür eingesetzte Bisphenol A Dimethacrylat zeigte als reine Schicht zwar eine glatte Oberfläche, induzierte aber in den Mischschichten PMMA-Tröpfchen. Die mittlere Molmasse wurde durch die steigende Vernetzung des Schichtmaterials durch die Zugabe des BisDMA zunächst leicht erhöht auf $4.400 \mathrm{~g} \mathrm{~mol}^{-1}(D=4,8)$, ging aber durch eine Übersättigung des PMMA durch einen zu großen kurzkettigen Vernetzeranteil wieder zurück auf $3.500 \mathrm{~g} \mathrm{~mol}^{-1}$ $(D=4,3)$. So konnte auch der Anteil der nicht reagierten BisDMA-Moleküle erhöht nachgewiesen werden. Die Universalhärte der Schichten stieg mit zunehmenden Vernetzungspunkten, also mit zunehmendem BisDMA-Anteil, wodurch sich der maximale Härtewert hier für eine reine BisDMA-Schicht mit $180 \mathrm{~N} \mathrm{~mm}^{-2}$ ergab.

Die zuvor ausführlich beschriebene thermische Behandlung von PMMA bewirkt 
eine verstärkte Entnetzung der Schichten vom Siliziumsubstrat. Für ausreichend dicke Schichten, wie sie z. B. für die Untersuchungen der Universalhärte verwendet wurden, spielte dies allerdings im relevanten Temperaturbereich keine Rolle. Für die Härtezunahme konnte ein definierter Anstieg oberhalb von $60^{\circ} \mathrm{C}$ festgestellt werden, der oberhalb von $150{ }^{\circ} \mathrm{C}$ endet und dann bei einer maximal erreichbaren Universalhärte von etwa $200 \mathrm{~N} \mathrm{~mm}^{-2}$ verbleibt. Auch für sehr viel höhere Temperaturen änderte sich diese Härte nicht mehr, sofern die Schicht noch nicht degradiert war. Für degradierte Bereiche lag die gemessene Universalhärte um einiges höher, wobei diese Schichten aufgrund ihrer Oberflächenbeschaffenheit nicht weiter von Interesse waren.

Im Vergleich dieser drei Methoden in Bezug auf die Schichthärtung erreichten die UV-bestrahlten PMMA-Schichten die höchsten Härtewerte. Da diese Schichten aber über lange Zeit nachhärten, können nur schwer definierte Härtewerte eingestellt werden. Zudem wurden die Schichten für diese Arbeit ex-situ, also außerhalb der PLDKammer, bestrahlt. Durch das Einbringen von BisDMA in die PMMA-Targets wurde zwar die Universalhärte der daraus deponierten Schichten etwas erhöht, die Werte kamen aber nicht an die Werte für die UV-bestrahlten Schichten heran. Außerdem entstanden Tröpfchen auf der Oberfläche. Die reinen BisDMA-Schichten waren dagegen tröpfchenfrei und härter als die Schichten mit PMMA-Anteil. Aber auch diese reinen BisDMA-Schichten kommen im Härtevergleich nicht an die UV-bestrahlten Schichten heran. Die in-situ thermisch beeinflussten Schichten waren bis mindestens $200{ }^{\circ} \mathrm{C}$ weiterhin glatt, sofern sie eine ausreichende Dicke aufwiesen, und zeigten in diesem Temperaturbereich einen sehr definierten Härteanstieg bis etwa $200 \mathrm{~N} \mathrm{~mm}^{-2}$. Im Vergleich zu den UV-bestrahlten Schichten blieben diese Werte auch über Wochen hinweg stabil.

Die Untersuchungen dieser Arbeit lieferten wichtige Eigenschaften - die sehr geringe Rauigkeit und die Möglichkeit der Härtung - für zukünftige Anwendungen der hier vorgestellten PMMA-Schichten. Eine erste Anwendung fanden die glatten PMMA-Schichten z. B. bereits im Rahmen der Dissertation von RöDER zur Glättung rauer Strukturen. Eine anderes Beispiel für die vielen Anwendungsmöglichkeit der hier diskutierten Schichten ist z. B. im Rahmen der Dissertation von SEYFFARTH die Verwendung für Polymer/Metall-Schichtpakete, zu denen in dieser Arbeit schon erste Versuche unternommen wurden.

Zusammenfassend kann also festgehalten werden, dass in dieser Arbeit glatte laserdeponierte PMMA-Schichten detailliert charakterisiert und erfolgreich modifiziert werden konnten. Alle drei Modifizierungsversuche - vor allem in Bezug auf die Härtung - waren erfolgreich. Dabei konnten die Schichten durch die UV-Bestrahlung am stärksten gehärtet werden, durch die thermische Behandlung lassen sich aber die Härtewerte sehr viel definierter einstellen. 


\section{Literaturverzeichnis}

[1] J. E. Andrew, P. E. Dyer, D. Forster, and P. H. Key, Direct etching of polymeric materials using a XeCl laser, Appl. Phys. Lett. 43 (1983), 717.

[2] C. A. Angell, Formation of Glasses from Liquids and Biopolymers, Science 267 (1995), 1924.

[3] N. Arnold and N. Bityurin, Model for laser-induced thermal degradation and ablation of polymers, Appl. Phys. A 68 (1999), 615.

[4] R. M. A. Azzam and N. M. Bashara, Ellipsometry and Polarized Light, Elsevier B. V., Amsterdam, The Netherlands, 1987.

[5] A. K. Baker and P. E. Dyer, Refractive-Index Modification of PolyMethylMethAcrylate) (PMMA) Thin Films by KrF-Laser Irradiation, Appl. Phys. A 57 (1993), 543 .

[6] H.-H. Behncke, Kraft-Eindringtiefen Verfahren: Wie vergleichbar sind Härtewerte?, VDI Berichte Nr. 1194 (1995), 33.

[7] G. B. Blanchet, Deposition of Poly(methyl methacrylate) Films by UV Laser Ablation, Makromolecules 28 (1995), 4603.

[8] G. B. Blanchet, P. Cotts, and Jr. C.R. Fincher, Incubation: Subthreshold ablation of poly-(methyl methacrylate) and the nature of the decomposition pathways, J. Appl. Phys. 88 (2000), 2975.

[9] M. Buback, H. Frauendorf, F. Günzler, and P. Vana, Electrospray ionization mass spectrometric end-group analysis of PMMA produced by radical polymerization using diacyl peroxide initiators, Polymer 48 (2007), 5590.

[10] M. Buback, P. Hesse, T. Junkers, and P. Vana, Determination of Addition and Fragmentation Rate Coefficients in RAFT Polymerization via Time-Resolved ESR Spectroscopy after Laser Pulse Initiation, Macromol. Rapid Commun. 27 (2006), 182.

[11] A. Chapiro, Radiation Chemistry of Polymeric Systems, pp. 352, 361 und 371, Wiley, New York, 1962. 
[12] H. Choi-Yim, D. Xu, and W. L. Johnson, Ni-based bulk metallic glass formation

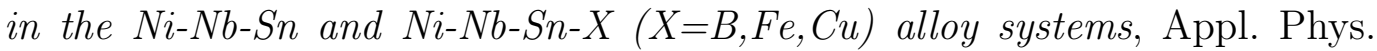
Lett. 82 (2003), 1030.

[13] S. Y. Chou, P. R. Krauss, and P. J. Renstrom, Imprint of sub-25 nm vias and trenches in polymers, Appl. Phys. Lett. 67 (1995), 3114.

[14] D. B. Chrisey and G. K. Hubler, Pulsed Laser Deposition of Thin Films, John Wiley \& Sons, Inc., New York, NY, 1994.

[15] D. B. Chrisey, A. Piqué, R. A. McGill, J. S. Horwitz, B. R. Ringeisen, D. M. Bubb, and P. K. Wu, Laser Deposition of Polymer and Biomaterial Films, Chem. Rev. 103 (2003), 553.

[16] J. Davenas, X. L. Xu, C. Khodr, M. Treilleux, and G. Steffan, A percolation approach to ion beam induced modifications of organic resists, Nucl. Instrum. Meth. Phys. Res. B 7/8 (1985), 513.

[17] G. M. Davis, M. C. Gower, C. Fotakis, T. Efthimiopoulos, and P. Argyrakis, Spectroscopic Studies of ArF Laser Photoablation of PMMA, Appl. Phys. A 36 (1985), 27.

[18] P. G. de Gennes, Reptation of a Polymer Chain in the Presence of Fixed Obstacles, J. Chem. Phys. 55 (1971), 572.

[19] J. Dechant, Ultrarotspektroskopische Untersuchungen an Polymeren, pp. 35, 319 und 368, Akademie-Verlag, Berlin, 1972.

[20] D. Dijkamp, T. Venkatesan, X. D. Wu, S. A. Shaheen, N. Jisrawi, Y. H. MinLee, W. L. Mclean, and M. Croft, Preparation of $Y$-Ba-Cu oxide superconductor thin films using pulsed laser evaporation from high- $T_{c}$ bulk material, Appl. Phys. Lett. 51 (1987), 619.

[21] E. Donth, M. Beiner, S. Reissig, J. Korus, F. Garwe, S. Vieweg, S. Kahle, E. Hempel, and K. Schröder, Fine Structure of the Main Transition in Amorphous Polymers: Entanglement Spacing and Characteristic Length of the Glass Transition. Discussion of Examples, Macromolecules 29 (1996), 6589.

[22] P. E. Dyer, Excimer laser polymer ablation: twenty years on, Appl. Phys. A 77 (2003), 167.

[23] R. Eason, Pulsed Laser Deposition of Thin Films, p. 220 ff., John Wiley \& Sons, Inc., Hoboken, New Jersey, 2007. 
[24] Christian Eberl, Härteänderung durch UV-Bestrahlung von laserdeponierten Poly(ethyl methacrylat)-Schichten, Bachelorarbeit, Institut für Materialphysik, Universität Göttingen, 2009.

[25] S. R. Elliot, Physics of amorphous materials, Longman Scientific \& Technical, Harlow, 1989.

[26] J. Faupel, C. Fuhse, A. Meschede, C. Herweg, H.-U. Krebs, and S. Vitta, Microstructure of pulsed laser deposited ceramic-metal and polymer-metal nanocomposited thin films, Appl. Phys. A 79 (2004), 1233.

[27] Jörg Faupel, Wachstum von metallischen Nanoclustern auf Polymeroberflächen, Dissertation, Institut für Materialphysik, Universität Göttingen, 2005.

[28] W. W. Flack, D. S. Soong, A. T. Bell, and D. W. Hess, A mathematical model for spin coating of polymer resists, J. Appl. Phys. 56 (1984), 1199.

[29] D. S. Fryer, P. F. Nealey, and J. J. de Pablo, Thermal Probe Measurements of the Glass Transition Temperature for Ultrathin Polymer Films as a Function of Thickness, Macromolecules 33 (2000), 6439.

[30] D. S. Fryer, R. D. Peters, E. J. Kim, J. E. Tomaszewski, J. J. de Pablo, P. F. Nealey, C. C. White, and W.-L. Wu, Dependence of the Glass Transition Temperature of Polymer Films on Interfacial Energy and Thickness, Macromolecules 34 (2001), 5627.

[31] B. Fuchs, F. Schlenkrich, S. Seyffarth, A. Meschede, R. Rotzoll, P. Vana, P. Großmann, K. Mann, and H.-U. Krebs, Hardening of smooth pulsed laser deposited PMMA films by heating, Appl. Phys. A 98 (2010), 711.

[32] H. Futurani, H. Fukumura, H. Masuhara, T. Lippert, and A. Yabe, LaserInduced Decomposition and Ablation Dynamics Studied by Nanosecond Interferometry. 1. A Triazenopolymer Film, J. Phys. Chem. A 101 (1997), 5742.

[33] Sebastian Fähler, Laserablation und Deposition von Metallen: Untersuchungen zum Prozess, Diplomarbeit, Institut für Materialphysik, Universität Göttingen, 1994.

[34] Sebastian Fähler, Der Einfluss der kinetischen Energie der Ionen auf das Wachstum laserdeponierter metallischer Schichten und Schichtpakete: Untersuchungen mit Flugzeitmessungen und RHEED/THEED, Dissertation, Institut für Materialphysik, Universität Göttingen, 1998.

[35] B. J. Garrison and R. Srinivasan, Microscopic model for the ablative photodecomposition of polymers by far-ultraviolet radiation (193 nm), J. Appl. Phys. 44 (1984), 849. 
[36] R. M. Gilgenbach and P. L. G. Ventzek, Dynamics of excimer laser-ablated aluminum neutral atom plume measured by dye laser resonance absorption photography, Appl. Phys. Lett. 58 (1991), 1597.

[37] J. L. Gómez Ribelles, M. Monleón Pradas, A. Vidaurre Garayo, F. Romero Colomer, J. Más Estellés, and J. M. Meseguer Dueñas, Structural Relaxation of Glass-Forming Polymers Based on an Equation for Configurational Entropy. 2. Structural Relaxation in Polymethacrylates, Macromolecules 28 (1995), 5878 .

[38] J. G. Goodberlet, Patterning 100 nm features using deep-ultraviolet contact photolithography, Appl. Phys. Lett. 76 (2000), 667.

[39] A. L. Greer, Metallic Glasses, Science 267 (1995), 1947.

[40] P. Grünberg, Viskoelastische Eigenschaften, 22. IFF-Ferienkurs: Physik der Polymere (R. Hölzle, ed.), Forschungszentrum Jülich GmbH, Jülich, 1991, p. $14.1 \mathrm{ff}$.

[41] Amitava Gupta, Ranty Liang, Fun Dow Tsay, and Jovan Moacanin, Characterization of a Dissociative Excited State in the Solid State: Photochemistry of Poly(methyl methacrylate). Photochemical Processes in Polymeric Systems., Makromolecules 13 (1980), 1696.

[42] T. M. Hall, A. Wagner, and L. F. Thompson, Ion beam exposure characteristics of resists, J. Vac. Sci. Technol. 16 (1979), 1889.

[43] T. M. Hall, A. Wagner, and L. F. Thompson, Ion beam exposure characteristics of resists: Experimental results, J. Appl. Phys. 53 (1982), 3997.

[44] S. G. Hansen and T. E. Robitaille, Arrival time measurements of films formed by pulsed laser evaporation of polycarbonate and selenium, J. Appl. Phys. 64 (1988), 2122.

[45] S. G. Hansen and T. E. Robitaille, Formation of polymer films by pulsed laser evaporation, Appl. Phys. Lett. 52 (1988), 81.

[46] J. Heitz, E. Arenholz, T. Kefer, D. Bäuerle, H. Hibst, and A. Hagemeyer, Enhanced Adhesion of Metal Films on PET after UV-Laser Treatment, Appl. Phys. A 55 (1992), 391.

[47] A. C. F. Hoole, M. E. Welland, and A. N. Broers, Negative PMMA as a high-resolution resist - the limits and possibilities, Semicond. Sci. Technol. 12 (1997), 1166. 
[48] R. Hölzle (Hrsg.), 22. IFF-Ferienkurs: Physik der Polymere, Forschungszentrum Jülich GmbH, Jülich, 1991.

[49] H. H. G. Jellinek and Ming Dean Luh, Thermal Degradation of Polymethylmethacrylate: Energies of Activation, Makromol. Chemie 115 (1968), 89.

[50] R. M. Jones, Mechanics of Composite Materials, Taylor and Francis, Philadelphia, 1999.

[51] R. Kelly, A. Miotello, B. Braran, A. Gupta, and K. Casey, Primary and secondary mechanisms in laser-pulse sputtering, Nucl. Instr. Meth. B 65 (1992), 187.

[52] H.-U. Krebs, Characteristic properties of laser-deposited metallic systems, J. Non-Equilibrium Proc. 10 (1997), 3.

[53] H.-U. Krebs and O. Bremert, Pulsed laser deposition of thin metallic alloys, Appl. Phys. Lett. 62 (1993), 2341.

[54] H.-U. Krebs, S. Fähler, and O. Bremert, Laser deposition of metallic alloys and multilayers, Appl. Surf. Sci. 86 (1995), 86.

[55] S.-W. Kuo, H.-C. Kao, and F.-C. Chang, Thermal behavior and specific interaction in high glass transition temperature PMMA copolymer, Polymer 44 (2003), 6873.

[56] S. Küper, S. Modaressi, and M. Stuke, Photofragmentation Pathways of a PMMA Model Compound under UV Excimer Laser Ablation Conditions, J. Phys. Chem. 94 (1990), 7514.

[57] S. Küper and M. Stuke, Femtosecond uv Excimer Laser Ablation, Appl. Phys. B 44 (1987), 199.

[58] S. Küper and M. Stuke, UV-Excimer-Laser Ablation of Polymethylmethacrylate at $248 \mathrm{~nm}$ : Characterization of Incubation Sites with Fourier Transform IRand UV-Spectroscopy, Appl. Phys. A 49 (1989), 211.

[59] R. Larciprete and M. Stuke, Direct Observation of Excimer-Laser Photoablation Products from Polymers by Picosecond-UV-Laser Mass Spectroscopy, Appl. Phys. B 42 (1987), 181.

[60] S. Lazare and V. Granier, Excimer laser light induced ablation and reactions at polymer surfaces as measured with a quartz-crystal microbalance, J. Appl. Phys. 63 (1988), 2110. 
[61] S. Lazare, J. Lopez, and F. Weisbuch, High-aspect-ratio microdrilling in polymeric materials with intense KrF laser radiation, Appl. Phys. A 69 (1999), S1.

[62] E. H. Lee, Y. Lee, W. C. Oliver, and L. K. Mansur, Hardness measurements of $\mathrm{Ar}^{+}$-beam treated polyimide by depth-sensing ultra low load indentation, J. Mater. Res. 8 (1993), 377.

[63] E. H. Lee, G. R. Rao, and L. K. Mansur, Hardness Enhancement and Crosslinking Mechanisms in Polystyrene Irradiated with High Energy Ion-Beams, Mat. Sci. Forum 248-249 (1997), 135.

[64] E. H. Lee, G. R. Rao, and L. K. Mansur, LET effect on cross-linking and scission mechanisms of PMMA during irradiation, Rad. Phys. Chem. 55 (1999), 293.

[65] D. Li, Y. Chen, Y.-W. Chung, and F. L. Freire, Metrology of 1-10 nm thick $C N_{x}$ films: Thickness, density, and surface roughness measurements, J. Vac. Sci. Tech. A 21 (2003), L 19.

[66] T. Lippert, Interaction of Photons with Polymers: From Surface Modification to Ablation, Plasma Process. Polym. 2 (2005), 525.

[67] T. Lippert and J. T. Dickinson, Chemical and Spectroscopic Aspects of Polymer Ablation: Special Features and Novel Directions, Chem. Rev. 103 (2003), 453.

[68] T. Lippert, R. L. Webb, S. C. Langford, and J. T. Dickinson, Dopant induced ablation of poly(methyl methacrylate) at 308 nm, J. Appl. Phys. 85 (1999), 1838.

[69] B. Lösekrug, A. Meschede, and H.-U. Krebs, Pulsed laser deposition of smooth poly(methyl methacrylate) films at 248 nm, Appl. Surf. Sci. 254 (2007), 1312.

[70] Britta Lena Lösekrug, Energiedichteabhängigkeit der Eigenschaften laserdeponierter Poly(methyl methacrylat)-Schichten und -Kompositmaterialien, Diplomarbeit, Institut für Materialphysik, Universität Göttingen, 2006.

[71] Lewis E. Manring, Thermal Degradation of Poly(methyl methacrylate). 4. Random Side-Group Scission, Macromolecules 24 (1991), 3304.

[72] Andreas Meschede, Wachstum und Eigenschaften laserdeponierter $\mathrm{MgO} / \mathrm{Fe}$ und Ti-Sc/Fe-Cr-Schichtsysteme, Diplomarbeit, Institut für Materialphysik, Universität Göttingen, 2004. 
[73] S. C. K. Misra, M. K. Ram, S. S. Pandey, B. D. Malhotra, and Subhas Chandra, Vacuum-deposited metal/polyaniline Schottky device, Appl. Phys. Lett. 61 (1992), 1219.

[74] G. Moad, E. Rizzardo, and S. H. Thang, Living Radical Polymerization by the RAFT process, Aust. J. Chem. 58 (2005), 379.

[75] C. E. Mortimer and U. Müller, Chemie: Das Basiswissen der Chemie, p. 58, Georg Thieme Verlag, Stuttgard, New York, 2001.

[76] C. T. Moynihan, Correlation between the Width of the Glass Transition Region and the Temperature Dependence of the Viscosity of High-Tg Glasses, J. Am. Ceram. Soc. 76 (1993), 1081.

[77] K. O'Driscoll and R. A. Sanayeu, Chain-Length Dependence of the Glass Transition Temperature, Macromolecules 24 (1991), 4479.

[78] C. Peth, F. Barkusky, and K. Mann, Near-edge x-ray absorption fine structure measurements using a laboratory-scale XUVl source, J. Phys. D: Appl. Phys. 41 (2008), 105202.

[79] G. H. Pettit and R. Sauerbrey, Pulsed Ultraviolet Laser Ablation, Appl. Phys. A 56 (1993), 51.

[80] D. Pham, L. Tonge, J. Cao, J. Wright, M. Papiernik, E. Harvey, and D. Nicolau, Effects of polymer properties on laser ablation behaviour, Smart Mater. Struct. 11 (2002), 668.

[81] J. F. Rabek, Photodegradation of Polymers, Springer Verlag, Berlin, Heidelberg, New York, 1996.

[82] N. Rehse, C. Wang, M. Hund, M. Geoghegan, R. Magerle, and G. Krausch, Stability of thin polymer films on a corrugated substrate, Eur. Phys. J. E 4 (2001), 69 .

[83] G. Reiter, Dewetting of Thin Polymer Films, Phys. Rev. Lett. 68 (1992), 75.

[84] Johanna Röder, Analyse der Glättung rauer Oberflächen durch Dünnschichtdeposition, Dissertation, Institut für Materialphysik, Universität Göttingen, 2009 .

[85] S. Sadki, Ph. Schottland, N. Brodie, and G. Sabouraud, The mechanisms of pyrrole electropolymerization, Chem. Soc. Rev. 29 (2000), 283.

[86] T. Scharf, J. Faupel, K. Sturm, and H.-U. Krebs, Intrinsic stress evolution in laser deposited thin films, J. Appl. Phys. 94 (2003), 4273. 
[87] Thorsten Scharf, Depositionsmechanismen, Struktur und mechanische Eigenschaften laserdeponierter Poly(methyl methacrylat)-Filme, Dissertation, Institut für Materialphysik, Universität Göttingen, 2006.

[88] S. Schiller, J. Hu, A. T. A. Jenkins, R. B. Timmons, F. S. Sanchez-Estrada, W. Knoll, and R. Förch, Chemical Structure and Properties of PlasmaPolymerized Maleic Anhydride Films, Chem. Mater. 14 (2002), 235.

[89] R. Seemann, S. Herminghaus, C. Neto, S. Schlagowski, D. Podzimek, R. Konrad, H. Mantz, and K. Jacobs, Dynamics and structure formation in thin polymer melt films, J. Phys.: Condens. Matter 17 (2005), S267.

[90] R. K. Singh, D. Bhattacharya, and J. Narayan, Subsurface heating effects during pulsed laser evaporation of materials, Appl. Phys. Lett. 57 (1990), 2022.

[91] H. M. Smith and A. F. Turner, Vacuum deposited thin films using a ruby laser, Appl. Opt. 4 (1965), 147.

[92] R. Srinivasan, Ablation of polymethyl methacrylate films by pulsed (ns) ultraviolet and infrared $(9.17 \mu \mathrm{m})$ lasers: A comparative study by ultrafast imaging, Appl. Phys. Lett. 73 (1993), 2743.

[93] R. Srinivasan and B. Braren, Ultraviolet Laser Ablation and Etching of Polymethyl Methacrylate Sensitized with an Organic Dopand, Appl. Phys. A 45 (1988), 289.

[94] R. Srinivasan, B. Braren, and K. G. Casey, Nature of "incubation pulses" in the ultraviolet laser ablation of polymethyl methacrylate, J. Appl. Phys. 68 (1990), 1842.

[95] R. Srinivasan, B. Braren, K. G. Casey, and M. Yeh, Ultrafast imaging of ultraviolet laser ablation and etching of polymethylmethacrylate, Appl. Phys. Lett. 55 (1989), 2790.

[96] R. Srinivasan, B. Braren, R. W. Dreyfus, L. Hadel, and D. E. Seeger, Mechanism of the ultraviolet laser ablation of polymethyl methacrylate at 193 and 248 nm: laser-induced fluorescence analysis, chemical analysis, and doping studies, J. Opt. Soc. Am. B 3 (1986), 785.

[97] R. Srinivasan and V. Mayne-Banton, Self-developing photoetching of poly(ethylene terephtalate) films by far-ultraviolet excimer laser radiation, J. Appl. Phys. 41 (1982), 576.

[98] Kai Sturm, Einfluß einer Argon-Atmosphäre auf die gepulste Laserdeposition von Metallschichten, Dissertation, Institut für Materialphysik, Universität Göttingen, 2000. 
[99] J. Stöhr, NEXAFS Spectroscopy, p. 208, Springer Verlag, Berlin, Heidelberg, New York, 1992.

[100] E. Sutcliffe and R. Srinivasan, Dynamics of UV laser ablation of organic polymer surfaces, J. Appl. Phys. 60 (1986), 3315.

[101] E. Süske, T. Scharf, H.-U. Krebs, E. Panchenko, T. Junkers, M. Egorov, M. Buback, and H. Kijewski, Tuning of cross-linking and mechanical properties of laser-deposited poly(methyl methacrylate) films, J. Appl. Phys. 97 (2005), 063501.

[102] E. Süske, T. Scharf, P. Schaaf, E. Panchenko, D. Nelke, M. Buback, H. Kijewski, and H.-U. Krebs, Variation of the mechanical properties of pulsed laser deposited pmma films during annealing, Appl. Phys. A 79 (2004), 1295.

[103] Erik Süske, Laserdeposition von Polymer-Metall-Schichten, Diplomarbeit, Institut für Materialphysik, Universität Göttingen, 2001.

[104] Erik Süske, Charakteristische Eigenschaften laserdeponierter Poly(methylmethacrylat)-Filme, Dissertation, Institut für Materialphysik, Universität Göttingen, 2005.

[105] A. Todd, The Mechanisms of Radiation-Induced Changes in Vinyl Polymers, J. Polym. Sci. 42 (1960), 223.

[106] Y. Tsuboi, N. Adachi, E. Yamamoto, and A. Itaya, Pulsed Laser Deposition of Poly(tetrafluoroethylene), Poly(methylmethacrylate) and Polycarbonate Utilizing Anthracene-Photosensitized Ablation, Jpn. J. Appl. Phys.. 41 (2002), 885 .

[107] T. Venkatesan, D. Edelson, and W. L. Brown, Pulsed ion beam technique for measuring diffusion coefficient of a slow diffusant in polymers, Appl. Phys. Lett. 43 (1983), 364.

[108] R. Viallard, M. Magat, and A. Chapiro (Ed.), Radiation Chemistry of Polymeric Systems, p. 103, Wiley, New York, 1962.

[109] D. L. Windt, IMD-Software for modelling the optical properties of multilayer films, Computers in Physics 12 (1998), 360.

[110] B. Wunderlich, Motion in Polyethylene. III. The Amorphous Polymer, J. Chem. Phys. 37 (1962), 2429.

[111] I. Zailer, J. E. F. Frost, V. Chabasseur-Molyneux, C. J. B. Ford, and M. Pepper, Crosslinked PMMA as a high-resolution negative resist for electron beam lithography and applications for physics of low-dimensional structures, Semicond. Sci. Technol. 11 (1996), 1235. 


\section{Danksagungen}

An dieser Stelle ist es nun an der Zeit, den vielen Menschen zu danken, die auf die eine oder andere Weise zum Gelingen dieser Arbeit beigetragen haben. So gilt der erste Dank Prof. Uli Krebs, der mir diese Arbeit erst ermöglicht hat und der schon an das Gute in meinen Ergebnissen geglaubt hat, während ich sie noch skeptisch betrachtete. Er hat mir mit diesem Thema und mit dem damit verbundenen Stipendium des Graduiertenkollegs 782 die Möglichkeit gegeben, eine enge Verbindung zwischen den Fakultäten für Physik und Chemie zu nutzen.

Deshalb geht ein weiterer Dank auch an Prof. Michael Buback, der mich die ganze Promotionszeit hindurch als Zweitbetreuer sowohl im Graduiertenkolleg als auch im Rahmen des Promotionskollegs GAUSS begleitet hat. Außerdem gilt ihm mein Dank für die Übernahme des Korreferats.

Ein gebündelter Dank geht vor allem an die PLD-Gruppe, die vielleicht doch nicht ganz zu Unrecht die „Kuschelgruppe“ genannt wurde. Man fühlte sich bei den unzähligen Diskussionen über die Physik bei einem Kaffee ebenso gut aufgehoben wie bei den Pausen zu viert, um den Kopf von den vielen Diskussionen wieder frei zu bekommen. Also Danke an jeden Einzelnen für die schöne Zeit:

An erster Stelle möchte ich dabei vor allem Andreas Meschede danken, der es so viele Jahre mit mir in einem Büro ausgehalten hat und der mit mir Höhen (Kaffee) und Tiefen (Raketen) des Büroalltags durchlebte und mit mir die Polymerphysik durchdiskutierte. Als Altdoktorand hatte er immer einen (guten) Ratschlag parat. Ich werde die halben Kaffees vermissen! Danke an Conni Mewes, die immer ein offenes Ohr für mich hatte und die einfach unser Mädchen für alles war von technischer Hilfe in sämtlichen Laboren über Bestellungen und dem damit verbundenen Verwaltungsaufwand bis hin zur Kummerkastentante.

Weiterhin in der PLD-Gruppe möchte ich Erik Süske und Thorsten Scharf für alles danken, was ich am Anfang über die Laserdeposition von PMMA wissen musste und an Frank Barkusky, der durch seine PMMA-Experimente im LaserLab immer ein anderes Licht auf die Sicht der Dinge geben konnte. Danke an Johanna Röder, dafür dass sie als erste meine Ergebnisse zu etwas gebrauchen konnte und vor allem für das unglaubliche Durchhaltevermögen als Lektorin dieser Arbeit. Bei Tobias Liese spreche ich wohl im Namen der ganzen Gruppe, wenn ich ihm für die ewig andauernde Arbeit an unserem Laserprogramm danke und möchte ihm gleichzeitig für die nächsten Jahre viel Spaß als Altdoktorand wünschen. Danke an Susanne Seyffarth für ihre immer gute Laune und für die schöne Zusammenarbeit im Labor, im Graduiertenkolleg, im Praktikum etc. Ich habe mir gerne mit ihr die Kammer 
geteilt! Danke an Felix Schlenkrich für die Hilfe mit NEXAFS und für das ständige Normieren. Danke an die neuesten Mitglieder der Gruppe Sarah Hoffmann und Benedikt Ernst, dafür dass sie einem zeigen, dass es nicht schlimm ist, sich auch mal ein blaues Auge zu holen und dass die Gruppe auch lange nach einem noch an den gleichen Herausforderungen weiterarbeiten wird. Danke an meinen ersten und einzigen Bachelor Christian Eberl, der mir gezeigt hat, wieviel Spaß es macht, eine Bachelorarbeit zu betreuen und das mein verwendetes Polymer doch gut zu handhaben ist.

Außerdem gehört ein Dankeschön Michael Malchow, der zu den hilfsbereitesten Menschen gehört, die ich kenne und ohne den unser 2. Stock nicht derselbe wäre. Ebenso danken möchte ich Dieter Plischke für sein großes Wissen am REM, Matthias Büchsenschütz-Göbeler für die Bilder am FIB und allen Mitgliedern des Instituts für ihre technische Hilfe und Unterstützung.

Im I. Physikalischen Institut geht mein Dank an Prof. Konrad Samwer und die Glasgruppe für die Gelegenheit an vielen Seminaren teilnehmen zu dürfen und für die damit verbundenen Diskussionen und die Hilfe. Ebenso ein Dank an Katrin Gehrke für die STA-Messungen.

In der Physikalischen Chemie geht mein Dank neben Herrn Suhm für die tolle Organisation des Graduiertenkollegs an Robert Rotzoll für die gute Zusammenarbeit, die SEC-Messungen und die Projektwoche und an Prof. Philipp Vana für die Diskussionen und die vielen guten Tipps.

Im LaserLab geht mein Dank an Peter Großmann und Klaus Mann, für die Möglichkeit NEXAFS-Messungen an den PMMA-Proben machen zu können.

An dieser Stelle möchte ich nun noch meinen Freunden (in Göttingen) danken, die mich zum größten Teil seit dem ersten Semester durch das Studium begleitet haben und die einem gezeigt haben, dass man zusammen immer stärker ist als allein. Ohne sie wäre die Zeit in Göttingen nicht so schön gewesen und so mancher Abend nicht so witzig.

Ein großes Dankeschön an meine Familie, die mich in allen Lebenslagen unterstützt und immer an mich geglaubt hat. Und danke an meine Schwiegerfamilie, dass sie mir die Daumen gedrückt hat, damit ich die erste Dr. Fuchs werde.

Der innigste und allergrößte Dank aber geht an meinen Mann und persönlichen Admin Sebastian. Ohne ihn wäre mein ganzes Studium und damit diese Doktorarbeit bestimmt ganz anders verlaufen. Mit ihm habe ich unzählige Herausforderungen gemeistert und ohne ihn hätte ich die eine oder andere Aufgabe vielleicht nie bewältigt. Danke für die viele Hilfe. Danke für die Rückendeckung. Danke für alles! 


\section{Lebenslauf}

\section{Persönliche Daten}

Name: Britta Lena Fuchs, geb. Lösekrug

Geburtsdaten: $\quad$ 27.07.1982 in Peine

Staatsangehörigkeit: deutsch

\section{Schulbildung}

1988-1990 Grundschule Woltwiesche, Landkreis Peine

1990-1992 Grundschule Lüchow, Landkreis Lüchow-Dannenberg

1992 Orientierungsstufe Lüchow, Landkreis Lüchow-Dannenberg

1993-1994 Orientierungsstufe Gartow, Landkreis Lüchow-Dannenberg

1994-2001 Gymnasium Lüchow, Landkreis Lüchow-Dannenberg

22.06.2001 Abitur

\section{Studium}

2001-2003 Grundstudium der Physik an der Georg-August-Universität Göttingen

17.07.2003 Vordiplom in Physik

2003-2007 Hauptstudium der Physik an der Georg-August-Universität Göttingen

2005-2007 Diplomarbeit bei Prof. Dr. H.-U. Krebs am Institut für Materialphysik, Titel der Arbeit: Energiedichteabhängigkeit der Eigenschaften laserdeponierter Poly(methyl methacrylat)-Schichten und-Kompositmaterialien

26.01.2007 Diplom in Physik

seit 2007 Aufbaustudium Promotion 